\title{
MAKING THE TRANSITION TO INSULIN THERAPY: THE EXPERIENCES OF SAMOAN PEOPLE WITH TYPE 2 DIABETES IN NEW ZEALAND.
}

By

Sera Tapu-Ta'ala

A thesis submitted to the Victoria University of Wellington

in partial fulfilment of the

requirements for the degree of

Master of Nursing (Clinical)

Victoria University of Wellington

2011 


\begin{abstract}
Background

Pacific people are dying younger compared to other New Zealanders because of complications resulting from uncontrolled type 2 diabetes mellitus. Good diabetes control is achievable with early use of insulin because of its effectiveness, and proven long term benefits to quality of life. An understanding of how Samoan people with type 2 diabetes make their transition to insulin therapy will assist in understanding how insulin is perceived, which will inform health care professionals in their work with those diagnosed with diabetes.
\end{abstract}

\title{
Aim of Research
}

The aim of the research is to explore and describe how Samoan people with type 2 diabetes in New Zealand made the transition to insulin therapy for better glycaemic control.

\section{Design}

The Fonofale Model was used as the theoretical framework, from which to understand Samoan peoples' experiences. This research used a qualitative descriptive methodology. In-depth interviews were used to gather the stories of four Samoan participants over the age of 18 years diagnosed with type 2 diabetes. The data was analysed using thematic analysis.

\section{Findings}

Three major themes emerged from the analysis of the participants' stories. These were: living with diabetes, making the transition to insulin therapy and realisation. The findings led to the creation of the Ia Malu model, which describes the experiences of the participants in this study.

\section{Conclusion}

This study confirmed that there are immense challenges and struggles encountered by people with diabetes. Their adjustment to the illness as well as making the transition to using insulin takes time. As a result of this, it is fundamental for nurses/health care 
professionals to understand that this is the reality for these people, and they must therefore provide time for people to adjust.

Keywords: $\quad$ Type 2 diabetes, Samoan people, transition to insulin therapy, qualitative descriptive methodology 


\section{Acknowledgements}

I am deeply indebted to the following people and would like to acknowledge their contribution to this thesis:

I would like to acknowledge Health Research Council for funding this study.

To the Participants: I am privileged for your time and feel very humble for the gifts you have given me through your stories. This thesis would not be written if it were not for you and your stories. Fa'afetai tele lava mo le 'oa ua outou fa'asoa mai ua mafai ai ona fa'a'oloaina lenei sa'ili'iliga, auā le nofo leleia o tagata Samoa, atoa ma tagata Pasefika uma o lo'o ua 'apo'ia e le ma'i suka i Niu Sila nei.

To my Supervisors: Words fail to express how much I appreciate each of you and the determination you each gave me to keep going. To Dr Karen McBride-Henry; thank you for believing in me and for helping me complete this thesis. Thank you so much for your constant reassurance when I doubted myself and for your understanding when I needed it. To Dr Cheryle Moss who began this journey with me; thank you so much for the insights and the courage you instilled in me so I could keep going with the research topic. Thank you for your knowledge during our early discussions of my research ideas. To Dr Margaret Martin who worked with me; thank you for your kind words when things were tough and for your time invested in me. I would also like to acknowledge Dr Kathy Nelson for her constructive input in the final phase of the thesis. I am sincerely grateful for all of your encouragement and tireless support.

To Reverend Nove Vaila'au: I am so grateful for your contribution, commitment and for sharing your wisdom through mentoring me while writing this thesis. Fa'afetai tele lava mo le tapasāina o lenei folauga faigata, auā e le'i faigofie mo a'u. 
To Fuimaono Karl Pulotu-Endermann: I would like to acknowledge you for trusting me to work with the Fonofale Model. I sincerely appreciate your work for Pacific people. Malo lava le fai o le faiva!

To the Pacific Providers: I am grateful for your endorsement of this thesis when I visited you about it. Ia manūteleina la outou galuega i le tautuaina o o tatou tagata Pasefika.

To UNESCO, Apia, Samoa: Thank you for permitting me to use one of your pictures of the fale Samoa for my model. Fa'afetai tele lava ona o le taliaina o le fa'atala'u'ula atu.

To Dr Robert Smith: Thank you for helping me see the value in researching the stories of Samoan participants, and for the time you gave to hear my research ideas.

To Ula Fatialofa and Elaine Ete-Rasch: Thank you for your presence when I needed to discuss my thesis progress. I am indebted to your friendship.

To Janet Dunbar: Thank you very much for your time given to proof read this thesis. I am grateful for your support and help.

To ALL my colleagues: Thank you so much for the words of encouragement that kept me going through this life changing experience. Thank you for your commitment in your work with people with diabetes. I do hope this piece of work is of benefits to you.

To my Employer: I am appreciative of all the support I received while working on this thesis. Thank you very much.

To all my Families and Friends: I acknowledge you all for keeping me sane when things seemed impossible. Thank you all for your prayers and thoughts. 
To my father Segi Ualesi Tapu: I am so thankful that you shared with me your wisdom on the uniqueness of the Fale Samoa, and for making me realise again the specialness about it. Fa'afetai tele tamā i lou tufa mai.

To my brother Pene-Huro Tapu: Thank you so much for sharing your gift as an artist to help me create the illustration for my health model. Fa'afetai tele lava mo lau fesoasoani.

And last but not least, to my beloved children (Raene, Kennyth \& Jaxsen Ualesi) and husband (Sene Ta'ala), and my dear parents (Segi Ualesi \& Malelega Tapu). I am so grateful for all the sacrifices you each made for me so I could keep writing to finally complete the thesis. I thank God everyday that you are my family. Oute fa'afetai tele atu mai i le ta'ele o lo'u nei fatu mo le tapua'iga, auā ua i'u ma le manuia lenei fa'amoemoe, a e ia viia ai pea le Atua.

O le mana'o ua taunu'u, o le la'au o le ola lea. (Proverbs: 13, v 12)

Fa'afetai tele lava. 


\section{Table of Contents}

Abstract $\quad$ ii

Acknowledgement iv

Table of Contents vii

$\begin{array}{ll}\text { Chapter One: } & \text { Introduction to the Research }\end{array}$

Research Question, Aim and Objectives 1

What is diabetes? 2

Type 1 diabetes 3

Type 2 diabetes 4

Trajectory of Type 2 diabetes 4

My clinical background $\quad 5$

Profiling Pacific people 5

$\begin{array}{ll}\text { Why this study is important } & 6\end{array}$

$\begin{array}{ll}\text { Background to the study } & 7\end{array}$

Theoretical Framework $\quad 9$

$\begin{array}{ll}\text { Introduction to Methodology } & 11\end{array}$

$\begin{array}{ll}\text { Document overview } & 11\end{array}$

$\begin{array}{ll}\text { Summary } & 12\end{array}$

$\begin{array}{lll}\text { Chapter Two: } & \text { The experience with Type } 2 \text { diabetes } & 13\end{array}$

$\begin{array}{ll}\text { Introduction } & 13\end{array}$

Table 1: Summary for search strategy 14

Section 1: $\quad$ The transitional journey to insulin 14

Section 2: $\quad$ Implications of Type 2 diabetes for Pacific people 17

Health of Pacific people $\quad 17$

$\begin{array}{lll}\text { Section 3: } & \text { Samoan people } & 18\end{array}$

Samoan people in New Zealand $\quad 18$

$\begin{array}{ll}\text { Samoan culture } & 19\end{array}$

$\begin{array}{ll}\text { Aiga } & 19\end{array}$ 
Section 4: The value of cultural differences and meaning for health 20

Health beliefs 20

$\begin{array}{ll}\text { Acculturation } & 21\end{array}$

Appreciating cultural differences $\quad 22$

What are the Pacific people saying? 23

The Fonofale Model 25

$\begin{array}{ll}\text { Summary } & 29\end{array}$

$\begin{array}{lll}\text { Chapter Three: } & \text { Research Methodology } & 30\end{array}$

Introduction $\quad 30$

Aim and Objectives of the Research 30

$\begin{array}{ll}\text { Research approach } & 31\end{array}$

Examples of Qualitative approach in Descriptive research 32

$\begin{array}{ll}\text { Research design } & 34\end{array}$

Overall feature of the study design $\quad 34$

Why this research is important? 35

Consultation 36

Treaty of Waitangi 36

$\begin{array}{ll}\text { Inclusion criteria for recruitment } & 37\end{array}$

$\begin{array}{ll}\text { Recruitment process } & 37\end{array}$

Data collection, sources and methods 38

$\begin{array}{ll}\text { Ethics } & 40\end{array}$

$\begin{array}{ll}\text { Data analysis } & 41\end{array}$

Consideration of rigour 42

Table 1: Summary of study features 43

$\begin{array}{ll}\text { Conclusion } & 45\end{array}$

$\begin{array}{lll}\text { Chapter Four: } & \text { Findings } & \text { The life long journey }\end{array}$

Introduction 46

My position in this chapter 46

$\begin{array}{ll}\text { My positioning of the Participants } & 48\end{array}$ 
The Participants $\quad 49$

$\begin{array}{ll}\text { Living with diabetes } & 51\end{array}$

The reality $\quad 54$

The struggles $\quad 56$

$\begin{array}{ll}\text { Family life } & 59\end{array}$

Making the transition to insulin therapy 62

The reality 65

$\begin{array}{ll}\text { The challenges } & 67\end{array}$

$\begin{array}{ll}\text { Turning points } & 69\end{array}$

$\begin{array}{ll}\text { Realisation } & 72\end{array}$

$\begin{array}{ll}\text { Seeing is believing } & 74\end{array}$

$\begin{array}{ll}\text { What insulin means for me } & 77\end{array}$

$\begin{array}{ll}\text { Conclusion } & 79\end{array}$

$\begin{array}{ll}\text { Chapter Five: } & \text { Discussions of the findings }\end{array}$

$\begin{array}{ll}\text { Introduction } & 80\end{array}$

Revisit Aims and Objectives of study $\quad 80$

Major knowledge contribution $\quad 82$

Description of the roof of the Samoan fale $\quad 82$

Symbolic meaning of the roof 83

The Grief Model $\quad 85$

$\begin{array}{ll}\text { Ia Malu Model (Description of) } & 87\end{array}$

Ia Malu Model (Illustration of) 95

Implications for Nurses and Health care professionals 96

$\begin{array}{ll}\text { Limitations } & 98\end{array}$

$\begin{array}{ll}\text { Future Research } & 98\end{array}$

$\begin{array}{ll}\text { Conclusion } & 99\end{array}$

$\begin{array}{ll}\text { Reference list } & 101\end{array}$ 
Appendices

Appendix 1

Central Ethics Approval

110

Appendix 2

Research Flyer

112

Appendix 3

Approval letter for study amendment

113

Appendix 4

Information Sheet (English version)

114

Appendix 5

Information Sheet (Samoan version)

115

Appendix 6

Consent Form (English version)

117

Appendix 7

Consent Form (Samoan version)

119

Appendix 8

Key Questions and Interview guide

121

Appendix 9

Transcriber's Statement

123 
List of Figures

$\begin{array}{lll}\text { Figure 1: } & \text { Fonofale Model } & 10\end{array}$

Figure 2: $\quad$ Ia Malu Model 96

\section{List of Tables}

$\begin{array}{lll}\text { Table 1: } & \text { Summary for search strategy } & 14\end{array}$

Table 2: $\quad$ Summary of study features 43 


\section{CHAPTER 1: Introduction to the Research}

Researchers have highlighted that Pacific people develop Type 2 diabetes much earlier in their lives (approximately 5 years earlier) when compared with New Zealand Europeans (Diabetes New Zealand, 2006). Indeed, Pacific people have poorer health status across a number of health and disability indicators, thus, exhibit a lower life expectancy compared with other New Zealanders (MoH, 2005; 2010). Type 2 diabetes is one of the major contributors to this health disparity (King, 2001), and as a consequence, Pacific people are at much higher risk of premature complications, including recurrent avoidable hospital admissions, poorer quality of life and untimely deaths (Foliaki \& Pearce, 2003; MoH, 2008).

The use of insulin to achieve acceptable glycaemic control in people with diabetes has been well established, for its clinical efficacy and in improved quality of life (United Kingdom Prospectus, Diabetes, Study (UKPDS), 1998). The unfortunate truth, as I have seen in my clinical practice, is that many people with Type 2 diabetes, who would benefit from using insulin, decline or refuse to use it. In addition, this group includes many Pacific people, who too choose not to use insulin. This has led to a rapid deterioration in their health. In this chapter, I will outline my research question, research aim and research objectives. I will also touch on my clinical role and introduce the Pacific health model that forms the theoretical framework for this study.

\section{Research Question, Aim and Objectives}

For the reasons mentioned above, Pacific people with Type 2 diabetes need to know that better management of their illness can be effectively achieved through the use of insulin. This notion provoked the need to search for solutions that may assist and guide my clinical work, in particular, with transitioning Pacific patients onto insulin therapy. There has been no documented work undertaken on how Pacific people, especially Samoans, come to terms with their transition to insulin therapy. This work is therefore critical in discovering this. Therefore, the research question then became: "How do Samoan people with Type 2 diabetes come to terms with their transition to insulin therapy?" 
The aim of the research is to explore and describe how Samoan people in New Zealand with Type 2 diabetes make their transition onto insulin therapy for the purpose of better glycaemic control.

The objectives of the study are:

1. To learn of Samoan people's experiences, beliefs and perceptions on what it means to be insulin users,

2. To learn about how Samoan people felt about commencing insulin and what they believe about the therapy,

3. To learn whether they have come to terms with being insulin users. If yes, how has this happened? If not, what has been happening?

4. To explore and uncover any personal struggles, hopes or desires that may have been experienced during the transition onto insulin therapy.

5. To discover any processes that may have helped or hindered them through these transitions.

An understanding of diabetes and its origin is critical due to the impact it has on people with the condition. The next section presents what diabetes is and its pathophysiological features, defining the differences between the known types of diabetes and its trajectory.

\section{What is diabetes?}

The correct medical term is diabetes mellitus (DM). Diabetes is derived from the Greek word which means "going through" and mellitus from the Latin word meaning "honey" (Porth \& Matfin, 2009). Throughout the text, DM and diabetes will be used interchangeably as both words are evenly used in the diabetes literature. The definition provided for DM by the World Health Organisation (2010) is that:

DM is a chronic disease caused by inherited and/or acquired deficiency in production of insulin by the pancreas, or by the ineffectiveness of the insulin produced. Such a deficiency results in increased concentration of glucose in the blood, which in turn damages many of the body's system, 
in particular the blood vessels and nerves (p. 1).

Although DM is a disorder involving insulin insufficiency, it is not a single disease. There are various forms of diabetes that have been arranged into categories, dependant on classifications with accord to stages of glucose intolerance. The two most commonly known forms of DM are Type 1 and Type 2. These were previously recognised as insulin dependent diabetes mellitus (IDDM) and non-insulin dependent diabetes mellitus (NIDDM). The changes in classifications are the responsibility of the Expert Committee on the Diagnosis and Classification of Diabetes Mellitus (Porth \& Matfin, 2009).

Effective diabetes management is indicated by an HbA1c of $7 \%$ or lower to minimise complication progression for the people with the disease (UKPDS, 1998). HbA1c is glycated haemoglobin that is used to describe haemoglobin into which glucose has been incorporated. Haemoglobin normally does not contain glucose when it is released from the bone marrow. During its life of 120 days in the red cell, haemoglobin normally becomes glycated to form a number of different haemoglobins, namely A1a, A1b and A1c. A1c

present in the blood provides an index of glucose levels over 6 to 12 weeks. In uncontrolled diabetes an increase in the level of A1c will be evident. An HbA1c of 7\% or lower is classified as normal glycaemic levels (Porth \& Matfin, 2009; UKPDS, 1998; Willams \& Pickup, 2004).

\section{Type 1 diabetes}

Type 1 diabetes is characterised by destruction of the pancreatic beta cells. It is thought to result from an interaction between genetic and environmental factors. This has led research into methods directed at prevention and early control of the disease; for instance, identification of genetically susceptible individuals and early intervention to control the disease. There is no trajectory of Type 1 diabetes because with the destruction of beta cells, people who develop it always require insulin therapy from the time of diagnosis to stabilise management. Type 1 diabetes is divided into two sub types: type $1 \mathrm{~A}$ immune mediated and type 1 B idiopathic (Porth \& Matfin, 2009; Williams \& Pickup, 2004). 
Type $1 \mathrm{~A}$ diabetes is commonly referred to as type 1 . It is characterised by immunemediated destruction of beta cells. It could have existed for years before the onset of hyperglycaemia. The slowly progressive form is known as latent autoimmune diabetes in adults (LADA). Type $1 \mathrm{~B}$ idiopathic is used to describe those "cases of beta cell destruction in which no evidence of autoimmunity is found. Only a small number of people with Type 1 diabetes fall in to this category" (Porth \& Matfin, 2009, p. 1056).

\section{Type 2 diabetes}

Type 2 diabetes is a heterogeneous condition. This describes the presence of high glucose levels in the association with relative insulin deficiency. Those who develop type 2 diabetes are often older and overweight. The metabolic abnormalities which lead to developing Type 2 diabetes include insulin resistance, deranged secretion of insulin by the pancreatic cells and increased glucose production by the liver (WHO, 2010; Williams \& Pickup, 2004). Type 2 DM is becoming more common among both obese adolescents and children (Porth \& Matfin, 2009), and it accounts for almost 90\% of diabetes cases (MoH, 2005).

\section{Trajectory of Type 2 diabetes}

Most patients with Type 2 diabetes eventually require insulin therapy because of the progressive nature of their disease and subsequent beta cell dysfunction (Porth \& Matfin, 2009; Williams \& Pickup, 2004). The traditional pathway for managing type 2 DM involves a stepwise approach that begins with lifestyle changes. Lifestyle modifications emphasises physical activity and dietary management. Failure to achieve acceptable management leads to the commencement of an oral hypoglycaemic agent (Eldor, Stern, Milicevic \& Raz, 2005). The United Kingdom Prospective Diabetes Study (UKPDS) (1998) showed that blood glucose control continues to deteriorate and subsequently in 2

years, a second, and eventual third agent are often needed. Only when all else fails is insulin added. By the time a person commences insulin, the average patient would have accumulated 5 years of HbA1c of $>8 \%$ (Eldor et al. 2005). The higher the HbA1c means the diabetes control is poorer. Therefore, the early use of insulin is essential to achieve improved acceptable control (UKPDS, 1998). 
A description of who I am as an individual and as a clinician follows. This is essential as it gives rise to the reasons for me to undertake such research.

\section{My clinical background}

I am a Pacific nurse of Samoan origin, who works in the specialised area of diabetes. My clinical workload is working primarily with Pacific people and with mothers with gestational diabetes in the region. I have been working in the field of diabetes for five years, which has contributed to my growing passion to continue working with Pacific people in addressing diabetes in the community. The area in which I work in is home to many Pacific families. Working together with the Pacific community is mostly important to help change the health status of our Pacific people. The majority of Pacific patients I have met in my work have Type 2 diabetes. Most of them already have secondary complications as a result of their illness. Working alongside these patients has opened my eyes to their daily struggles with diabetes and their strife for "normality". They have taught me meaningful insights into life, and how important it is to appreciate who we are, and what we are. I have found this role gratifying and rewarding.

Statistical information helps us understand the rise in numbers of Pacific people living in New Zealand. The next section focuses on profiling Pacific people.

\section{Profiling Pacific people}

People from the Pacific have been in New Zealand (NZ) for many years. The majority of them are here to stay (Tukuitonga \& Finau, 1997). Many moved here for greater opportunities and a better education for their children (Anae, 2009). Even as early as 1945, a small number of them already settled in New Zealand. At that time, they made up only $0.1 \%$ of the total New Zealand population. By 1991, they accounted for $5 \%$ of the total population (Statistics New Zealand, 1991). The trend in the number of Pacific people settling in New Zealand was clearly rising.

By the 2006 census, Statistics New Zealand (SNZ) reported that $8 \%$ of the overall national population identified as Pacific (SNZ, 2006); a total of 265,974 Pacific people living in 
NZ. Of that number, 131,100 were Samoans, who made up $49 \%$ of the total Pacific population (SNZ, 2006) and accounted for the majority of the Pacific population here in NZ. While Pacific people in New Zealand often share common migrant and assimilation history, many have retained their own unique languages, cultures and traditions (Tukuitonga \& Finau, 1997). Pacific people have made a massive contribution to New Zealand in the form of social, political, cultural qualities and sporting events (Wong, 2002). With these in mind, we also know that being here, impacts on the health system.

\section{Why this study is important}

Ten years ago, diabetes was listed as one of the 13 health priorities for NZ, because it has encroached on the lives of many New Zealanders, especially minority groups such as Pacific communities (King, 2000; 2001). Pacific people are dying younger and type 2 diabetes accounts for the majority of cases, resulting from diabetes secondary complications. These include cardiovascular diseases, end-stage renal failure and amputations ( $\mathrm{MoH} 2005 ; 2008)$. The $\mathrm{MoH}$ (2005) estimated an alarming 149\% increase of diabetes cases among Pacific people by year 2011 when compared with Maori and Europeans at $133 \%$ and $69 \%$ respectively. These socially and burdening complications can be delayed and improved through better management of diabetes (Nathan, 2007). Living with diabetes demands behaviour that motivates successful long-term adjustments to diabetes and its management (Dunn, Beeney, Hoskin \& Turtle, 1990). Included in this is the administration of insulin injections.

It is pivotal to remember that increasingly Pacific people are being diagnosed with Type 2 diabetes, which in turn is accounting for an accelerating rate of secondary complications and chronic co-morbidities. Achieving acceptable diabetes control is possible with intense insulin therapy. Therefore, increasing awareness and developing an understanding of the salient factors that impact on the decisions of the Samoan community towards insulin therapy, and how they come to terms with their therapy, is crucial in our work as diabetes nurses or health clinicians. The knowledge elicited from this study will not only help inform our practice, but will also generate valuable information that will prepare those that will follow us along the way within our discipline, to be better health promoters who in 
turn will advocate strongly for our communities at risk. This consciousness will help improve the delivery of care in the area of diabetes within the Samoan community - the population group who is at greater risk of diabetes and who in turn account for $49 \%$ of the Pacific population in New Zealand alone (Statistics New Zealand, 2006).

Commencing insulin is no easy task for the people needing it (Funnell, 2007). Often, the diverse beliefs and perceptions that patients have of insulin hinder successful transitions for better glycaemic control as proven by the Diabetes Attitudes, Wishes and Needs (DAWN) study (Peyrot, Rubin \& Lauritzen et. al, 2005). Insulin is the most effective treatment of diabetes (UKPDS, 1998); therefore, to remedy and reduce the complications imposed by type 2 diabetes on Pacific people, there is a need to introduce insulin as the treatment option early in their life-long journey with diabetes. Regrettably, in my clinical experience, many Pacific people who would benefit from insulin refuse to use it. Many of those whom I have seen are of Samoan descendent, possibly because the Samoan community in NZ is the largest Pacific group. Special attention to their beliefs and perceptions on insulin, should unpack factors that hinder their transition and use of it. This knowledge is exceptionally important in my clinical practice as it will make a difference to my practice, and the practice of other health care professionals (HCP). This knowledge will also form the foundation for future studies in Pacific health and chronic illness. There is no research to date on Samoan people's experiences of their transition to insulin therapy. This warrants such a study to learn more about these experiences.

The following section outlines the background to the research. It highlights why the research is important to finding out how people with diabetes transition onto insulin therapy for better glycaemic control.

\section{Background to this study}

Understanding the experiences, beliefs and perceptions of Samoan people with Type 2 diabetes in New Zealand of their transition and adjustment to insulin therapy, may enhance their uptake of insulin, resulting in improved future health outcomes and better quality of life. Pacific researchers understand the boundaries of their relationships to their own 
ethnicity and with other Pacific communities. It would be disrespectful to speak on behalf of another Pacific group (HRC, 2004); therefore, the recruitment of only Samoan participants for the current research project was a result of my cultural consciousness and sensitivity. It was more appropriate for me as a Samoan clinician and researcher, to engage with my people for this research (HRC, 2004).

As previously stated, insulin is the most effective treatment for diabetes in achieving better glycaemic control (UKPDS, 1998). These findings are encouraging for Pacific people and it is particularly important that good glycaemic control is gained and maintained to deter secondary complications and lead to improved quality of life. In my working experiences, I have witnessed many Pacific people who have declined or deferred commencement of insulin therapy, a treatment that would have given them better glycaemic control. The reasons that some would not use insulin were not always clear. This phenomenon got me enthused, and it is only by asking Pacific people with diabetes of their experiences that facilitates better understandings of their reasons. Psychological insulin resistance is a concept that covers factors like refusal of insulin (Bogatean \& Hâncu, 2004). There is a need to explore patients' beliefs of insulin to heighten knowledge and better approach the matter, particularly with minority groups and special populations such as Samoans.

The aim of the research is to explore and describe how Samoan people in New Zealand with Type 2 diabetes make their transition onto insulin therapy for the purpose of better glycaemic control. The only way we can ascertain this knowledge is by asking the Samoan people with type 2 diabetes of their experiences of their transition to insulin. Their individual stories themselves reveal factors that have future relevance for helping the Pacific communities, and other minority groups and patients with diabetes, in their transition to insulin therapy. This knowledge may lead to further research in the important areas of Pacific health and chronic illness.

The next section introduces a description and illustration of the Pacific model used to theoretically frame this research. The methodological approach used for the study follows later. 


\section{Theoretical Framework}

I have used a Pacific health model, the Fonofale model, created by Pulotu-Endermann (2001) for the purpose of my research. From the Fonofale model, I have chosen to focus and expand on the roof of the fale (house). By doing this, permits the weaving in of the framework to understand the experiences of the participants. As the roof shelters and protects the aiga (family), it too symbolises the cultural values and beliefs that shelter the family for life. The Fonofale Model developed by Pulotu-Endermann (2001) is a Pacific health model that embraces and incorporates the values and beliefs commonly shared by many Pacific people in New Zealand.

The illustration of a traditional Samoan fale (house) illustrates the make up of the Fonofale Model (see Figure 1), depicting the most important things that are commonly shared by the Pacific people. These values are family, culture and spirituality. I have specifically chosen this model because of these significant values and what they mean for Pacific people. I also chose the Fonofale because, the fale being a Samoan fale has sentimental value for me as a Samoan, a clinician and researcher in the area of diabetes and Pacific health. The use of this model forms a theoretical framing that values and respects the perspectives of Samoan people within this study on what being diabetic is like, and how they made their transition on to insulin therapy. 


\section{The Fonofale Model}

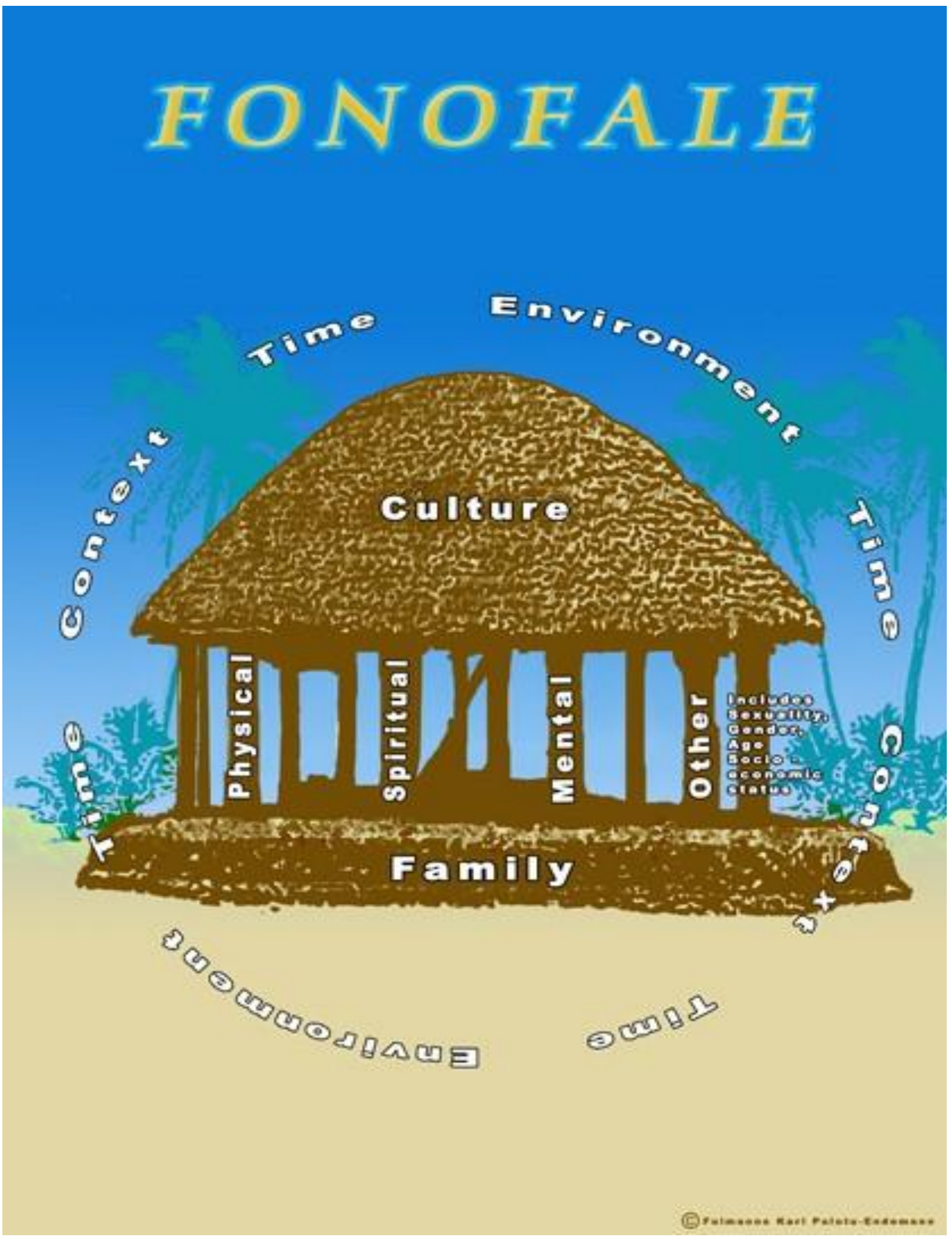

Figure 1: (Source: Reproduced with permission from Pulotu-Endermann, 2001.) 


\section{Introduction to the Methodology}

This research uses descriptive methodology with a qualitative approach and enables the researcher to focus attention to the experiences of Samoan people living with Type 2 diabetes through the use of in-depth interviewing. By using qualitative description this meant that the stories and experiences of the Samoan participants were then gathered to uncover their realities on being diabetic, as well as to how they made the transition to insulin therapy for better management. The participants' perspectives on the immeasurable challenges they encountered, provides us, as nurses and health care professionals, the best insights into their world with diabetes, as well as being insulin users.

There is no literature on how Samoan people with type 2 diabetes make the transition to insulin therapy. This highlights a gap in the literature that needs desperate attention. Descriptive methodology is the methodology of choice when the knowledge on a specific area is either limited or does not exist. Therefore, for the purpose of this research, and by employing qualitative descriptive methodology this will create knowledge and understanding essential to forming future research for Pacific health and chronic illness.

\section{Document Overview}

Chapter One introduces the research undertaken, outlining the research question, aim and objectives. A justification as to why this research is critical to the knowledge of nurses, but also for future health research on Pacific people and diabetes, is offered. I touch on the use of qualitative descriptive methodology as the methodology of choice for this research and introduce the Fonofale Model that formed the theoretical framework.

Chapter Two presents the review of literature, revealing practically no research on the subject. This chapter is arranged in three major sections that are critical to reviewing the subject understudied. There is a gap in the literature on how Samoan people make the transition to insulin therapy. A discussion on the two models is staged, arguing for the values they bring in forming the theoretical underpinning for the research. 
Chapter Three examines and discusses the methodological approach and study features of qualitative descriptive methodology, which is used for this research. This methodology is used when little or no knowledge exists on a topic. People stories are the prime sources of knowledge in qualitative research. The gathering of the participants' stories made us understand the experiences of the Samoan people of their transition to insulin. Face to face in-depth interviewing was used as the method for collecting data, and how the data was analysed is explained.

Chapter Four offers the analysis of the data findings. The participants are also introduced in this chapter and their narratives and personal quotes are used to augment the three major themes that emerged. Quotations from the interviews are presented in Samoan and English. The three major themes being: living with diabetes, making the transition to insulin and realisation, help us understand the experiences of Samoan people with type 2 diabetes of their transition to insulin therapy.

Chapter Five tables the discussions of the findings and conclusion to the research. The development of a model called the Ia Malu model helps us understand the experiences of the participants of their transition to insulin therapy. The weaving in of aspects of the Grief Model and the literature intensifies the meaning of the four phases that constitute the Ia Malu Model. Implications for HCP and limitations for the research are acknowledged in light of future studies directed by this piece of work.

\section{Summary}

This chapter introduces the research undertaken. Making the transition to insulin therapy is profoundly difficult among Pacific people with type 2 diabetes. Understanding the experiences of Samoan people of their transition will unpack factors that hinder the transition to insulin therapy. There is no research available on the subject being researched; therefore, the use of qualitative descriptive methodology was the most appropriate to generating knowledge and increasing awareness. This knowledge from the current study can form the basis for future research in the area of Pacific health and chronic illness. Chapter Two discusses the review of literature presented under four major sections. 


\section{CHAPTER 2: $\quad$ The Experience with Type 2 Diabetes}

\section{Introduction}

This chapter explores the areas that are pertinent to this research into Samoan's transition onto insulin therapy. They include diabetes and the implications it has on Pacific people's health. It evaluates existing knowledge on the transitions to insulin, and appraises concepts which embrace the values underpinning cultural differences and meaning. The chapter simultaneously weaves in the Fonofale Model (Pulotu-Endermann, 2001) to conceptualise the theoretical framework necessary for this study. This chapter is arranged in four major sections. These sections are:

1. The transitional journey to insulin therapy.

2. Implications of type 2 diabetes for Pacific communities.

3. Samoan people.

4. Cultural differences and meaning.

Before delving into the literature, I will present how I undertook the literature search. Table 1 summarises the search strategy. The table outlines the central topics, the search terms used and the databases searched for sources. It was critical to ensure all relevant literature was obtained to inform grounding for this study. Although the search retrieved useful literature on diabetes and treatments, I had to choose only the literature that was going to help inform the study. Having obtained all the relevant sources, they were categorised into research papers, commentaries and other. I had to keep connecting to my research question to ensure I did not exclude useful sources. 
Table 1: Table summarising the search strategy

\begin{tabular}{|l|l|l|}
\hline Central topics & Search terms & Databases searched \\
\hline Pacific Health & Diabetes mellitus, & OVID Medline \\
Health beliefs & Diabetes and (Samoa* or & CINAHL \\
Cultural & Cook Island* or Tonga* & PsychINFO \\
differences/diversity & or Tokelau* or Fiji* or & Sociological \\
Attitudes to health & Kiribati* or Hawaii* or & Abstracts \\
Transition to insulin & Niue) & Proquest \\
Knowledge transfer & & \\
Knowledge & & \\
synthesis & & \\
\hline
\end{tabular}

Having discussed the process carried out in retrieving my sources, staging the discussions related to Section 1 follows next. This section presents the transitional journey to insulin therapy.

\section{SECTION 1: $\quad$ The transitional journey onto insulin}

The review exposed virtually no evidence on how Pacific people make the transition on to insulin therapy when required for improved diabetes management. Insulin has been used since the 1920s for the management of diabetes mellitus and is a lifesaving therapy for patients with Type 1 (Williams \& Pickup, 2004). Insulin is also essential in attaining good diabetes control and reducing long term complications among many patients with Type 2 diabetes (Grunberger, 2009). Major diabetes studies, namely, the UKPDS (1998) and Diabetes Control and the Complications Trial (1993), quantified the relationship between glycaemic control and complications development especially microvascular complications. The evidence suggested that microvascular risks were reduced with tight glycaemic control of around $7.0 \%-8.0 \%$ and reducing HbA1c by $1 \%$ (Cartwright, Woodward \& Wallymahmed, 2006; New Zealand Guidelines Group, 2003). The efficacy of insulin therapy can assist anyone with diabetes to achieve tight glycaemic control.

Unfortunately, it is not always that easy for patients to agree when it comes to starting 
insulin, because it can be a relatively complex process in the people's minds. Nakar, Yitzhaki, Rosenberg and Vinker (2007) maintained that it is complex because the transition to insulin requires the knowledge and expertise of the physician, and the cooperation of the patient. Before the commencement of insulin, there is often psychological recoil from insulin, which leads to the patients refusing. However, only a few studies have reported results concerning psychological insulin resistance. In the UKPDS study (1998), 27\% of patients randomised to insulin therapy initially refused insulin compared to other groups. The Diabetes Attitudes Wishes Needs (DAWN) study also reported more than half of patients who had never used insulin experienced anxiety about commencing it (Peyrot et al., 2005). A study carried out by Khan, Lasker and Chowdhury (2008) with Bangladeshi patients for insulin refusal, is consistent with this notion. The participants expressed a reluctance to commence insulin. Bogatean and Hâncu (2004) referred to psychological insulin resistance as a concept that encompasses the different psychological factors a person faces when confronting chronic treatments like insulin.

Bogatean and Hâncu (2004) also identified, in their study of a group of 18 people with Type 2 diabetes in Romania, some of the most salient contributors to psychological insulin resistance. These include patients' personal view of reality of the reasons for starting insulin, perceived consequences of the insulin treatment and other factors such as family or others with diabetes, who contribute to the decision whether to refuse or accept insulin treatment. Identifying and addressing patients' beliefs about insulin just may address Type 2 diabetic patients' concerns about insulin. Addressing these concerns early in the treatment may help minimise, or inhibit psychological insulin resistance, and warrant additional study (Weinger \& Beverly, 2010).

Diabetes management demands a lifetime commitment to healthy behaviours that reduce complications. Effective communication between the patient and provider is of paramount importance, because of the power providers have over their patients (Lupton, 1995). Poor communication skills on the part of the patient or the provider can create barriers to adherence and treatment regimes. In a qualitative descriptive study undertaken by Mathews, Peden and Rowles (2009) the participants expressed that communication styles 
of the health care professionals influence the level of adherence to their regime. Communication could be another contributing element that hinders progress.

Psychological insulin resistance is an important factor worth investigating among people with diabetes who require insulin. It is a critical factor because of its bearings on the perceptions for the necessity to start insulin and consequences of the treatments (Bogatean \& Hâncu, 2004). I have found in my clinical experience as a diabetes educator that psychological insulin resistance does exist among patients. This makes this concept an important aspect in the care of, and management of diabetes among Samoan people. Therefore, this study is fundamental in exploring the experiences of Samoans when making the transition on to insulin therapy. Their stories may contain factors that impact on the acceptability of, and adjustment to, insulin therapy of Samoan people within New Zealand. Their personal journey and individual stories may contain within them factors that have future relevance for helping the Pacific communities, and other patients with diabetes, and facilitate further research in this important area of Pacific health and chronic illness.

Despite the gap in the literature concerning Pacific people and their transition to insulin therapy, there has been attention given to research related to lifestyle changes among Pacific people. Two New Zealand based research projects have involved testing a community based programme for diabetes risk reductions (Simmons, Voyle, Fou, Feo \& Leakehe, 2004; Swinburn, Amosa \& Bell, 1998). For example: The Ola Faautauta Project (Swinburn et al.,) and Tale of Two Churches (Simmons et al.,). The Ola Fa'autauta project was established as a community-based health promotion programme between three churches, to address the impact of nutrition, exercise and educational programme. The Tale of Two Churches compared the impact on weight and exercise of a diabetes risk reduction programme in two churches. Both studies concluded that such projects were invaluable because members of the intervention churches were ready to change. The next major section focuses on the health of Pacific people. 


\section{SECTION 2: $\quad$ Implications of Type 2 diabetes for Pacific people.}

\section{Health of Pacific people}

The health status of Pacific people is poorer across the spectrum when compared with the total NZ population. They include child and youth health, risk factors leading to poor health and long term conditions ( $\mathrm{MoH}, 2010)$. Pacific people also die young and have higher rates of long term conditions. Pacific people also develop Type 2 diabetes at a much younger average age than their European counterparts (MoH, 2005).

Diabetes is a lifelong progressive disease that is contributing to an exploding co-morbidity and death rates across the globe. It is the fourth or fifth leading cause of death in most developed countries, and Type 2 diabetes accounts for the majority of cases (Steyn, et al., 2003). Pacific people, like other indigenous and minority groups, experience a very high prevalence to type 2 diabetes that is thought to be driven by increasing obesity. Much is associated with changes in lifestyle, food intake and physical activity (Simmons, 1999). An article published in the NZ Herald (2007), featured headlines on Pacific obesity as being the worst in the world according to World Health Organisation (WHO). This article featured the top 10 ranks of the most overweight people in the world. Frighteningly, the top 8 out of the 10 fattest countries are in the Pacific, and Samoa was ranked $6^{\text {th }}$. Obesity has become a problem of poverty because people with little money have an easier time eating junk food and fill up on high caloric food (NZ Herald, 2007).

Like other parts of the world, the prevalence of Type 2 diabetes in New Zealand is increasing in alarming numbers. It is projected that the total number of cases between 1996-2011 will increase by 78\% (Robinson, et al., 2006). Hence, diabetes is a national concern and a health priority for the country. This trend has surely influenced enormous legislative efforts and attention within the government, to prevent and minimise cases in the most vulnerable population, such as Pacific people (MoH, 2008a).

Diabetes is very costly to a socialised health system, and there is already a rapid increase in this burden due to secondary complications such as premature renal disease and diabetic foot $(\mathrm{MoH}, 2005)$. This lead to the inclusion of diabetes as one of the 13 health objectives 
within the New Zealand Health Strategy (King, 2000) and priority in the Pacific Health Strategy (King, 2001). Health data for Pacific people has not showed much improvement over the last ten years. Pacific people still have higher risks of developing certain conditions and their health status remains poorer $(\mathrm{MoH}, 2010)$. Obesity and sedentary lifestyles are the contributing factors. To put it simply, their health statuses remain poorer and indeed, they are dying younger and have higher rates of long term conditions $(\mathrm{MoH}$, 2005). Samoan plays a significant role in the health of Pacific people within the New Zealand health system. Therefore, the following section describes who they are as a people and the fundamental aspects of the culture.

\section{SECTION 3: $\quad$ Samoan people}

\section{Samoan people in New Zealand}

Of all Pacific communities in NZ, Samoa makes up the majority. They made up $49 \%$ of NZ's Pacific population in the 2006 Census (Statistics New Zealand, 2006). Many Samoan people living in NZ migrated here in large numbers in the 1950s, and all for a number of good reasons. Many have settled in NZ in search of better education for their children and greater employment opportunities (Anae, 2009). Most of them arrived with no capital and were dependent on rental housing at that time. By 2001, two in three Samoans resided in the Auckland region; the next largest populations were in Wellington and Christchurch (Fairbairn-Dunlop \& Makisi, 2003).

Samoan churches also proliferated in NZ cities, which soon enough took on the role of villages. These church villages provided a platform for strong Samoan identity. Samoans are a deeply religious people and for many Samoans, Christianity and fa'asamoa (Samoan culture) are inextricably interwoven (Pitt \& Cluny, 1974). Christianity has become an important part of the culture, and the motto of Samoans is "Samoa is founded on God" (Meleisea, 1987, p. 70). 


\section{Samoan Culture}

The word fa'asamoa means culture in English. The concepts of the fa'asamoa are fundamental to the Samoan identity. These consist of a number of values and traditions. They are:

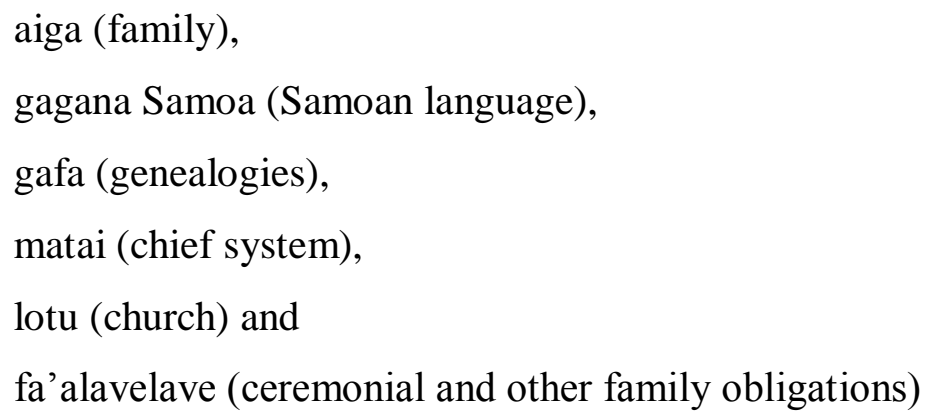

(Anae, 2009, p. 4).

Other important values that are also adhered to include: alofa (love), tautua (service), fa'aaloalo (respect), feagaiga (a covenant) and usita'i (discipline). The practices of fa'asamoa in Samoa may vary from that of New Zealand because of different understanding of the concepts. Despite living afar from families back in Samoa, close ties and links are constantly maintained (Fairbairn-Dunlop \& Makisi, 2003; Lay, 2000).

\footnotetext{
Aiga

Aiga means family. It is the primary unit of society in Samoa and the medium through which fa'asamoa operates. Aiga is the source of sustenance, certainty and comfort in times of social or economic troubles. Much inherent strength is present in extended family traditions (Lay, 2000). As the central element in Samoan culture, the aiga is the place where all other traditional values such as giving and receiving tautua (service), fa'aaloalo (respect) and alofa (love) are fostered, nurtured and practiced daily. Traditions are strongly maintained in Samoa and this is critical to ensure cultural continuity in Samoa's social relations. Money, prayers, support, food, material goods are also circulated within aiga (Lay, 2000), and as long as the aiga is functioning, "there is little need for childcare or home for the elderly" (Lay, 2000, p. 15). E. Tui Atua Tupua Tamasese (personal communication, November 3, 2009) spoke of the ideals of family within a Samoan context
} 
being shaped by respect for mental, physical, social and spiritual wellbeing for the individual; that being the responsibility of the aiga, ensuring each aiga member is happy.

The overview on Samoa and its culture is important in this context because of this research focus. Culture is not easily defined, because it is diverse and multifaceted. For Samoan people, the concept of culture only prospers when all elements mentioned in the above sections are adhered to, within respective aiga and social relations. Understanding the essential attributes of Samoan people may form awareness on some of the cultural meanings and differences it has as a minority group in NZ. In the next section an exploration related to cultural differences and meaning is offered.

\section{SECTION 4: The value of cultural differences and meaning for health}

Pacific people's health is shaped by cultural, social and economic factors (MoH, 2010). Many Pacific people have health beliefs, needs and realities that differ from other groups within NZ (MoH, 2008). Even if ethnic minority populations in our health community are small in numbers, it is critical to identify the causes that may affect the quality of their healthcare encounter. Some examples include linguistic needs and health beliefs (Davies, 2006). This section reviews the knowledge that underlines cultural differences as a disabling factor in health among minority groups and starts with a discussion of health beliefs.

\section{Health beliefs}

To successfully manage diabetes, an understanding of the beliefs, attitudes, family and social dynamics of the patient being treated is needed (Greenhalgh et. al, 1998). Health beliefs appear to have a major impact on people's adherence to treatment for diabetes per se, which in turn affect their health outcomes (Dunn, Beeney, Hoskins \& Turtle, 1990). In their study, Dunn et al, (1990) found that patients, who claimed diabetes to be more distressful and experienced difficulty coping with it after the diabetes education programme, had a $24 \%$ improvement in their HbA1c, compared to patients who reported a more positive attitude with only a $15 \%$ improvement. This indicates that improved diabetes control is often not without psychological cost. This result it would have seemed, was 
motivated by a change in behaviour towards better adherence to management and advice because there was evidence that suggested this. Thus, consistent with the assumptions of the Health Belief Model (HBM) that health related actions are taken when susceptibility and severity are perceived of illness (Glanz, Rimer, \& Lewis, 2002). The Health Belief Model is a psychological model that attempts to explain and predict health behaviours (Glanz, et. al, 2002).

Health beliefs are specific to individuals or groups. They can impact on diabetes control and predict health behaviours. The following subsection discusses the phenomenon of acculturation. The uncovering of knowledge correlated with cultural differences, exposed how acculturation could be responsible for some of the changes in health behaviour and morbidity, through culture change among minority groups.

\section{Acculturation}

In view of existing knowledge involving minority communities, the concept of acculturation, or assimilation, was also discussed in the literature (Corral \& Landrine, 2008). Acculturation is referred to as "the change in one culture in response to another" (Blakeley \& Dew, 2004, p. 1). Acculturation also refers "to leaving one's indigenous cultural context to spend increasing time in an alternative" (Corral \& Landrine, 2008, p. 737). For instance, Pacific people migrating to $\mathrm{NZ}$ become more prone to acculturation by losing traditional behaviours, while acquiring values of the dominant host culture (Corral \& Landrine, 2008). What effects it has on them we cannot know because of the limited body of available literature on this topic. What is known though, is the increase of acculturation on Pacific people (Blakeley \& Dew, 2004). Significant changes in health behaviour and morbidity are linked with increasing acculturation for all minority groups in White-dominant host cultures in countries such as the United States, Canada, Australia, New Zealand and Canada (Corral \& Landrine, 2008). Ethnic minorities who are among significant acculturation include Hispanic group, Asian minority, African Americans, American Indians (Corral \& Landrine, 2008). 
I would like to draw on what we do know about the impact of acculturation on minority groups. Take for instance the Hmong refugees in Minnesota, United States of America. These Hmong refugees came from Laos. They went through a difficult time to adjust to their new country, they did not know the language, they were not able to support themselves and bringing their children up in a country with different cultural values impinged on them and their health (Culhane-Pera, Her \& Her, 2007). In another example, the British Bangladeshi viewed exercise as potentially exacerbating illness and physical weakness; with many expressing "difficulty obtaining food that was both acceptable and palatable" (Greenhalgh et al., 1998, p. 980). In a study involving African-Caribbean people about their health beliefs, participants described migrating to the UK as stressful because of the process of change in culture, climate, poor housing and racial tension. Some participants felt that the stress, contributed to their onset of diabetes. Some believed that their predisposition to diabetes was exacerbated by having to adapt from Caribbean to British lifestyle (Brown, Avis \& Hubbard, 2007). These collective experiences are comparable to the Pacific studies. As a result, acculturation may be partially blamed for some of the changes in health behaviour and morbidity among minority groups (Davies, 2006).

For the purpose of this research, it is important to assess the literature on cultural differences because of bearing on health outcomes. The next subsection describes comparable studies that underline cultural differences.

\section{Appreciating cultural differences}

It is important to recognise and acknowledge that being different culturally itself (or cultural differences) do impact on health outcomes (Campos, 2007). It is also important to realise that cultural differences in health are due to more than just socioeconomic factors and explanations for these differences are many and multi-layered (Blakely \& Dew, 2004). In the literature, the term cultural differences is often affiliated with minority groups and related to cultural needs, being cultural sensitive and in some, culturally awareness (Davies, 2006; Greenhalgh, 1998). This notion is well noted in the field, but not for Pacific communities (Wang, Abbott, Goodbody, Wait-Ting \& Rausch, 1999). 
Pacific people have health beliefs that are different to those of other New Zealanders. These beliefs can influence health choices and behaviour ( $\mathrm{MoH}, 2008 \mathrm{a})$. Yet, there are limited studies that examine these health beliefs. The Pacific studies accessed, that are tabled in the next subsection, revealed findings congruent with what is reported in other studies concerning minority groups. These studies were conducted with minority groups like Hispanics (Walsh, Katz \& Sechrest, 2002), Hmong refugees in America (CulhanePera, Her \& Her, 2007), African-Caribbean in the United Kingdom (UK) (Brown et al. 2007), and British Bangladeshi (Greenhalgh et al., 1998). The overlapping commonality in all these minority studies and those reported in the Pacific focused studies, was the fundamental appreciation and recognition of the social and cultural differences of the minority communities. These research studies suggested that perhaps within these outcomes are crucial factors for implementation of culturally sensitive health services that best meet the needs of minority communities. Walsh et al., (2002) went further, and recommended that predictors of health serve merely as proxies to beliefs, values, resources and behaviour. They suggested that unpacking these proxies would result in more reliable interpretation and means of observing group differences otherwise.

Addressing the needs of any minority community requires an appreciation and recognition of cultural differences. It means providing culturally sensitive care appropriate to the needs of the respective communities. What is evident though is the implicit need to be providing care that embraces cultural differences. A discussion on the retrieved sources on Pacific people follows in the next subsection illustrating the cultural sensitivities of Pacific people.

\section{What are the Pacific people saying?}

Three qualitative research studies were accessed that described the need for culturally sensitive approaches when working with Pacific people. These studies were selected for their appropriateness and relevancy to Pacific people, and for this study.

One qualitative research study conducted by Moata'ane, Muimui-Heata and Guthrie (1996) investigated the understanding of 20 Tongan participants of diet and DM. Although the publication is almost 20 years old, the phenomenon studied remains relevant for Pacific 
communities today. The participants aged between 28 - 72 and had come from Tonga to live in NZ. The research revealed that Tongan's had traditional sets of beliefs that differ from those of western medical science. For example, they viewed food as the most visible expression of living and sharing, and to be told they had to restrict to a diabetic diet, was very difficult. The Tongan participants also perceived weight loss as a bad sign, because it was a "foreign" concept to them despite information given by the health care professionals.

Another similar study performed in NZ by Barnes, Moss-Morris and Kaufusi (2004), also focused on type 2 DM. Their aim was to determine whether there are cultural differences in the way in which Tongan and European people conceptualise their illness, treatment perceptions and adherence to self-care regimes. Their findings reported evidence suggesting that perceptions of diabetes differed between the ethnic groups. The elemental message emphasised here to health care professionals is to ask Pacific people of their beliefs about their illness, and not just questions of their knowledge and understanding. This study stressed the need to probe Pacific patients for their personal and cultural beliefs about their illness.

Overall, the two studies exposed the need to assess culturally sensitive factors in Pacific communities. The experiences of the Tongan people are representative of other Pacific groups because of their shared common Pacific heritage and cultural similarities. This opinion is further supported by Wang et al. (1999), who examined the perception of diabetes among a sample of Pacific people in Hawaii. Their findings reinforced that when researching Pacific people (including those living in Hawaii), it is essential to consider cultural differences in health promotion because of diverse cultural values and sensitivity embedded. The Pacific participants in their study revealed psychological factors such as anxiety, anger and having depressed mood as consequences of being diagnosed with diabetes.

On the whole, it is fundamental to solicit from Pacific people beliefs regarding their health and illness, rather than just obtaining their understanding and knowledge of it. What we know is that Pacific people's health beliefs are different to western beliefs. Therefore, 
culturally sensitive measures in diabetes healthcare will assist in achieving better health outcomes for Pacific people and other minority groups. The next subsection presents the Fonofale Model (Fuimaono, 2001), which provides the ideal theoretical framework for this study.

\section{The Fonofale Model}

I believe the Fonofale Model (Pulotu-Endermann, 2001) is the most culturally sensitive model for this study, because it characterises what it means to be Pacific. The Fonofale Model is a Pacific health model, developed in 1984 by Fuimaono Karl Pulotu-Endemann (see Figure 1). Its purpose was to capture what Pacific people viewed or believed to be the major determinants of their health. Consultations were carried out with the various Pacific communities to ensure all values and beliefs have been encapsulated in this one model. The values and beliefs central to Pacific people's health, and which are most important, are family, culture and spirituality. For that reason, the utilisation of the Fonofale Model (having incorporated all of the fundamental concepts meaningful to Pacific people), was the most logical, and most culturally sensitive. As highlighted in the literature, that to effectively address diabetes needs of minority groups, it is important to grasp a sense of cultural sensitivity to articulate differences.

The Fonofale Model encompasses the metaphor of a Samoan fale (Samoan house) to illustrate values and beliefs of the Pacific community. The fale consists of a foundation, roof and four posts. The Fonofale Model describes what each of these represent and what they mean for Pacific people in relation to health.

The foundation of the Fonofale Model represents the family, which is the foundation for all Pacific cultures. The family can be either a nuclear family, an extended family, or constituted family that are all connected by kinship, titles, marriage, partnership or covenance agreement. These aspects form the essential foundation of Pacific social organisations. Their history, genealogy or gafa, are within this foundation which enables links to titles, lands, islands, sea and to the Gods of the Pacific and other cultures. 
The roof of the fale symbolises cultural values and beliefs which shelter the family for life. Culture is a concept that is diverse, and thus persistently evolving and changing. In NZ, culture consists of NZ reared Pacific people, and those born and raised in the homeland. For some of these Pacific families, their culture may include practises that pertain to traditional Pacific norms, while for some, choose to live the Palagi (means European in Samoan) way and put in to practice Palagi values and beliefs. Others may live their lives right across the continuum between traditional Pacific cultural norms and Palagi cultural directions.

Holding the roof are the four posts, which stand securely in the foundation. These pou (posts) bind the culture and the family in a continuous and interactive manner. The four posts are:

Spiritual: - this post refers to the sense of wellbeing, which includes Christianity, or other traditional spiritual beliefs related to nature, spirit, language, ancestor and history, or a combination of both.

Physical: - this post relates to biological or physical wellbeing. It's the relationship of the body comprising anatomy and physiology, as well as the physical, organic or non-organic substances (i.e.) food, water, air and medications that can either impact positively or negatively.

Mental: - this post relates to the wellbeing or health of the mind involving thinking and emotions, with behaviours expressed.

Other: - this post relates to the many variables affecting health, whether directly or indirectly. These variables are but not limited to gender, sexuality, age, socioeconomic status.

The Fonofale Model is presented in a cocoon or circle containing these elements that may direct or indirectly impact one another. These are: 
Environment: - this dimension acknowledges the relationships and uniqueness of Pacific people to their physical surrounding. This could be rural or urban setting.

Time: - this dimension relates to the actual or specific time in history that impacts on Pacific people.

Context: - this dimension relates to the where/how/what and the meaning it holds for that particular person or people. Context could be in relation to Pacific people raised in the Pacific or in NZ. Other contexts include politics and socioeconomics. 
Figure 1:

The Fonofale Model

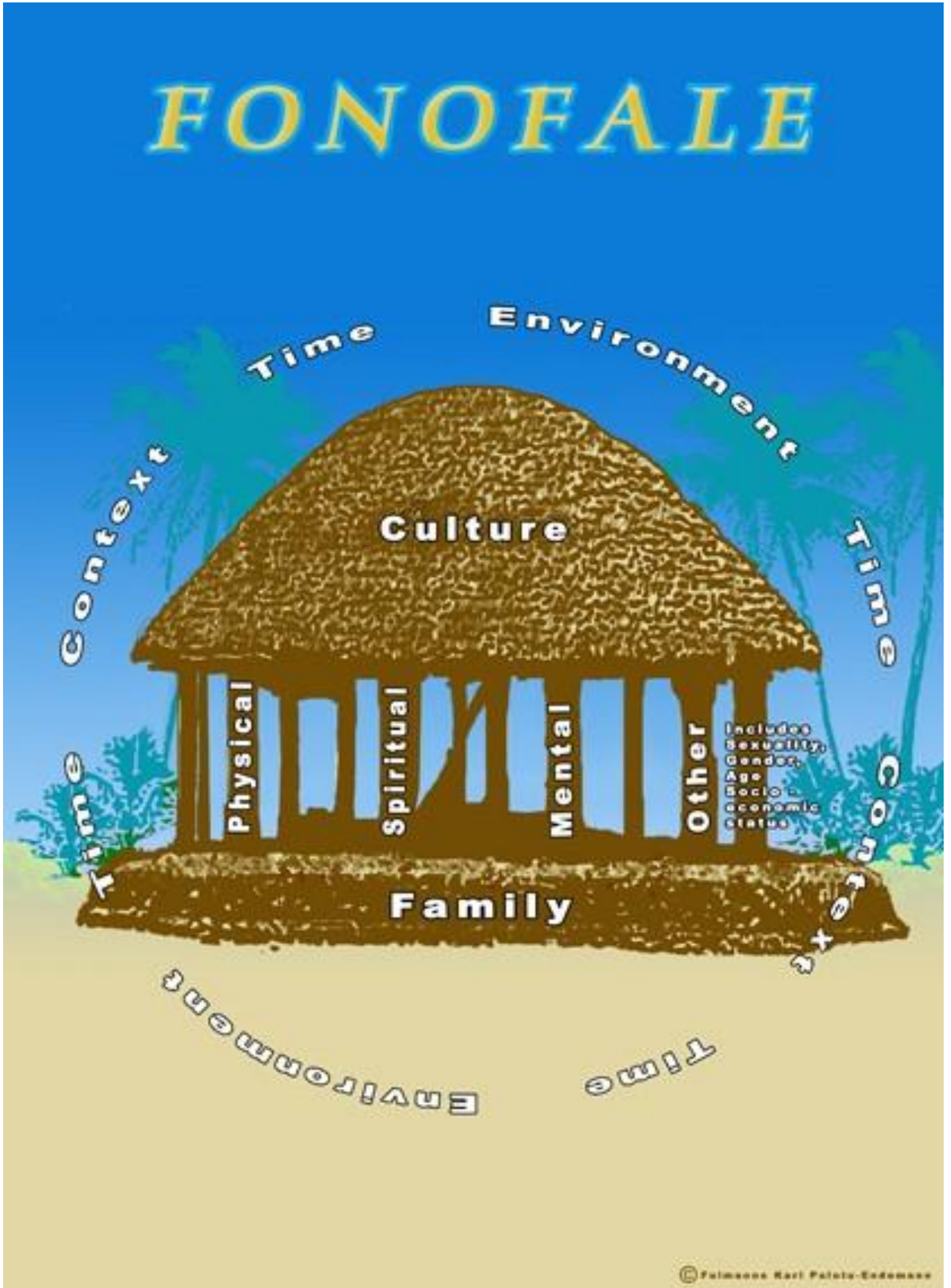

Figure 1: (Used with permission from F K. Pulotu-Endemann, 2001). 
The Fonofale Model best encapsulates the beliefs and values that are most important to Pacific people. The incorporation of the Samoan fale (house) is of significant value because it represents Pacific people. Therefore, this model was the most appropriate because of its culturally sensitivity and meaning for the Pacific community.

\section{Summary}

The review of literature exposed a gap in the field in terms of research concerning Samoan people with Type 2 diabetes and how they come to terms with their transition to insulin therapy. The consensus across other minority studies searching for similar answers demanded an appreciation of cultural differences and the need to provide culturally sensitive care. The utilisation of the Fonofale model to conceptualise the theoretical framework fundamental to this study was the most appropriate because of the way the model portrays Pacific values that are meaningful and significant to Pacific people. The Fonofale model illustrates the values and beliefs that are commonly shared by Pacific people, therefore, offering domains sensitive to them which include family, culture and spirituality.

This study employed descriptive methodology with a qualitative approach. The methodological approach and study design for this research are discussed in the next chapter. 


\section{CHAPTER 3: $\quad$ Research Methodology}

\section{Introduction}

It is essential to understand the experiences, beliefs and perceptions of Samoan people with Type 2 diabetes in New Zealand of their transition to insulin therapy. This understanding will provide diabetes educators and other health care professionals with a guide for assessing people's needs of similar populations. Gaining knowledge on the phenomenon of transitioning to insulin can be achieved by asking Samoan people with diabetes to speak of their experiences. People are a source of rich information because of their personal experiences and personal truths (Taylor, Kermode \& Roberts, 2006). Descriptive research is used when the knowledge on a specific area is either limited or does not exist (Sandelowski, 2000). This research uses the qualitative descriptive approach, because there is virtually no research done on the topic being researched. This chapter presents the key features of the research study design, ethical issues and methodological approach used.

\section{Aim and Objectives of the research}

The general purpose of this study was to generate knowledge and increase awareness on factors that relate to the acceptability and adjustment to insulin therapy of Samoan people with Type 2 diabetes residing in New Zealand. The aim of the research is to explore and describe how Samoan people in New Zealand with Type 2 diabetes made their transition onto insulin therapy for better glycaemic control.

The objectives of the study are:

- To learn of Samoan people's experiences, beliefs and perceptions on what it means to be insulin users,

- To learn about how Samoan people felt about commencing insulin and what they believe about the therapy,

- To learn whether they have come to terms with being insulin users. If yes, how has this happened? If not, what has been happening? To explore and uncover any personal struggles, hopes or desires that may have; during the transition onto insulin therapy, and 
- To discover any processes that may have helped or hindered them through these transitions.

\section{Research approach}

The review of literature presented in the previous chapter, reveals a dearth of information specific to Samoan people in New Zealand with Type 2 diabetes who require insulin therapy for better glycaemic control. Therefore, this research seeks to explore these experiences and perceptions regarding this transition process due to the limited specific knowledge. Qualitative descriptive research approach is the methodology of choice when little is known about an issue, or whereby a state of knowledge in an area is somewhat limited (Sandelowski, 2000; Trochim \& Donnelly, 2007).

The objective of qualitative descriptive research is to pay attention to conversations and describe the phenomenon with great richness. Researchers using qualitative descriptive not only stay close to the data but keep to the surface of words and events researched (Sandelowski, 2000). As a method, it characterises life as it is shared to amplify knowledge, and offer description of the patient's particular event that is being studied (Sandelowski, 2000). In addition, this form of research in nursing offers an opportunity to explore and examine patient's stories about health. The participants in the research shared their experiences about being diabetic and of their subsequent transition to insulin therapy; thereby, generating knowledge that may assist nurses and HCP in the way they work with Pacific people accessing health services. The generation of knowledge also supports policies, better targeting for resources utilisation and to change practice (Sandelowski, 2004). The generation of such qualitative description can deepen our social understanding of how things work, and the way people think and behave (Sandelowski, 2000; Trochim \& Donnelly, 2007). Qualitative research assumes that to understand human actions and behaviours, results in the need to understand the meaning and interpretations that people give to their own actions, to the actions of other and to situation and events (Hansen, 2006). 
By exploring the experiences of Samoan people with Type 2 diabetes in relation to their transition onto insulin therapy, rich data will emerge that may increase knowledge on the varied complexities experienced by these participants. Qualitative descriptive research is the research method of choice when straight forward description of the phenomenon is being desired and depending totally on the lived perceptions, sensitivities and inclinations of the describer. Sandelowski maintains that qualitative descriptive research demands the presentation of the facts of cases in everyday language. Qualitative descriptive research can offer researchers the flexibility to draw on other paradigms and philosophical positionings to capture as much data as possible (Sandelowski, 2000).

The uniqueness of qualitative descriptive research is specific to the kind of description it entails and the way it interprets the data. It offers a low-inference interpretation of the data generated when compared to other qualitative methodologies such as phenomenology and grounded theory. Qualitative research allows the researcher to remain close to the data, as well as keeping to the surface of the events being shared (Sandelowski, 2000; Trochim \& Donnelly, 2007). To support the achievement of validity, Sandelowski suggests that transcripts are returned to participants. She recommends that after a rich description of phenomena has been achieved that these are sent to the participants for general comments in relation to the accuracy of the events as they described them. Ideally, participants should be able to see aspects of their experiences in the description. It is fundamental for this study that the participants' stories were conveyed with meaning as they are told and experienced by the participants. Taylor et al. (2006) maintains that people are central to qualitative research because an understanding of their experiences can only be obtained through the expression of their life stories.

\section{Examples of qualitative approach in descriptive research}

Qualitative descriptive research has been described as the crudest form of inquiry, yet, the preferred methodology to build on nursing knowledge of events, where there is limited knowledge (Sandelowski, 2000). I would like to draw on two nursing studies that used qualitative descriptive methodology to generate knowledge that examine phenomena where there has been minimal previous knowledge. 
Dingley and Roux (2003) conducted a study on inner strength in older Hispanic women with chronic illnesses. They used a qualitative descriptive design and interviewed five women, whose experiences revealed the beginning basis of the structure, and process of inner strength in older Hispanic women living with chronic illnesses. Their study reported on inner strength in these women as a dynamic growth process extending throughout their lifetime, and a resource from which they draw on during illness and hardship. This study identified strengths that could contribute to theory development in advancing knowledge and intervention for guiding culturally sensitive practices and nurturing life of older Hispanic women with self management of chronic illness to incorporate into practice. Matthews, Peden and Rowles (2009) carried out a research study to describe the experiences, attitudes and beliefs of adult women who live with Type 2 diabetes. The number of participants recruited was five, and all were over the age of 50 years. The study identified three major barriers to the management of Type 2 diabetes: patient-provider communication, limited knowledge of diabetes and consequences of poor control of diabetes. The study highlighted the importance of patient-provider communication and attitudes in the management of diabetes. Some of the women in this study described office visits in which health care providers did not listen to their concerns and did not encourage questions. The women felt that such information is important in indicating that they felt satisfies with their treatment, empowered and prepared to actively take responsibility for disease management. The study concluded that the communication between the patient and provider cannot be stressed enough.

These nursing researchers have added knowledge to the existing body of literature on areas of health where limited prior research has been undertaken. Continuing nursing research in the areas of chronic illnesses and diabetes will continue to develop and refine models of care delivery to benefit and improve patient outcomes (Taylor et al., 2006). By using descriptive research, researchers have been able to create better understandings of the patient's realities. The next section presents the research design and description of the research features. 


\section{Research design}

The design of this research has been created from the key principles of qualitative descriptive methodology, and each feature will be discussed as follows. I have also used a table which follows later, to summarise each study feature and sum up the chapter.

\section{Overall feature of the study design}

Congruency in research is essential for assessing rigour and soundness, whether the method proposed is both appropriate and strong, relative to the issue being studied. It is essential to ensure congruency is accomplished between the foundational ideas and all the activity phases of the research, because it impacts on the quality and strength of the research. This means that the research question, aim of the study, method, data collection and analysis specific to this study are congruent. Rigour is referred to the strictness of judgement and conduct used to make certain the findings can be trusted. This means that the research is transparent and it "can be scrutinised by others for evidence of methodological accuracy and trustworthiness" (Taylor et al., 2006, p. 400). This simply means that the findings can be relied on, keeping in mind that it is less about getting at the truth but about meaningful conclusions, deeper understanding and useful results (Trochim \& Donnelly, 2007; Taylor et al., 2006). The categories for determining rigour are credibility, fittingness, auditability and confirmability (Taylor et al., 2006).

To assess for credibility, the participants and readers of the research are able to recognise the lived experiences described in the research as the same as their own. When considering fittingness, it means that the research findings need to fit into other contexts outside the study setting. Auditability is referred to the audit trail outlining decisions that are open for scrutiny of other researchers, determining whether consistency has been achieved in the research methods and processes. A research study has achieved confirmability when the first three categories can be demonstrated, having relied on the confirmation of participants, whose subjectivity is valued (Sandelowski, 2004; Taylor, 2006;).

This study achieved rigour by returning the transcripts to the participants for validation and verification of statements, and for them to be able to distinguish their stories within the 
findings. Keeping track of the decision making trail throughout the research was also fundamental so other health professionals could criticise it. Insights from the study specific to the participants' transitions to insulin therapy, are transferable to other contexts, and offer a platform for future research. The purpose for the research is to generate knowledge, and increase awareness on factors that relate to the acceptability and adjustment to insulin therapy of Samoan people with type 2 diabetes living in New Zealand. The study uses qualitative descriptive methodology due to the paucity of literature specific to the experiences of Pacific people in New Zealand, and how they make the transition onto insulin. The employment of this research approach is the most appropriate in order to discover the who, what and where of the events under study (Sandelowski, 2000).

\section{Why this research is important}

The motivation behind the research question was derived from the instances I have encountered in my professional experience as a diabetes nurse with Pacific people. I have seen in my practise that a number of Pacific people often decline to use insulin for better glycaemic control. Although I had some pre-determined ideas as to why Pacific people are declining insulin, developing a research question that asks this, will offer meaningful conclusions, deeper understanding and useful results for the generation of knowledge useful for the nurses/HCP (Trochim \& Donnelly, 2007; Taylor et al., 2006).

This study purpose is to generate knowledge and increase awareness of factors that relate to the acceptability and adjustment to insulin therapy of Samoan people with type 2 diabetes. Despite the importance of this issue, there is practically no research done on this research question, which highlights the real need to undertake this research now. The purposeful sampling is relative to this study, and as a result, this means that there can be no generalisation to other homogenous groups (Sandelowski, 1995; 2000). Additionally, the findings generated may inform and create platform for larger studies in this area of Pacific health and chronic illness, which have not yet been conducted. 


\section{Consultation}

Consultations for this study were fundamental in preparation for ethical approval (Appendix 1), and for the interviews with participants. I will discuss how this was undertaken for this study. Primary consultations took place with my supervisor through research supervision, to develop my research ideas, and establish succinct study objectives and aims. This process not only enabled me to progress and ground my research, but also supported and guided the research journey. Before the first interview took place, I was able to talk with my supervisor about my aide memoir during our practice run, which consisted of questions in the Samoan language that I translated for her. These practise sessions prepared me for my interviews, and reminded me of how important it was to keep a log book for reflections and impressions, after each interview took place.

Consultation with the Samoan community was another essential facet in the study. This study consulted the Samoan community through the Pacific Health Providers in the relevant $\mathrm{DHB}$, to enact respect, sensitivity and cultural safety for the wider community. The Pacific Health Services are seen and regarded as representing bodies and voices of the Pacific communities, and by consulting them I enhanced my safety as a researcher, but uphold cultural integrity for the community being researched. In addition, it was also crucial to obtain approval from the relevant DHB. This process is known as the locality assessment, which involved authorisation from the relevant $\mathrm{DHB}$, prior to recruitment of participants from within the relevant region (HRC, 2004).

\section{Treaty of Waitangi}

This study respects and acknowledges the existence of the Treaty of Waitangi in Aotearoa, New Zealand. It is the responsibility of the researcher to ensure the research undertaken, values the three key principles of the treaty being partnership, protection and participation (Durie, 1998). For all research carried out in New Zealand, researchers need to highlight in their research proposal any implications of their research for tangata whenua (Maori people) (HRC, 2004). 
Although this research focused on the health of Samoan people in NZ with Type 2 diabetes, I had to make sure that I uphold my responsibility as a researcher to demonstrate and show respect for the tangata whenua - the indigenous people of NZ. To address this issue, consultation was carried out with the Research Advisory Group - Maori (RAG-M) in the region, to reassure that the safety of Maori people is maintained. During consultation, I was asked what I would do if one of the participants was Maori, I highlighted, that to be recruited for this research, the person must identify as Samoan otherwise, they would not fit the research inclusion criteria (Appendix 2).

\section{Inclusion criteria for recruitment}

The research participants needed to identify as Samoans, aged 18 years and over, have Type 2 diabetes, have commenced insulin in the last twelve months and reside at the relative DHB (Appendix 2). I purposely did not recruit from the DHB I work for, because of any biased opinion or conflict of interest (outside the DHB I work in). With this recruitment guideline, I only managed to recruit two participants. There were two other people interested in the research, but they did not fit the inclusion criteria because they both had been using insulin for over four years. I struggled to recruit under the original guideline, and I corresponded with the Ethics Committee about changing the inclusion criteria, in which ethical clearance was given (Appendix 1) and it therefore, changed from 12 months to 36 months since using insulin (Appendix 3). I later recruited two more participants who fitted the criteria, and who were keen to share their stories. The research included a total of four participants. Details on the recruitment process follow next, describing how the participants were recruited for the study.

\section{Recruitment process}

The participants for the study were invited to freely choose to participate through research flyers (Appendix 2) that were distributed to the medical centres and hospital, and with the help of nurses/HCP which included information on how to contact the researcher (Appendix 4). The information sheets were made available in both the Samoan and English languages (Appendix $4 \& 5$ ) to ensure that the potential participants fully understood the study objectives and what it required of them. Direct contact was made with interested 
participants on the phone, to discuss a suitable time to talk about the study aim and objectives. These discussions were held at the homes of the potential participant's to maintain respect and cultural values (HRC, 2004).

The recruitment process was gradual. My first interview was completed almost two months before the second one. I then went on the Samoan radio to further talk about the research, in the hopes that interested participants would contact me. Only one interested participant came forth after the radio talkback, and we followed the process as planned in the two scheduled meetings. The initial one was to explain the study and go through the information sheet. The keen participants was given time (at least a week) to consider taking part. I then contacted the potential participant later on the phone to follow up consent and to arrange and set appointments for the interviews at their homes. Although I had consulted the Pacific providers in the area beforehand, I also needed to make sure that each participant was properly informed to consent (Appendix 6 \& 7) (HRC, 2004) to ensure their safety. These consent forms were made available in the Samoan and English languages for the participant's safety (Appendix $6 \& 7$ ). The fourth participant was recruited via a hospital clinic.

\section{Data collection, sources and methods}

In-depth interviewing with open-ended questions was the means for data collection for this research. These were done face-to-face with the participants, with each interview lasting between 60 to 90 minutes. These took place at the participants' homes at the times they agreed on. Minichiello, Aroni and Hays (2008) pointed out that when we are engaged in indepth interviewing, it is the people's experiences that we are actually interested in, of their social reality through their own interpretation of it. In-depth interviews are like conversations where participants express themselves in a "flow of consciousness" and in fitting with epistemological assumptions that truth are relative and context dependent (Taylor et al., 2006). The purpose of the interview is to probe the ideas of the interviewee(s) about the phenomenon of interest. The use of in-depth interviewing is based on acknowledging that: 
The world of nature as explored by the natural scientist does not mean anything to molecules, atoms and electrons. But the observational field of the social scientist - social reality - has a specific meaning and relevance structure for the beings living, acting and thinking within it. (Schütz, 1962, p.49)

In-depth interviews were carried out for the four Samoan participants at their homes, which were recorded and later transcribed. As Sandelowski (1995) claimed, qualitative approaches typically involve purposeful sampling that is case-oriented, rather than variable-oriented. Like my research, although the sample size was small, it still provided sufficient data key to identifying the core themes (Sandelowski, 2000).

Given that this research involves researching Samoan people whose first language is Samoan, three of my four interviews were conducted in Samoan as this was the preferred language for the participants - only one interview was carried out in English. This gave direction to this research and the Samoan language narratives are valued in the findings chapter that follows later. The feature of using Samoan narratives in the findings chapter embodies not only the cultural appropriateness of the Samoan participants but express cultural sensitivity unique to each life story of the participants as they each share their experiences of being diabetic and how they transitioned on to insulin therapy. My rationale for deciding to bring in Samoan texts from the interviews is primarily to provide us with lens and light into the participants' world. This allows us to holistically see what those experiences and the realities of living with diabetes for the rest of their lives in relation to the experience of transition on to insulin therapy. Sandelowski (1994) emphasised the growing recognition in narratives as "a means to achieve what is unique to nursing (p. 24) and to discover and to uncover knowledge, as well as recovering the art of nursing.

On the day, before the interviewing began, once informed consent (Appendix $6 \& 7$ ) has been obtained, the demographic data was then collected. This process allowed us to develop an informal rapport, which then progressed smoothly to the interview. The individual interview started by asking the participant to: "Tell me what it's been like for 
you since you were diagnosed with diabetes?" The interview concluded by asking each participant: "Would you like to add anything else; what advise would you give to the Samoan community out there?" I have included key questions used at the interview (Appendix 8). These interviews were recorded and transcribed at the end. Three of the transcripts were in Samoan which I transcribed myself, while one in English was transcribed by a transcriber who signed a confidentiality form prior transcribing (Appendix 9). Recording each interview helped ensure all the data described were collected, but it also provided an instrument to capture the participant's words to be used in the analysis (Taylor et al., 2006). The next section describes the study ethics and its considerations.

\section{Ethics}

Researchers working with human sources must remember that participants are real, with own realities, experiences and uniqueness (HRC, 2004). In addition, respect for humanity and participant safety must be maintained at all times. This means full disclosure of research processes and study purpose are given to the participants. This study could not progress with participant recruitment until it received full ethical clearance and approval from the Ethics Committee. To keep within the ethical guidelines approval was sought and obtained from the Central Regional Ethics Committee (Appendix 1).

Since this study recruited Samoan adults whose first language is Samoan, it was essential to ensure that all the research materials such as the consent forms and information sheet were also made available in the Samoan language (Appendix 5 \& 7). The Ethics Committee ensured they had seen these beforehand at the time of ethical resubmission, to guarantee the safety of the community members involved in the research. The participants' confidentiality was maintained at all times throughout the research, and the information used was no way identifiable for any of them. They were well informed of their rights to withdraw from the study at any given time (see Appendix 4, 5, $6 \&$ 7). The HRC (2004) emphasised that "researchers must uphold the right of the participants to withdraw" safely and with dignity (p. 34). It is important to ensure that the principles of beneficence and non-maleficence, which underpin research standards, were adhered to, to keep the participants safe (Taylor et al., 2006). Pseudonyms are used to preserve the participants' 
identities. These pseudonyms are Sina, Sione, Emma and Pua. Their interview transcripts and copies of the raw data are being kept in a locked cupboard for the mandatory period of ten years, and only to be accessed by the principal researcher and supervisor. Consent was also sought from the participants for this component in case of future studies to be conducted within a bigger research sample (Appendix $6 \& 7$ ).

I had a cultural mentor who provided me with cultural support and guidance, and also rechecked the translations of the narratives I used in the analysis to ensure the meanings as intended by the participants are maintained. When the transcripts became available, I went through them again with each participant face-to-face, to confirm their statements and check for accuracy. Sandelowski (2000) asserted that researchers conducting qualitative descriptive studies must seek descriptive validity to check for accuracy of accounts.

\section{Data analysis}

During the analysis, I worked from the three Samoan transcripts and one English one. Qualitative thematic analysis was used to systematically analyse and code the data that was gathered. Careful coding of the data was applied to refine core themes across the four transcripts that fit best in relation to the research aims and objectives. Sandelowski (1995) insisted that "qualitative analysis is a means to knowledge edge production that involved the separation of elements of data according to some priori or data-derived system" ( $p$. 372). I began with the re-reading, re-looking and re-listening to each transcript, to familiarise with the nuances within them. I immersed myself within the texts with alertness, in the search for the theme to isolate key themes. I did this by noting the themes as they occurred for each transcript. These were then organised to key themes as they recurred for all the four participants. What was obvious and beginning to form was a picture of what living with Type 2 diabetes is like for the participants, and of their moving experiences when they transitioned to insulin therapy.

To make sure I was keeping within the essence of this research, I had to continuously check and recheck my research proposal for the research aims and objectives. The expected result of qualitative descriptive research is a straight forward description of the 
data contents (Sandelowski, 2000). The major themes generated unpacked and uncovered the experiences of Samoan people with Type 2 diabetes of their transition to insulin therapy. These will be discussed in the next chapter.

\section{Consideration of rigour}

There are a number of ways I have drawn on to ensure rigour and validity for the research. People are the prime sources of knowledge in qualitative research because of their life stories and subjectivity. This sort of knowledge cannot be generalised because it is not true for everyone and it is context dependent (Taylor et al., 2006). Credibility was achieved by returning the transcripts and going through them with the participants to verify statement contents and to check for accuracy. The participants were able to distinguish their stories in the findings. The research also has the ability to be transferred to other meaningful contexts outside this study setting, forming a platform for future studies in the issue understudied for Pacific health and chronic illness. The careful and systematic coding of the transcripts during data analysis refined and isolated the major themes that reflected the experiences of the participants. The narratives of the participants used in the analysis have been clearly labelled to easily identify the excerpts. This provides an audit trail.

The use of a cultural mentor ensured that the English translations were done in a manner that it did not alter the meaning as intended by the participants. This was critical to assure that there was no data distortion. I have described the decisions I undertook through the research to ensure the methods remained thorough during data collection. For example, the change that took place for the study inclusion criteria. This allows the study trail to be scrutinised by other researchers in determining whether consistency has been achieved throughout.

In this next section, I have included a table (Table 2) to summarise the overall study design and key features. I have done this because I wanted to offer a brief overview and outline of the study design and key features to round up the chapter. The key headings used in the table are: Research feature, methodological strategy, study design, theoretical rationale and congruence as described by Sandelowski (2000), to rationalise and demonstrate 
congruence between the study and methodological approach used by ensuring the research has achieved credibility, fittingness, auditability and confirmability (Taylor et al., 2006).

Table 2: $\quad$ Overall summary of study features.

\begin{tabular}{|c|c|c|}
\hline $\begin{array}{l}\text { Research } \\
\text { features }\end{array}$ & $\begin{array}{l}\text { Methodological strategy and } \\
\text { study design }\end{array}$ & $\begin{array}{l}\text { Theoretical rationale and } \\
\text { Congruence }\end{array}$ \\
\hline $\begin{array}{l}\text { Research Aim } \\
\text { \& Objectives }\end{array}$ & $\begin{array}{l}\text { To generate knowledge and } \\
\text { increase awareness on factors that } \\
\text { relate to the acceptability and } \\
\text { adjustment to insulin therapy of } \\
\text { Samoan people living in New } \\
\text { Zealand (NZ) with Type } 2 \text { diabetes } \\
\text { (T2D). }\end{array}$ & $\begin{array}{l}\text { To listen and learn from the } \\
\text { participants of their experiences } \\
\text { beliefs and perceptions of this } \\
\text { process. }\end{array}$ \\
\hline $\begin{array}{l}\text { Research } \\
\text { approach }\end{array}$ & $\begin{array}{l}\text { The study uses qualitative } \\
\text { descriptive highly influenced by } \\
\text { the work of Sandelowski (2000). }\end{array}$ & $\begin{array}{l}\text { Method of choice when straight } \\
\text { descriptions of the participants' } \\
\text { experiences, beliefs and } \\
\text { perceptions of their transition } \\
\text { onto insulin therapy. } \\
\text { The approach is more appropriate } \\
\text { for generating answers important } \\
\text { for health decisions. } \\
\text { Generation of valuable primary } \\
\text { end products as well as secondary } \\
\text { entries for future research. }\end{array}$ \\
\hline $\begin{array}{l}\text { Why this } \\
\text { research } \\
\text { question? }\end{array}$ & $\begin{array}{l}\text { Virtually nothing is available in } \\
\text { NZ on people with Type } 2 \\
\text { diabetes of their transition onto } \\
\text { insulin therapy let alone for Pacific } \\
\text { people. }\end{array}$ & $\begin{array}{l}\text { Findings essential to uncover and } \\
\text { understand how Samoan people } \\
\text { with Type } 2 \text { diabetes in NZ make } \\
\text { their transition onto insulin } \\
\text { therapy, and also important for } \\
\text { me as a Pacific nurse working in } \\
\text { the area of diabetes. }\end{array}$ \\
\hline
\end{tabular}




\begin{tabular}{|c|c|c|}
\hline Consultations & $\begin{array}{l}\text { Primary consultation through } \\
\text { Research Supervision. } \\
\text { Consultation with Samoan } \\
\text { community through Pacific Health } \\
\text { Services (PHS). } \\
\text { Consultation with Maori } \\
\text { representatives. } \\
\text { Locality Assessment with relevant } \\
\text { DHB. }\end{array}$ & $\begin{array}{l}\text { To enhance safety and integrity of } \\
\text { the researched community and its } \\
\text { process. } \\
\text { Enact respect, cultural sensitivity } \\
\text { and cultural safety. } \\
\text { To safeguard participants as well } \\
\text { as researcher. }\end{array}$ \\
\hline $\begin{array}{l}\text { Treaty of } \\
\text { Waitangi } \\
\text { implications }\end{array}$ & $\begin{array}{l}\text { Valuing the three principles of the } \\
\text { Treaty: Protection, Participation } \\
\text { and Partnership. } \\
\text { Consultation with Research } \\
\text { Advisory Group - Maori (RAG- } \\
\text { M). }\end{array}$ & $\begin{array}{l}\text { Valuing and respecting the } \\
\text { Treaty of Waitangi. } \\
\text { Adhere to culture safety } \\
\text { principles which are Protection, } \\
\text { Participation and Partnership. } \\
\text { Ensuring safety of researcher. }\end{array}$ \\
\hline $\begin{array}{l}\text { Inclusion } \\
\text { criteria for } \\
\text { recruitment }\end{array}$ & $\begin{array}{l}\text { Samoan adults > age of 18years. } \\
\text { Has Type } 2 \text { Diabetes. } \\
\text { Commenced insulin within last } 12 \\
\text { months (Appendix 2); later } \\
\text { changed to } 36 \text { months with ethical } \\
\text { committee approval (Appendix } 3 \text { ). } \\
\text { Resides in the area of the relevant } \\
\text { DHB. }\end{array}$ & $\begin{array}{l}\text { Manageable small sample scale to } \\
\text { maintain study time frame; } \\
\text { Recruitment with the relevant } \\
\text { DHB at both Primary and } \\
\text { Secondary care. }\end{array}$ \\
\hline $\begin{array}{l}\text { Recruitment } \\
\text { Process }\end{array}$ & $\begin{array}{l}\text { Recruiting using research notices } \\
\text { and advertisements with the help } \\
\text { of Health professionals (Appendix } \\
\text { 2). } \\
\text { Direct contacts with interested } \\
\text { participants. } \\
\text { Initial meeting to explain study. } \\
\text { Once consent (Appendix } 6 \& 7 \text { ), }\end{array}$ & $\begin{array}{l}\text { Participants freely choosing to } \\
\text { participate. } \\
\text { To share experiences and beliefs. } \\
\text { Maintain cultural safety and } \\
\text { sensitivity. }\end{array}$ \\
\hline
\end{tabular}




\begin{tabular}{|c|c|c|}
\hline & second meeting for interview. & \\
\hline $\begin{array}{l}\text { Data } \\
\text { collection, } \\
\text { sources and } \\
\text { methods }\end{array}$ & $\begin{array}{l}4 \text { Samoan adults. } \\
\text { Individual interviews using in- } \\
\text { depth interviews. } \\
\text { Interviews recorded and } \\
\text { transcribed. } \\
\text { Pre-designed questions (for key } \\
\text { questions please see Appendix 8). }\end{array}$ & $\begin{array}{l}\text { Purposeful sampling due to small } \\
\text { scale study. } \\
\text { Manageable size that should } \\
\text { support sufficient data and } \\
\text { identifications of the main core } \\
\text { themes. } \\
\text { Data to ascertain the what and } \\
\text { why specific to transitioning onto } \\
\text { insulin. }\end{array}$ \\
\hline Ethics & $\begin{array}{l}\text { Ethical considerations and ethics } \\
\text { Approval (Appendix 1). } \\
\text { Maintain participant's safety and } \\
\text { confidentiality. } \\
\text { Participation is totally voluntary. } \\
\text { Informed consent obtained } \\
\text { (Appendix } 6 \& 7 \text { ). } \\
\text { Information sheet provided } \\
\text { (Appendix } 4 \& 5 \text { ). } \\
\text { Transcripts and study results } \\
\text { available for the participants. } \\
\text { These were returned to } \\
\text { participants for rechecking and } \\
\text { validating. }\end{array}$ & $\begin{array}{l}\text { Ethical clearance for study } \\
\text { attained from Regional Ethics } \\
\text { Committee (Appendix 1). } \\
\text { Adequate information (Appendix } \\
4 \text { \& 5) given for participant's } \\
\text { prior consenting to participate } \\
\text { (Appendix } 6 \text { \& 7). } \\
\text { Explain risks and benefits of the } \\
\text { study. } \\
\text { Maintain participant's safety. } \\
\text { Keep sensitive data safe. }\end{array}$ \\
\hline Data analysis & $\begin{array}{l}\text { Qualitative thematic analysis. } \\
\text { Systematic analysis and coding of } \\
\text { each transcript to identify common } \\
\text { themes. }\end{array}$ & $\begin{array}{l}\text { To present data in everyday } \\
\text { language. } \\
\text { Careful and systematic coding of } \\
\text { the transcripts (i.e.) common } \\
\text { themes between all participants } \\
\text { (e.g.) on living with diabetes, } \\
\text { experiences with using insulin, }\end{array}$ \\
\hline
\end{tabular}




\begin{tabular}{|c|c|c|}
\hline & & what insulin means to them. \\
\hline $\begin{array}{l}\text { Consideration } \\
\text { of rigour and } \\
\text { trustworthiness }\end{array}$ & $\begin{array}{l}\text { Systematic strategy to assess for } \\
\text { validity and reliability of research. } \\
\text { Credibility assessment - } \\
\text { Participants to validate transcripts. } \\
\text { Participants to verify findings. } \\
\text { Peer review of findings. } \\
\text { Fittingness/Transferability - } \\
\text { Describing context central to } \\
\text { research and as told by } \\
\text { participants. } \\
\text { Auditability and Confirmability - } \\
\text { providing decision trail. }\end{array}$ & $\begin{array}{l}\text { Ability to make sense and keep } \\
\text { track of the decision making trail. } \\
\text { A log book was kept for } \\
\text { reflection and for writing in } \\
\text { impressions after every interview. } \\
\text { Participants distinguishing their } \\
\text { stories in the findings. } \\
\text { Ability to transfer findings into } \\
\text { other meaningful contexts outside } \\
\text { study setting. } \\
\text { Findings that provide insights } \\
\text { specific to transition to insulin, } \\
\text { and offer a platform for future } \\
\text { reinvestigations. } \\
\text { Careful and systematic coding of } \\
\text { the transcripts. } \\
\text { Qualitative strategies that } \\
\text { support trustworthiness and } \\
\text { credibility of the results such as } \\
\text { going through transcripts with } \\
\text { each participant for validation. }\end{array}$ \\
\hline
\end{tabular}

\section{Conclusion}

This research study used qualitative descriptive methodology. The use of in-depth interviews to collect the sensitive data from the Samoan participants was the most appropriate to use because it probed the phenomenon under study. The careful examination of the data using thematic analysis was used to extract themes. I am going to explore these themes in the next chapter; these major themes help us understand the experiences of Samoan people with Type 2 diabetes on how they made the transition to insulin therapy. 


\section{CHAPTER 4: Findings: The life-long journey}

\section{Introduction:}

This chapter reports on the findings that have emerged from this research. To understand the participant's journey with Type 2 diabetes, and their coming to terms with insulin therapy, three major themes and relevant subthemes were extracted from the transcripts. These themes recurred throughout the interviews, intensifying connections with the objectives of this research. These major themes were revealed:

(1) Living with diabetes

Reality

The struggles

Family life

(2) Making the transition onto insulin therapy

The reality

The challenges

The turning points

(3) Realisation

Seeing is believing

What insulin means

This chapter presents the themes in the order outlined above. The participant's narratives are used to exemplify each experience and story to keep within the qualitative descriptive research method. The Samoan narratives and personal quotes used enhance the participants' interviews, and individualise and capture the specialness of each story told. The decision to present quotations initially in Samoan is to add richness, as the essence of what is meant can be lost in translation. For each Samoan narrative, an English translation is provided. The translation was done for the purpose of the research results.

\section{My position in this chapter:}

I experienced some cultural tension in writing this chapter. Culturally, it would have been more appropriate to present each participant and their stories independently, especially when attention is given to the personal encounters, stories and realities of Samoan people. 
In doing so, would not only enrich uniqueness for each participant, but would also magnify the cultural specialness pertaining to each one. Unfortunately, the style of academic writing, and my methodology, require that I present them in a more empirical way. Vaioleti (2006) discussed the strictness of traditional research methods that Pacific researchers must adhere to generate knowledge. The impact of this on Pacific researchers means sanitising of cultural and spiritual values, and deep cultural concepts, that perpetuate Pacific peoples' realities in Aotearoa. So as a result of this tension, each participant is introduced on their own, but their narratives are later aligned together, to conform with western academic conventions.

Participants are considered to be unique individuals who are involved in research. Their stories and experiences provide us with a lens to see into their world and reality (Sandelowski, 1994). The participants in this research relaid their individual realities. Their accounts conveyed interesting events in their journey with diabetes, as well as their subsequent transition on to insulin therapy. Sandelowski (1994) maintains that the patient's "stories provide a sense of connection to other people.... and between life events" (p. 26) as they retell and recapture their struggles with disruptions of illness or transition. For that reason, I need you to know that I respect and value each individual encounter. I was priviledged to have each story gifted to me as a researcher. We are therefore, also honoured to be the listeners and receivers to these incredible stories.

To ensure that I remained culturally and academically safe, I decided to keep within the qualitative description presentation of my findings as expected within the academic realm for research. I eventually came to the realisation that despite my uncomfortable tension, a much stronger purpose in reporting of our uniqueness and specialness together was by far the most critical. This is because when the participants stand here together telling you their stories about being Samoans living with diabetes, their intention is to intensify invaluable knowledge, that is destined for future practices when working with a vulnerable population like Pacific people. The participants' experiences put together provides you, the reader, with essential insights about their realities, that assists in generating knowledge and build on health awareness relative to Pacific people living with chronic illnesses such as 
diabetes. For these reasons, I remained strong in my belief that their togetherness and specialness as Samoan people with diabetes, tenders for cohesive and meaningful insights of our life long journeys, and how they made the transition on to insulin therapy. For that special reason, here they are together, telling you their story.

\section{My positioning of the Participants:}

I have chosen to stand alongside each of the participants, because we have an important message to tell you. The retelling of participants on how they each dealt with their own realities with Type 2 diabetes and what their experiences were when transitioning to insulin therapy. Their narratives have unpacked, added meaning and provided insights into their world, as Samoan individuals living with Type 2 diabetes in New Zealand. I chose to present my findings this way because I am a strong believer that although our stories are unique and exclusive to the individual; however, our point of view is made clearer when we stand together as one. As we come together, our voice is louder and our message is patent. You will begin to see that as each theme unfolds, their narratives will inform you of their experiences, and you begin to get a sense and feel of the realities they encountered as Samoan people living with diabetes; how they lived with the challenges that came their way; and how they made the transition on to insulin therapy. We hope to provide some insights relative to the health of Pacific people in New Zealand, and how they live with chronic illnesses such as Type 2 diabetes.

This is how I endeavour to set out the outline for this chapter. First, I introduce each of the four participants with relative information of their environment and some background details when they were initially diagnosed with Type 2 diabetes. Following that, is my analysis of the findings with the use of narratives in an orderly manner. From there, you begin to experience their diverse encounters with diabetes; how each of them dealt with own realities; the challenges they encountered, and how they each made their transition on to insulin therapy. The journey alongside the participants, provides light, and brings to the fore the meaning to the themes extracted in their worldview. To conclude the chapter, I present a brief summary of the findings in light to the themes extracted. 
The participants are Sina, Sione, Emma and Pua (pseudonyms). First, I would like you to meet Sina.

\section{The Participants:}

Introducing Sina

Sina was born and raised in Samoa. She and her aiga (family) came to New Zealand about six years ago in search of better education for her children and employment opportunities. Prior to resettling in New Zealand, Sina described herself as an "active person who worked hard to achieve the best for her family" because she didn't want her family to be deprived of any necessities. Sina is a caring wife and a loving mother and grandmother of three children and four grandchildren who all live nearby. Sina remains active in her community, with considerable involvement in her local church and reciprocates help and support to her wider aiga both here in New Zealand and Samoa.

At first, Sina initially found her new life in New Zealand a little difficult to adjust to, but over time, she has found comfort and reassurance in the presence and support of her family and extended families. Not long after arriving in New Zealand, Sina was diagnosed with Type 2 diabetes, after her acute presentation to the hospital with recurrent boils that were a challenge to heal, her sudden weight loss and polydipsia. To her disbelief, she was told at this presentation she has diabetes. Sina was only 46 years of age at the time.

\section{Introducing Sione}

Sione was born in New Zealand to a Maori mother and Samoan father. Sione admits that it is his Samoan side of the family that is the dominant of his cultural identity. He recalled being raised within his wider Samoan extended aiga, where he enjoyed lots of real Samoan meals such as taro, lu'au (taro leaves in coconut cream) and corned beef to name a few, at the many gatherings held in his lifetime that he could remember as he reminisced of them with a humble grin.

Sione is a loving husband and father to his four children. He and his family are very close, and he enjoys every minute of being a grandfather to his only granddaughter whenever he 
can. He has an outgoing personality and is respected by his colleagues at his job where he is employed fulltime. Sione described his initial confrontation with diabetes as a "near death experience," because of how gravely unwell he was. He remembered his health failing but kept working and ignoring the obvious symptoms such as fatigue, polydipsia, polyuria and weight loss. It wasn't until he couldn't get up one morning for work that he realised just how severe his condition had become. Sione was sent to the hospital via his family doctor. He was 44 at the time of his diagnosis.

\section{Introducing Emma}

Emma was born and raised in Samoa, but came to New Zealand in 1992 when she was a young woman in her 20s. She too immigrated to New Zealand in search for a better future hoping to find employment so she could financially support her parents and families back home. Emma found life, in her new environment, very different from what she was used to and allowed herself time to grow to know her new home. She also found that although she was far from her homeland of Samoa, she continued to attend church and kept close ties with her family members and relatives here. Emma is now married with two sons, and has continued to keep close ties with her parents and her whole aiga back in Samoa.

Emma's journey with diabetes began when she felt acutely unwell with lethargy and tiredness. She remembered feeling it for some time, but thought nothing of it blaming it on the flu symptoms and working too hard. She didn't see the need to consult her general practitioner (GP) until her symptoms began to bother and had impact on her daily activities. In this GP visit, a full health check was carried out including a laboratory test. The laboratory results showed Emma had diabetes. This was how she found out she had developed type 2 diabetes. Emma was only thirty five years old at the time she was diagnosed.

\section{Introducing Pua}

Pua came from Samoa in 1997 with her husband and their two children in the hope of making New Zealand their new home, and to take advantage of employment and education opportunities. She graduated from University almost 10 years ago as a mature student with 
a degree and now works fulltime. Pua takes pride in her work and is very active within her local community and church.

Pua is a proud mother of five children - three of whom she conceived in New Zealand. Of her three pregnancies in New Zealand, Pua developed gestational diabetes during two of them. She was warned of her predisposition to diabetes later in life, which subsequently occurred. Pua was diagnosed about six years ago by her GP following a symptomatic episode of polyuria and increased thirst. Pua carried out a full blood test which confirmed her diabetes.

The next section discusses the first main theme, which is living with diabetes. It will be explored through the subthemes; reality, struggles and family life. The participants shared their stories. Their experiences unraveled their realities, their struggles and the evolving changes within their families. I will also be drawing on the occasional literature to introduce a finding, and assist with the exegesis of the narratives.

\section{Living with diabetes}

People diagnosed with diabetes can find the circumstances overwhelming. Given that diabetes is life long, people with diabetes can struggle to come to terms with their diagnosis. A qualitative study by Kelly, Lawrence and Dodds (2005) on women's developmental experience of living with Type 1 diabetes, revealed the women's accounts of struggle for equilibrium in a number of ways, including a disruption of stability in their lives.

In this research, each of the participants described what living with Type 2 diabetes has been like for them, from the time they were diagnosed. Their exclusive and exceptional experiences recaptured the realities on what living with diabetes entailed. Strikingly enough, a lot of prominent similarities were embedded in their stories. Their recollections of the hurdles they confronted as they carried on living with diabetes were incredible. They spoke of how they coped with their diagnoses, and their subsequent transition onto insulin therapy. These accounts unraveled their struggles of having to modify their eating practices 
of the Pacific foods they grew up with, while at the same time, learning to become skillful at taking their medications. Many changes were arising within their families, and as a consequence, these impacted and shaped the participants' realities of living with type 2 diabetes. We have no idea on how to live and be joyful in the community, with a chronic illness like diabetes.

Sione struggled following his diagnosis because of a lack of knowledge about the disease. Here he talks about not knowing much about diabetes at all when he was initially told he had it.

When they diagnosed me having diabetes I didn't have a hell lot

of an idea what it is. I did know that it was a disease and pretty

much was to do with the sugars and that's all I did know, not

much after that. (Sione, p.2)

Living with a chronic illness like diabetes is challenging. It is challenging because many people who are diagnosed with it, are not prepared to deal with its complexities, and the impact it can have to their lives and the lives of their loved ones, are overwhelming (Funnell, 2007). Challenges such as changes in lifestyle and dietary intake can cause all sorts of difficulties and disappointments for the person concern.

Take for instance the cultural foods Sina grew up with all her life in Samoa. Sina talked about her love for her traditional Samoan food, and admitted to feeling unhappy that she had to make changes to a newly adapted lifestyle following her diagnosis. In due course, she realised why she had to make these crucial changes. Sina took charge of her lifestyle changes in an effort to achieve better diabetes control. But it was not an easy expedition. She described her inner resistance feeling to food as the cause of her losing control at times. She would end up satisfying her needs by eating the food she knew she isn't suppose to have, knowing very well that her glucose reading would be very high later on. This phenomenon of making changes and adjustments to traditional and familiar lifestyles can 
be devastating.

Ia o le mea muamua a, sa lé fiafia...o mea sa masagi ai ga 'ai ma igu ai, ia a o lea ua fa'asà mai...pei la o le lé fiafia lega e i’u a iga kago aku solitulafono...i le soli lea o le tulafono, ua maukigoa o le a alu foi i luga le suka. (The first issue, I was unhappy...things I used to eat and drink I now cannot have... so because of that unhappiness which results in breaking the rules, knowing very well that by doing so, glucose readings will be high). (Sina, p.6)

To be told you have diabetes is overwhelming - another critical aspect to acknowledge when living with a long term health issue such as diabetes. Many factors like the individual's perception, and previous knowledge about diabetes, can all determine how the news about having diabetes can be received. Emma shares with us her personal description of what she feels diabetes is about. She described diabetes as a disease similar to that of any form of cancers. She goes on to enlighten us on how she had to get used to taking medications to keep her glucose levels down.

Ouke kalikogu e leai a se 'ese'esega ma ma'i foi ia ma le disease foi ia o le cancer i lo'u kalikoguga...o au o se kagaka ouke fefe ouke igu fuala'au, a e o le mea lea ua i ai gei ua igu a oga o le gaugau iga ia fa'aikiikia le suka i lo'u kigo. (I feel that diabetes is no different from cancer...I am someone who is frightened to take pills but now I'm having to take them because I am keen to keep my glucose down). (Emma, p.3)

Leading on from her description of how she viewed diabetes, Emma, in a similar situation as Sione, remembered not knowing much at all about diabetes when she was initially diagnosed. She recalled being given medications and being told to take them. Emma admitted to not taking her medications regularly, often because she had no understanding 
of the importance of doing this.

Mai i le amakaga, e le'i kelè so'u malamalama i ai i le suka; ga o le pau a le mea ga ou alu aku ai ma fa'amalamalama mai o a'u ua suka. Ia aumai fuala'au ma mea i lega vaikaimi - ia ga oka sau à lea ma ka ika fuala'au i le igu. Ia, 'aka le fia igna a la, ua ka'akiakia a lea i'i i le fale oga o le kele o aso e lè igua. (From the beginning, I didn't know much about diabetes, all I was told was that I have diabetes. Then I was given medications - which I then brought them and started to take them. I just left them here in the house, because for many days I don't take them). (Emma, p.3)

The participants' experiences with diabetes have been overwhelming. Their encounters are the proven evidence to that. We have been informed of their tremendous grief when they were first diagnosed with diabetes, and we catch a glimpse of their immeasurable struggles with adjusting the pacific foods they grew up with. We are only just starting to find out what really happens to Pacific people with Type 2 diabetes. The next subsection delves in to their reality.

\section{The reality}

Realising that you are living with a life-long chronic health problem is the reality of those who have been diagnosed with an illness such as type 2 diabetes. It is the unfortunate reality for all the participants interviewed. Each of them revealed individual thoughts and feelings about their diagnosis and how they each dealt with that realisation independently. Mixed emotions are spoken of, painting the picture of what that reality was like for them.

Of the four participants, three reacted very similarly when they were initially told they had diabetes. These reactions were predominantly negative, and included feeling overwhelmed and they talked of struggling to accept their diagnoses. The reality of coming to terms with a new diagnosis, such as diabetes, can only be expressed and felt by those concerned, as only they are aware of these inner feelings. Emma shared her reality when she learnt that 
she developed type 2 diabetes.

Sa 'ou fefe ma 'ou popole foi lele a 'ou mana i lea ma'i ia ua o se ma'i faigaka e léfaigofie. Ua kaofi uma ai mea ka ke maga'o ai ma mea sa oka fiafia ai ma pei o le a kaofia uma ai oga o legei ma'i. (I was scared and worried that if I have this health problem, it's going to be difficult and it won't be easy. I would have to stop the things I enjoyed doing all because of this health problem). (Emma, p.2)

For Sina, she too was confronted with her own reality - her reality with diabetes. She expressed feelings of grief and sadness, when realising the inevitability of diabetes. She used a metaphor to explain these feelings. Sina described having diabetes as "a thing or something" that has taken away some of her strength. She also spoke about not having the feeling of complete happiness any more, since she was diagnosed.

Ia ae ina ua ou ma'i, ua ou iloa ua tele foi le suiga... e pei ua aveeseina atu le isi vaega o le malosi....ua ou iloa à e mo'i o lea oute malosi auā o lea oute faigaluega, a e ua lè tutusa e pei o le mea sa i ai...e o'o foi i lo'u fiafia, e lè o atoa le fiafia. (So when I became sick, I knew there were many changes within myself...it's as if some of my strength is taken away....I realise that although I may still be strong because I go to work, but it is not the same anymore as before... and even my happiness, my happiness is not complete). (Sina, p.4, 5)

Sione on the other hand, explained how he took his chances in life right away by embracing his reality with diabetes, the moment he was told he had it. Sione made a conscious rationalisation connecting his crippling health to his undiagnosed diabetes. Perhaps in a blameless way, he was relieved to be given an answer to his immense suffering. It was through this experience of unwellness resulting in an acute presentation at the hospital that gave him the chance to realistically see things differently, and most of all, 
his acceptance of his diagnosis.

I accepted it more or less straight away because at that time I knew there was something wrong and when they told me I had it I said well maybe probably why I felt like this. (Sione, p.2)

Developing diabetes is the reality of the one with it. The participants have told us theirs and what mind blowing insights they were. It makes us realise just how complex that initial diagnosis can be. This next subsection discusses the challenges and struggles the participants had to confront. Their accounts expose significant information, highlighting how each of them coped and dealt with diabetes and the events they endured.

\section{The struggles}

The realities of living with any chronic health issue can impose immense daily challenges for those affected. Creating life long changes can be a challenge because of the need to adapt to healthy choices, and make healthy decisions that will benefit their outlook in life. For some, these challenges are endless, and much needed changes can take time to occur during the initial time of diagnosis.

The participants all talked about the diverse challenges they encountered in their journey with diabetes. Each of them described what some of these encounters were like for them, depicting the suffering they each faced along the way. One of the common challenges among the participants in their revelations, related to their adjustments to the traditional and cultural foods. This was one major hurdle that the participants commonly shared. They talked about their love for the foods they grew up with, and how difficult it really was to transform to the choices of food they were informed of. They then instantly realised that this journey with diabetes wasn't going to be a smooth ride.

In Sione's story, he stated that changing his eating habits was a real challenge for him. He genuinely talked about being brought up eating the island food that he loved, such as pork and taro with coconut cream. But to be told this kind of meal had to change to suit his 
diabetes needs, was really hard to grasp.

The biggest challenge for me was eating. That's a change because you've been brought up a certain way doing your food....you get your food that you've grown up with for so long, all your heavy meals, your island food like your piece of pork and taro topped with coconut cream. (Sione, p.2, 7)

Sina also added that it was difficult for her to take on the concept about changing her food, and eating choices from the foods she was accustomed to. Sina referred to the Island food she loved. She spoke metaphorically and used the symbol of "the fence" to illustrate what this change was to her. Sina's analogy of the fence, suggested a boundary enclosed within a small space, portraying her limitation of food choices. She found this change to be frustrating.

O mea sa masani a'i ona 'ai! Ia, o la e naunau i ai, a e e pei o lea ua fai e le fomai le pa e ke alu aku ma gaka ai. (Foods people used to eat! I am still keen to have them, but it's as if the doctor has now set a fence for you to stop at). (Sina, p.5)

Growing up knowing that food is accessible, it never crossed Emma's mind that she could one day have to watch what she ate, and be thoughtful about how she cooks her food because she would develop diabetes. Her experience in changing her old habits on food selections and preparations, also built on the notion about the challenges she faced when she was told she had diabetes. She recalled eating any types of food freely before and how much she loved the food she grew up with, which in this case, are the island food. But since her diagnosis, she really had to watch what she eats, and how she prepares her meals now.

Ia ke au ia ma 'ou lagona ā ia ga ala ai oga 'ou faiaku e faigaka, oga auā foi la i le mea ga oka ola mai ai, sa free laka 'ai i so'o se 
mea'ai...oga ua iloa gei ua ma'i suka, ua fuafua le 'ai, ia ua fuafua foi le faiga o mea'ai. (To me, I think that the reason this health problem is difficult is because I grew up free to take any food...but now knowing that I have diabetes, I now manage the food I take and also watch how I prepare food). (Emma, p.2)

Confronting the challenge to changing from the traditional and cultural foods was not the only critical factor for the participants to overcome. Simultaneously, there was also the great challenge of processing that they have a life-long chronic health problem to live with. The participants talked about their negligence towards their illness. They spoke about their instantaneous feelings of disbelief and denial. They were astonished to learn that even in their early forties, which is considerably young, diabetes exists. These feelings and beliefs added to their own sceptism and doubts, given that they know neither their parents nor close family members had diabetes.

Sina relayed how she lightly took the news she had developed diabetes. She refused to believe her doctor, and even wondered whether he could be lying. In her mind, she should not have diabetes because no one in her family has it - no one except for her now.

E le'o mamafa i ai le mafaufau i le tulaga o le ma'i suka...sa fa'asolo lou mafaufau i totonu o lo'n aiga e leai se isi e ma'i suka...sa 'ou mafaufau pe pepeplo le fomai oute lè suka. (I do not think seriously about diabetes...I thought of the history of my family and realised that no one has diabetes...I thought, maybe the doctor is lying and that I don't have diabetes). (Sina, p.2)

Similarly, Emma found it extremely difficult to accept she had diabetes. When she left Samoa, she never heard about her parents having diabetes; therefore, she was convinced that she couldn't have developed it - not at her age. It was not easy for Emma to understand and believe that sure enough, she has diabetes. She even thought that diabetes was only a short term health problem; that it would go away in due time. These were some 
real challenges Emma faced whilst living with diabetes.

I le kaimi mиатиа a fa'ako'a iloa ai ua ou mana i le suka, ia ga faigaka foi gale ga ou kalikoguiga ouke suka, oga foi la lea sa ou sau mai Samoa e le'i fa'apea ga ou iloa ga ma'i suka gi o'u makua ...o le mea la ga sa faigaka ai foi oga ou kaliaiga o au ouke suka. Sa i ai foi a lo'u mafaufau ma ou lagona o le suka foi a e lè kumau e alu alu ke'a. (When I first learnt I had diabetes, I didn't believe it, because when I came from Samoa I knew that none of my parents had diabetes ...that was the reason why I found it really difficult to accept I was diabetic. I also thought diabetes was for a short period, it's not permanent, and that it goes away in due time). (Emma, p.1)

The revelations of their significant struggles are believable. It serves as a reminder that adjusting to a chronic illness equates to great distress. The participants are stunned about having diabetes, and to have to adjust the foods they grew up with, added to their suffering. The participants disclosed the changes that occurred in their families; we look at these changes in the next subsection.

\section{Family Life}

The participants spoke of, and make reference to the experiences of their families when they learnt about their diagnosis of diabetes. They go on to acknowledge and value the contributions their family members had put in to support this life long journey. One of the life changing beliefs shared by the participants, is the indispensable value in living longer to see their children and grandchildren grow up. These revelations clearly inform us that changes in their family lives have emerged. Other adjustments include making positive changes together as a family, and offering support to ensure positive progresses are sustained.

Sione spoke of his family and how they managed some of the lifestyle changes they endured together. This togetherness helped ensured Sione that he could count on his family 
for ongoing support.

They are very aware of my situation my immediate family, and they also know the importance of like a good diet.....too much sugar and even too much fatty stuff as well....they're very aware of it and they too now have changed. I insisted on that because what we eat they have to eat and it's all changed now. (Sione, p.8)

The support from the families can go a long way, but there is also the crucial need for the individual with diabetes to need to help themselves to achieve good diabetes control. In Sina's case, she realised what she had to do to stay well so she could enjoy more time with her family. She told of the revelation about how important it is for her to acknowledge, value and follow the advice from the health professionals. Sina came to the realisation that for her to be around longer with her family, and cherish happy memories, she needs to ensure she looks after herself and sustain good diabetes control. Here is what she had to say about it:

E fa'ataūaina ia te au lo'u va'ava'aia lelei o lo'u suka ona o lo'u ola. E telè lo'u àfaina pe afai e lè va'ava'aia lelei e a'u lo'u lava suka...Pei la o lo'u iloa, a 'ou usiusita'i $i$ vaega ia e pei ona talanoa $i$ ai, o le a telè foi se isi taimi oute ola ai ma matou mafuta ai ma la'u fanau ma lo'u aiga. (It's important for me to maintain good diabetes control because my life depends on it. If I don't take ownership of my diabetes, I can get in to serious health problems...I realise that if I keep good control, I can live longer and be around my children and family). (Sina, p.8)

Sione shared a very similar view to Sina, in that one's health is one's responsibility too. Obviously if diabetes is not managed well, there are life threatening consequences for the person with diabetes. Should they pass away because of complications, this can cause great emotional stress and strain on the whole aiga. 
No one else can help you but yourself and if you, if you blatantly

ignore it there is only one place for you to go and that's six feet under.

That's what will happen eventually and on top of that not only do you cause trouble for yourself you cause trouble for your family because they don't need that. (Sione, p.7)

On the other hand, for Emma, shortly after she was diagnosed, she initially felt that her family was not showing support for her in this journey with diabetes. She felt that her family was not always helpful and that they did not always bother to help out. But when Emma began to experience hypoglycaemia episodes that triggered her family to become concerned and took notice of her condition. Emma's families were now more involved with her diabetes management. Her children assist with her injections and also make sure she is reminded to take her medications regularly. Emma openly talked about the growing connectedness within her family, between her, her husband and her children around her diabetes.

E o'o foi i lo'u aiga sa lē fesoasoani mai ia te a'u i le amakaga iga ua iloa; sa le ago foi ia lakou. A o le kaimi legei, a o'o iga oso lo’u suka ua kekeke ou kigo ma mea fa'apega - pei la o mea fa'apega ua amaka ai oga amaga'ia e le makou aiga ma la'u fagau lo'u suka lea ua makou galulue fa'akasi ai i le kaimi legei. E o'o foi la i le faiga o lo'u kui, ua iloa uma e si 'au fagau i le sui le vaila'au ma kaugapa mai ia ke au pe ua fai lo'u kui i le kaeao ma le afiafi...e kake lagoga ai foi loka fiafia i le makou aiga ma si au fagau o la e amaga 'ia lo'u suka. (Even my family didn't use to help me when they first knew about it. But now, when I get a low sugar, my body shakes - that's why now my husband and children are beginning to acknowledge my diabetes and we now work together. Like my insulin injection, all my kids know how to change the cartridge and constantly remind me of my insulin in the morning and in the evening....I feel very happy knowing that my husband and children support me and my diabetes). (Emma, p.12,13) 
Family support plays a fundamental contribution in Pua's life with diabetes as well. She relays how her husband assists her with her insulin injections. He makes sure that Pua is reminded of her medication so she doesn't miss them. His support helps improve her management, but also encourages a positive attitude.

\footnotetext{
Pe na o le matou aiga lea e iloa le faiga o lo'u tui...O isi foi taimi e faimai e “manatua”, e fa'amanatu mai ia fai lo'u tui, po'o ua inu la 'u fuala'au. (It's just my husband who knows how to do my injection... other times he tells me to "remember", he reminds me to do my injection, or take my tablets). (Pua, p.9)
}

The changes that took place for the participants and their families have been encouraging, in that it highlights working together despite the complexities. One other important issue the participants discussed was the notion of taking ownership of diabetes. It made them realise, that in doing so, enables them to live longer and be joyful with their families.

The next section focuses on the participants' transitions on to insulin therapy. The subthemes: reality, challenges and turning points, intensify and deepen these experiences. The participants tell us some fascinating accounts on their experiences.

\section{Making the transition onto insulin therapy}

Many scientific researchers have proven the long term benefits of insulin for those who are successfully using it. Large studies such as the DCCT (1993) and UKPDS (1998) proved this. Such advantages include improved diabetes management and better quality of life ahead. The early use and uptake of insulin is pivotal to limiting complication progression over time, which often means an improved quality of life. In spite of these known gains, often the introduction, or indeed even the mention of insulin therapy, is never easily received by the people desperately needing it (Bogatean and Hâncu, 2004).

Often the discussions on the use of insulin with those concerned, are sensitively driven with empathy and compassion, hence, enhance the necessity of it. Mathews, Peden and 
Rowles (2009) highlighted in their findings the invaluable place for effective communication. They maintained for healthcare providers to be empathic with their patients when discussing insulin. These concerns highlight the great challenges placed upon the patients, on the phenomenon of transitioning onto insulin therapy. The participants revealed their beliefs and how they felt about insulin. They also make reference to how they reacted, when they were told about the need to use insulin. The journey has not been easy. They each described their struggles, the fears and the predetermined ideas they encountered about using insulin.

When Sina was first told about the need for her to use insulin, she initially felt scared, and instantly refused to ponder the idea of using it. There were questions in her mind about what might happen if she ever missed her injections. In addition, she had doubts and was uncertain about using insulin. Sina conveyed what she encountered.

Ina ua fai mai le foma'i o le a fai le tui, o le mea muamua ā na o’o ia te a'u sa ou fefe ma 'ou musu e fai le tui...pe afai e fai le tui a e misi le tui, o le à se mea e o'o mai ia te au, po'o le à se mea e tupu? Sa fa'ailoga fesili ma e le 'o mautonu; Sa fa'alotolotolua le fa'ataunu'uina o le tui. (When the doctor said I was going to use insulin, I was frightened...and I refused to use insulin... what would happen to me if say one day I don't give it? I was indecisive and uncertain. I was in doubt about using insulin). (Sina, p. 3)

A very similar reaction also took place for Sione, because, like Sina, he too had negative perceptions and beliefs towards the use of insulin. He admitted to thinking that those who use insulin to control their diabetes are heading down the path towards death. This idea in his mind has been influenced by seeing people around him who use insulin and eventually end up on dialysis, and subsequently quickly deteriorate and pass on. Sione's reaction confirms the lack of awareness and understanding of the positive features of insulin and its usefulness. It has added to the negativity in him towards the uptake and use of insulin. 
My first impression was that if you're on insulin it was the next step to one foot in the grave you know. Well it was seeing people who are on insulin and then they're basically go onto dialysis and from there they don't last too far you know... that was probably the negative part. But not really knowing what it actually does to the body and that was the part that I was a bit unaware of and probably more afraid of. (Sione, p. 3)

Emma reacted no differently from Sina and Sione, because she too felt pessimistic about insulin. She described her struggles, and the negative feelings she encountered, the moment insulin was discussed. As a result, Emma did not accept insulin at all. She recalled developing a skin reaction while taking her oral hypoglycaemic tablets, but despite that, she was adamant to keep taking these, as long as she did not have to give in to using insulin. Emma was afraid and that meant it took her a year to consider insulin and finally get back to her family doctor about it. The influence of those close to her contributed to her rejection of insulin. She listened to their discouraging stories that implied that insulin is usually the last hope for people with diabetes. Emma's close network succeeded in swaying her away from using insulin.

Sa faigaka ia ke au oga ou faiga le kui ma ou kaliaiga le kui. Ua ou malie a e mageso lo'u kigo ma igu le fuala'au a o le tui ia e matuā leai ...sa faigaka a ga ou accept-iga i lo'u mafaufau le kui oga o a'u o se kagaka foi lele e pala'ai...e akoa le kausaga ofa'akalikali sa'u kali ' $i$ 'o i le fomai...ma o la'u faalogo i fa'amakalaga foi a isi kagaka i le kui o le-you know, that's the last hope foi lele o le kagaka o le kui. (It was difficult for me to take and accept insulin. I chose to let my skin itch and take tablets as long as it isn't insulin... it was really hard for me to accept insulin in my mind because I was afraid... it took a whole year for me to get back to my doctor... and I listened to what other people said about insulin - you know, that insulin is the last hope for people). (Emma, p. 7) 
Making the transition onto insulin therapy was no simple task for the participants. They spoke of their disbeliefs, doubts and uncertainties towards insulin. They discussed being afraid. According to them, insulin was "the last hope" and "the next step to the grave". These astonishing revelations portrayed the real challenges the participants had to overcome during their transitions. They help build knowledge on the experiences of Pacific peoples' transition onto insulin therapy.

The main theme: making the transition onto insulin therapy, will be further explored through the three subthemes: the reality, the challenges and turning points. The next subsection concentrates on the participants' realities with insulin.

\section{The reality}

For the individual who has developed diabetes, one of their greatest realities is realising that diabetes is inevitably, a progressive illness (Kelly et al., 2005). Diabetes will continue to get worse and as it progresses, the need to increase and alter treatment to achieve better control is one crucial aspect in this life long journey (Funnell, 2007). Diabetes progression is indicated by consistently elevated glucose readings or deterioration onsets in some bodily functions (such as vision, impaired sensory, hypertension) despite adhering to medications, lifestyle and physical activity. Often, oral hyperglycaemic agents are the first line medications to diabetes management (Nakar et al., 2007). But as diabetes develops, improved control and delaying complication progression can only be clinically achieved through the use of insulin therapy.

In Sina's case, she remembered when she was told about insulin by her doctor. For her, the conversation about insulin happened due to frequently abnormal diabetes blood tests, indicative of diabetes progression. Her diabetes control was getting worse and she realised the need for insulin has now come in order to stabilise her management. It was the inevitable, and only a matter of time before being confronted with using insulin for her benefit. Sina quickly realised that this was her reality and a decision by her had to be made. 
Ae o le tele à o taimi oute alu ai e va'ai le fomai pe tui foi lo'u toto, o le tala à lena a le fomai, e lè o mafai ona pa'u i lalo le suka, e maualuga pea le suka...ma ua faimai o le a fai lo'u tui. (Most of the time I go to my appointments with my doctor or have my bloods taken, the doctor always tells me that my glucose readings have not improved...and he's said to use insulin). (Sina, p. 3)

Sione's reality with diabetes, and his initial experience with the use of insulin, occurred while he was acutely unwell in hospital. He had to become accustomed to the concepts of being diabetic, and needing insulin very quickly, almost simultaneously. Sione was told about his pancreas not working causing his sugar levels to be high. He was unaware of what all this meant for him. But one thing was clear he needed to be given insulin immediately.

When they said to me I was a diabetic, because you have diabetes. And it didn't dawn onto me what that was all about... they told me that basically your blood sugar levels, you have your pancreas can't produce the insulin to breakdown your sugar and that's what's happened to you...okay, we're going to give you this...and they actually gave me insulin while I was in the hospital. (Sione, p. 12)

Emma conveyed her inner struggles with diabetes. She spoke of her emotional battle in acknowledging and understanding her health issue. She had an extremely difficult time accepting her reality with insulin and was adamant never to use it because she didn't believe in it. She doubted insulin and didn't know its clinical significance. But it wasn't until she continued taking her medications and began using insulin that helped her realise their benefits. These clinical and pharmaceutical interventions assisted with Emma's coming to terms with her diabetes and have opened her eyes to realise its seriousness and complexities. She has this to say about her chronic illness and the use of insulin. 
Ae ua avea le aumai o fuala'au ma le kui foi la lea ouke ola ai i le kaimi legei, pei ua avea à mea ga ma mea ua fa'afaigofie ai oga kaliaiga i lo'u mafaufau ma ou iloaina ai loa le leaga ma le faigaka mo le kagaka ae maua i le suka. (It is because the tablets and insulin I now use have made it a little easier for me in my mind to accept and realise how complicated and difficult things can get for the person with diabetes). (Emma, p. 5)

Having spoken of the inevitable transition to insulin therapy, insights of their realities are unpacked. These include the need to consider insulin because diabetes management was not achievable. For Sione he was more or less given insulin during his admission to help with his recovery. No matter the circumstances, the participants faced up to the realities of needing insulin for better diabetes control.

The next subsection discusses the challenges that got in the way for the participants. Let us hear their stories of the issues they contested.

\section{The challenges}

Making the rightful choice to transition onto insulin is never a simple step to take for the individual with diabetes. This is because of the obstacles that counter the positive clinical impact of using insulin therapy; however, many challenges stand in the way. These often succeed in swaying those in desperate need for such intervention towards the wrong directions, and as a consequence, can result in serious secondary complications (Bogatean \& Hâncu, 2004). The participants revealed the challenges they each encountered. In their experiences, these challenges made them doubt the clinical advantage of insulin, and therefore, caused inner contentions and refusal.

Sina and Emma tell us of their experiences with the challenges that came their way. They faced up to similar issues brought on by those close to them. People in their family circle, as well as close friends, gave false information about insulin in an attempt to influence their decisions against using insulin. The participants had been told that "insulin is not good" and it is a bad thing to be taking. These false statements did not help Sina and 
Emma's decision to use insulin and also influenced their decision making. This was Sina's encounter.

E tele le fete'ena'iga i le va o a'u ma a'u uo, ma tagata o lo'u aiga...o la foi e fa'alogologo latou i isi tagata ofa'apea mai: "e leaga le mea lena o le tui” omai loa tagata o lo'u aiga la ua iloa o a'u lea o le a faia le tui, na lā ua omai ua fa'apea mai: "e leaga le tui". A e a 'ou fesili atu: "E fai sou tui?" ae faimai: "E leai". Ia ae o tatou e tata'igofie à le mafaufau i le isi tagata. (There is a lot of tension between me, my friends and members of my family...they too are hearing other people say: "insulin is bad" they then came to me and said: "insulin is not good". But when I ask: "Do you use insulin?" and they answer: "No". I guess we let others mislead our minds so easily). (Sina, p. 12)

Emma was given a slightly different message on using insulin. She shared that:

O kagaka sa kalagoa mai ia ke au, sa ou mana ai foi gi fa'amakalaga; ia o kagaka à o au friends ia ma kagaka o lo'u aiga sa kalagoa mai ia ke au e uiga i le kui...ga faimai à ia ke au ouke kaumafai e 'aua gei ou o'o i le kui oga faimai e faigaka pe a o'o i ai le kagaka. (There were people that spoke to me, whom I received information from; people such as my friends and family members who talked to me about insulin...they told me to try and not use insulin because it is dangerous when the person gets to that phase. (Emma, p. 8)

The responsibilities required for administering insulin can also build on to the challenge and give rise to the rejection to using insulin by the ones concerned. Injecting insulin occurs daily, and it needs regular monitoring and management. This can be overwhelming and takes time to get used to. In Sione's experience, he talked about the challenge he confronted: as he was faced with actually giving the injection, in which he described as 
"the negative part".

The negative part about it was jabbing you with the needle you know to administer the insulin... if you know your sugar levels are too high then you know obviously you are doing something wrong. (Sione, p. 4)

The participants spoke of the challenges during their transition to insulin. People around them had a part to play, in giving inaccurate and misleading information about insulin. Moreover, injecting insulin was perceived as negative because it involves injecting oneself. These experiences reported by the participants, which added to their challenges, and hindered a successful transition to insulin therapy. In the next subsection, the subtheme: turning points will be described.

\section{Turning points}

Changes in peoples' lives can take a long time to occur. For some, changes to their health habits and beliefs may never arise, which impede improvement and progress of their diabetes (Funnel, 2007). Many years of living with diabetes doesn't always mean that there is an intention for the person living with it to take ownership and total control of their chronic health problem. In some unfortunate cases, it may take for some serious medical issue, or an acute hospital admission, for the concerned individual to start contemplating their health and the risks they are taking due to not managing their diabetes for so long. Often, such experience can spark a change in the way health should be owned and managed by that person. The participants in this study all had an eye opening incident that changed their perspective. They inform us of how their respective experiences were a turning point for them.

Sina learnt a lesson the hard way through a hospital admission when she was acutely unwell. This happened because she stopped all her medications including insulin. She withheld her treatments because she was half-hearted about them and was in great doubt about their efficacy. Sina remembered how very worried she felt at the time. She blamed 
no one else but herself. She has clear memories of how gravely ill she felt all because she refused to listen and take on board advice for her health. It was the course of this acute experience that prompted her to relook her decisions related to her health and decided to change. This was the turning point for Sina. She revealed how pivotal it was for her to take ownership and show interest in her own health - that she is key to staying well in managing her diabetes.

Oute le'i toe faia se tui, oute le 'i toe inuina se fuala'au. Lea la ua o'o i le taimi ua o'o mai le tigaina ia te a'u...na o'o mai ia te a'u le popole ma sa 'ou mafaufau ona o a'u lea ua āla ai...ona o a'u i lo'u lè usiusitai... sa 'ou fa'atalatalanoa! sa le'i manatu mamafa i ai! sa fa'alotolotolua! A e ina ua lagona loa le tigaina ma le tulaga na o'o i ai, lea la mai i le taimi à lena seia o'o mai i le taimi nei, lea ua 'ou iloa e tāua le usiusita'i! E tāua foi lo'u iloa ma 'ou taumafai ina ia 'ou iloa lava e a'u va'ava'ai lo'u suka. (I stopped using insulin, I stopped taking my tablets. This lead me to feeling really unwell...I became worried and it occurred to me that this was my fault... because I didn't listen...I was complacent! I thought lightly of it! I was in doubt! From that time when I became gravely unwell, until now, I have realised the value in taking onboard advice. It is vital that I also show interest and look after my own diabetes). (Sina, p. 9)

In Sione's experience, the possibility of more time in this world to spend with his children and his only grandchild was the ultimate turning point for him. During his illness at the hospital, he came close to deaths door. Sione suddenly understood just how short life can be if he didn't quickly change to healthy ways of living. He then realised that, of all the things he had, it would be of no use, because he would not be around to see his children grow up. Sione himself even admitted that it would be selfish of him to not take his diabetes management seriously.

What I look about it now is life's too short. Live life everyday as though it's your last. I got my kids to look forward to. I've got my grandchild....I'll 
put it this way; everyday above the ground is a good day....That's my goal; to watch her grow and that's why I did it. I mean not so much for her but for myself. I want to be there to watch them grow and you can't be if you don't look after yourself and at the end of the day, if I don't, that's selfish on my behalf. (Sione, p. $7 \& 10)$

For Emma, she revealed having grasped, a better understanding on the value of insulin. She had witnessed the good effects insulin has on her diabetes. She acknowledged the clinical significance of insulin in controlling her readings. This positive experience has given her the opportunity to approve and accept her continued use of insulin today. Emma saw that insulin was bringing her readings down and helped her feel better. This was the turning point for her in the way she viewed insulin. She stated that with the knowledge she now has on the benefits of insulin, she will reject any misleading messages from anyone.

Afai po'o ai e sau e faimai ia ke au a mea ia ku'u le kui; o le mea ua kakau oga ku’u le kui po’o ua lava lea kui, ia ouke leai ai la i le kala lea leaga ua 'ou iloa le aoga o le kui ia ke au ia i lo’u suka ma le mea lea ua i ai gei au i le kaimi gei-ouke lè mafai la oga ou ku'ua le kui. (If anyone comes and tells me to stop using insulin or that is enough on using it, I will say no to this notion because I am aware of the usefulness of the insulin for me and how I feel now - I cannot stop my insulin). (Emma, p. 16)

Altogether, the participant's depositions on turning points were inspired by their need to maintain good health and to live longer to be with their children. These fundamental aspects of their well being, depend highly on their unfailing use of insulin and taking control of their diabetes. The participants each found insulin effective in controlling their glucose readings.

The following section presents the final main theme: realisations. It will be broadened through the two subthemes: seeing is believing, and what insulin means. 


\section{Realisation}

People living with diabetes often struggle to acknowledge that diabetes is a life long chronic health problem that progresses over time (Brown et al., 2007). Diabetes is the cause of serious secondary complications such as kidney and heart disease and blindness to name a few. To learn of these serious effects caused by diabetes is frightening. On the positive note, such awareness allows the person with diabetes to take hold of what it means for them if they value and sustain stable control. For instance, this realisation and perception can aid the person with diabetes to alter their health attitudes and beliefs. The participants all share their realisations and what they went through.

Sina began to grasp that diabetes is a serious illness with real complications that will only be experienced by her. She acknowledged that her deteriorating vision is the start of secondary problems to come. At only age forty nine, her vision is slowly failing and will continue to worsen. Emma now believes the health education and information she has been provided with are true. She has come to the realisation that her fate is in her own hands. She has become aware that if she doesn't take her diabetes seriously, other parts of her body will eventually be affected as a result of poorly controlled diabetes.

E pei o o’u mata; lea la ua i ai i le taimi nei, lea la ua ou talitonu. Ia o le tele à o taimi ua lè lelei le va'ai; ae e lē o a'u o se tagata matua. Atonu o lea ua 49 nei o’'u tausaga....e tasi a le fuaiupu, lua, a ua solo'atoa ua lē mafai ona iloa. O ina la i le vaega lea, o lo’u iloa lena, afai a oute lé usita'i, o le a tele nisi vaega o lo'u tino o le a fa'aleagaina ona o le suka. (Like my eyes; I now believe. Most of the times, my eyesight is impaired; but I am not an older person. I am now 49 years old....one sentence, two, and things are blurred and I cannot see them. It is then I realise, if I don't listen, many more parts of my body will be damaged because of diabetes). (Sina, p. 7)

When Sione first learnt of his diagnosis, he didn't take it too lightly. He referred to diabetes as a sickness, which he desired to get rid of, so he could get on with life. He was 
determined to "clean it up" and "beat it" because he didn't want to accept he had a chronic illness. But what eventually became apparent for Sione, was his realisation of the nature of diabetes - that it is life long and it will never go away. He has realised that now, and despite his desire to get totally rid of diabetes; this is not going to happen. One thing he knows for sure though, diabetes can be controlled and managed for as long as he can. Sione recognised that he will never be the same person again.

My greatest desire was to beat this sickness because that's what diabetes is to me, it's a sickness and I wanted to clean it up. Get rid of it, don't want to know about it and get on with it you know. That was my biggest desire but I have now taken on board and I admit to it now that it's not going to go away. It can be controlled and that's the main thing....I'm not going to be the same as I used to be. (Sione, p. 8)

Emma was constantly lethargic and tired for no obvious reason before her uptake of insulin. She used to feel unwell and also felt lightheaded. Until the time she commenced insulin, she began to see the improvements in her glucose readings. Above all, she noticed that when her readings stabilised, she felt well and energetic. At the times, when Emma would withheld one of her injections, she noted that the ill symptoms return. These experiences lead Emma to realise how much her body needs insulin. This was a huge realisation. She appreciates insulin and how well she feels within herself when she gives it all the time.

Pei a la oga o’o mai i le kaimi gei o fai lo’u kui, o le mea foi ga ua 'ou koaga ma ou fa'amaogi ai i le faiga o lo'u kui i le kaimi legei, oga ua ōka iloa foi le aoga o le kui i lo’u suka, ma ouke malosi ai. A e 'a misi loa se aso se kasi pe lua aso, oga lagoga foi lea o le vaivai ma le 'e'eva foi lea ma le gigiva o lo'u ulu. (Up until now I'm still taking insulin, and the reason I have been consistent in taking it is because I now realise its usefulness for me as I am now well. Once its missed say for one or two days, I begin to feel tired, lethargic and lightheaded). 
(Emma, p. 10)

The clinical usefulness of insulin becomes understandable to the participants, as they embarked on their journey with it. Their realisation of its effectiveness became key as well. For instance; feelings of lethargy, lightheadedness and blurred vision recurred if insulin was missed. So, in contrast to attain feelings of wellness, regular insulin is necessary. The informants revealed how they now had their insulin regularly to maintain good health. Sione affirmed: "I'm not going to be the same person as I used to be". All these claims by the participants enhance their realisation of diabetes and clinical benefits of insulin therapy. Seeing is believing, is the subtheme that follows. It augments the realisation of the participants.

\section{Seeing is believing}

The participants discussed some interesting details on how they began believing the efficacy of the insulin they were taking. Their improved blood glucose readings as they continued taking insulin was evident. Their energy levels restored, and inner feelings of wellness, were a testimony to their improved diabetes control. Despite feeling overwhelmed about commencing insulin initially, what they experienced now since they transitioned, opened their eyes to an entirely new lease of life. These events reaffirmed in their minds that insulin helps. The informants also realised that they are central to achieving improved diabetes by making sure they look after themselves in administering insulin and taking medications. The theme "Seeing is believing", highlights attention to the health adjustments and transformations of the participants, which were a result of physical distress and acute suffering.

Sina remembered clearly how ill and miserable she felt. She was besieged with agony and pain. This was a result of pro-longed hyperglycaemia because she stopped her insulin injections. She realised that she had inflicted this on herself, and it was not caused by anyone else. She was reminded that she neglected her health. While in hospital, Sina began to believe that maintaining her health status and wellbeing rests in her own hands. Since being discharged home, Sina disclosed that she continued to take her insulin and all her 
medications. She was pleased with her positive progress and achievement, for her health since discharge. Sina was adamant to keep things that way for as long as she could. This is her testimony on her feelings of wellness, happiness and inner strength.

Na ou ma'i, na ou matua'i lagona lava le ma'i. Sa ou lagona le mafatia. Ina ua ou te'a mai ma aumai loa le talavai ma toe fa' 'amanatu mai e le fomai ia te au: "e tatau ona 'e usitai 'e inu au fuala'a; e leai se isi e te fa 'amoemoe i ai"...I le taimi nei foi, e leai ma se mea o o'u fa'alogoina i lo'u tino; oute mana à lo'u malosi ona o lea ua 'ou usiusita 'i... ua maua foi à le fiafia ina ua maua foi le malosi. (When I was sick, I felt extremely unwell. I was stricken by pain. When I was discharged home and be given my prescription, the doctor again reminded me: "you must adhere and take your medications, there is no one else to rely on but you"...Right now, I feel fine within myself, I feel well because I have complied...I am feeling happy because I am well). (Sina, p. 11)

Sione could still recall his ailment and miseries and the time he was in hospital. He remembered feeling gravely unwell. He reminisced of the acute suffering he endured, because of poorly controlled diabetes that went undetected for so long. Pondering how he now believes insulin is trustworthy as it has proved its efficacy. He is full of energy again and his good health restored. Sione admitted looking after himself since returning home. When engaged in his daily tasks, the feelings of tiredness and lethargy he once felt, have long disappeared. His revelations uncovered how he came to believe the clinical significance of insulin to his wellness.

Physically, I feel better for myself and I am...I'm thinking most of the time that I'm looking after myself and I do feel more energy, whereas prior that, I was lacking energy; yes, very lethargic...but now, I can get up carry on and do something and I'm not tired after that. Emotionally, being on insulin for me, I mean it's just another means for looking after 
myself. (Sione, p. 10)

After being misinformed and led away from using insulin for some time, Emma now praised its clinical worth in her experience. Her improved glucose readings and invigorating physical wellbeing verified this. Since taking insulin, she commended its simplicity and recounted on doing her injections with ease. Emma believes that insulin is the most preferred treatment choice for her, and evidently the most effective. She cautioned not to be deceived by other people about insulin the way she was. She used to avoid any discussions on insulin because she wasn't ready for the transition. It took her a while to realise her need of it, while at the same time she put up with feeling lethargic and fatigued. When she eventually made the transition on to insulin, she appreciated feeling well and full of life again. To strengthen her appreciation of insulin, Emma used a line from the Holy Bible to illustrate her viewpoint. Emma said: "Tom only believed when he saw". This line in the scripture refers to one of Jesus Christ's disciples called Tom. Tom refused to believe Jesus had risen from the dead, until he saw Him with his own two eyes. Therefore, in Emma's case, she only believed the benefits of insulin, when she finally agreed to take it and experienced wellness again.

Ia, e lé faigaka le kui e faigofie; auā e pei o le mea sa faigaka ai, oga fai mai isi e kiga - ia a e e leai....ma ua ou iloa foi e a'u ia le aoga o le kui ia ke a'u i lo’u lea kalikoguga; e makuā sili aku à le kui; ua sili aku loga aoga i le mea lea ua i ai gei lo’u suka; auā o lo’u suka sa maualuga, a lea ua fai ma alu i lalo oga o lo'u koaga e fai le kui... 'Aua le fa'alogo fua i le isi kagaka, o le mea taua o le alu e aumai e fa'ata'ita'i ma iloa ai foi le faigofie ma le aoga o le kui - "Ga kalikogu Koma iga ua va'ai”. (Insulin is not difficult it is simple because it was only difficult at first because of hearsay that it hurts - but that isn't true....I know it's usefulness for me and I believe insulin is the most benefit because my glucose readings have improved as I have been doing my insulin. Don't listen to other people but see it for yourself and give it a go to realise how 
easy it is to use-Tom only believed when he saw). (Emma, p. 18, 21)

The participants' testimonies on how they started believing in insulin are compelling. The clinical significance as witnessed through improvements of glucose readings, diabetes control and feelings of wellness, strengthen the subtheme - seeing is believing. Having gone through life changing events in their excerpts, what insulin means is brought to the fore. This will be analysed and reported on in the next subsection.

\section{What insulin means for me}

Insulin is an extremely effective glucose lowering treatment. Its clinical benefits have been well studied and widely reported on (DCCT, 1993; UKPDS, 1998). The informants have gone through life changing moments and acute suffering, where efficacy of insulin manifested. As a result, their beliefs and perceptions of insulin changed. Sina declared what insulin means for her. It means improved diabetes control and keeping well. She acknowledged how invaluable insulin is, necessitating commitment of the mind and soul.

Ia te au ā ia, e tatau ona fa'atāua le tui, ia ma tu'u atoa i ai le mafaufau ma le agaga....Ia te au à ia ua ou fa'atāuaina le tui....ua 'ou taliaina le fa'ā̄uāuina pea o lo'u tui mo le fa'aleleia o lo’u suka auā lo’u soifua maloloina. (Personally, insulin is essential and requires committing the mind and soul. For me, I value insulin.... I have accepted to continue using insulin to improve my diabetes for my well being and health). (Sina, p. $15,16)$

Sione noticed an improvement to his glucose readings while proceeding taking insulin. His confidence also grew as he got better at administered insulin daily. One thing is clear in his mind; that to keep glucose readings within satisfactory levels, he must accept insulin. To him, insulin means a better way of life, and a simpler way to control it.

When I first started using it, I'm still trying to get used to actually getting 
the right proportion because the first time I did it, it was only small amounts and it didn't make much of a significant change, not until probably about the third day then I noticed the change in my sugar levels were dropping due to the insulin...The more I took you know the more confident I was getting... Hopefully a better way of life. Keeping the sugar levels down to the certain right area...this is probably hopefully a better way not so much a better way of life but a better, an easier way for me to control my life. (Sione, p. 5)

Pua asserted her conscious decision to use insulin. Since her transition, her management had noticeably improved, proving significance and clinical worth of insulin. Granted, Pua's belief of insulin encompasses staying well in preparation for the future.

O’u faapea loa lea o lo’u loto, “it's okay, lea la la'u tago loa la e fai mo le lumanai ma lo'u soifua maloloina”. (I thought to myself, “it's okay; I will take insulin because of days to come and for my health"). (Pua, p. 15)

In Emma's world, insulin is the most important thing that has happened in relation to managing her illness. Despite doubting it at the start of her transition, her constant exposure and giving of insulin helped build her self-assurance and self-confidence. She witnessed the clinical significant of insulin in her diabetes control. To Emma, insulin signifies hope.

Ia ke a'u à ia i le tui, e pei oga fa'afaigaka i le amakaga, ae ua alualu à oka masagi a; lea ua faigofie ga oka faia le kui. Ia, auā o le aoga ia ia ke au à ia, oga ua suia ai le malosi o lo’u kigo, auā o le kele o kaimi sa fai ma ou ma'i; ae ia ke au, o le aoga ia o le kui ia ke a'u. O lea ua aoga auā ua o’u mana ai le malosi i kaimi uma foi ia ua 'ou lè koe ma'i ulu, ua 'ou lè koe ivā ma 'ou lē koe lèlava...i so'u kalikoguga ga o a'u i le kui, o se 
mea lea иa 'ou ola ai...o lo'u fa' 'amoemoega lea, та иа paи lea o se mea o lea ua ou kalikogu a'u ouke iloa e fesoasoagi i lo'u suka. (Personally, insulin although I found it a little difficult at the beginning, I now find it easy to use. There is a lot of benefits of the insulin for me because I used to get sick many times before, so I know how useful it is for me. I know it's usefulness because I feel well all the time, I no longer have a headache, I am not lethargic and tired...but that is my belief on the insulin, it's what I now depend on...I know I need it and I have realised that this is what is helping my diabetes). (Emma, p. 12)

\section{Conclusion}

To conclude, the unique accounts and revelations given by the participants, uncovered remarkable insights of their life-long journey with diabetes, and how they made the transition onto insulin therapy. The themes and associated subthemes, illustrated the significant suffering and the grief they experienced. These experiences created meaning, which impacted on their search for self-assurance and self-confidence, about being a diabetic. Life changing moments emerged during the journey, which have subsequently enhanced and strengthened the realisation about the perminance of diabetes. Using insulin to achieve better control was a feature of the participant's experience. Insulin, as the informants described, symbolised "hope". This is because they have experienced, and have therefore accepted the clinical impact of insulin on their bodies, and its indispensable value in achieving improved diabetes management.

The next chapter, presents the discussions of this research study findings. It encompasses details of what the study has contributed to the discipline of nursing. 


\section{CHAPTER 5: $\quad$ Discussions of the findings}

\section{Introduction}

This chapter discusses the significant overarching theme of how Samoan people living with Type 2 diabetes come to terms with their transition to insulin therapy. Embedded within the participant's stories were their realities and suffering in the journey. It is apparent that we are just beginning to realise the overwhelming grief they endured as postulated by the major themes. Therefore, to understand this better, the discussion for this research draws on the Grief Model by Kübler-Ross (1969). It was the most appropriate model to employ for it is transferable to personal and emotional changes resulting from factors other than death (Chapman, 2006), given the lack of grief literature to explain Samoan people's experience of grief. The Fonofale Model (Fuimaono, 2001), a Pacific health model, highlighted the most important values commonly shared by many Pacific people in New Zealand. The use of a Samoan fale to illustrate these Pacific values is unique and has sentimental values. For the purpose of this research, it focussed and expanded on the roof of the Samoan fale, and inextricably weave in aspects of the Grief model to better understand the experiences of the participants of their journey with Type 2 diabetes.

A model, which I called Ia Malu Model (to protect or to shelter) was created as a result of the analysis to illustrate the experiences of the participants on how they made the transition to insulin. On account of this, a description and details on Ia Malu Model will unravel the understanding that connects each phase to the participant's experiences. Furthermore, a discussion on the implications of this study for nurses/healthcare professionals will be staged as well as acknowledging its limitations. Finally, this chapter offers the conclusion for the thesis and proposes suggestions for future research that will enhance nursing knowledge sensitivity to Pacific people's experiences with diabetes.

\section{Revisit Aims and Objectives of the study}

Pacific people exhibit diabetes much younger (when compared to their European counterparts) and require insulin at a much earlier time in their young lives to achieve good control. There is virtually no research on how Samoan people living with diabetes make 
the transition to insulin. For this reason, it makes this study very valuable in exploring this phenomenon in Pacific health. The general purpose for this study is to generate knowledge and increase awareness on factors that relate to acceptability and adjustment to insulin therapy of Samoan people with Type 2 diabetes in New Zealand.

The aim of the research was to explore and describe how Samoan people living with diabetes make the transition onto insulin therapy for better glycaemic control.

The objectives of the study were:

- To learn of Samoan people's experiences, beliefs and perceptions on what it means to be insulin users,

- To learn about how Samoan people felt about commencing insulin and what they believe about the therapy,

- To learn whether they have come to terms with being insulin users. If yes, how has this happened? If not, what has been happening? To explore and uncover any personal struggles, hopes or desires that may have; during the transition onto insulin therapy and

- To discover any processes that may have helped or hindered them through these transitions.

The review of literature uncovered virtually no existing knowledge on how Samoan people with Type 2 diabetes make the transition to insulin. For this reason, qualitative descriptive research is the method of choice when there is little to no knowledge about an issue or whereby a state of knowledge is limited (Sandelowski, 2000). It demands the presentation of facts of cases in everyday language depending on the lived experience, perception, sensitivity and inclinations of the describer, with straight forward description of the phenomenon (Sandelowski, 2000). The findings from this study generated information that revealed the complexities of human responses to illness, disease and treatment, fundamental to the creation of effective and culturally sensitive interventions (Sandelowski, 2000).

This made qualitative description the most appropriate methodology to explore and 
describe the beliefs and perception of Samoan people of their experiences with transitioning to insulin through the use of in-depth interviewing.

\section{Major knowledge contribution}

Out of the analysis, emerged the generation of the "Ia Malu Model", which illustrates the experiences of the participants of their journey with diabetes and their subsequent transition to insulin therapy. The model constitutes four phases that portray these experiences; grief, realisation, hopefulness and wholeness. The fundamental concepts of knowledge transfer and knowledge synthesis are the enabling processes that assisted the participants to transition from one phase to the other.

Firstly, I want to offer a description of the fale Samoa. I will draw on my reasons to justify my choosing of the roof of the fale Samoa to create the Ia Malu model. Having adapted the Fonofale Model, the roof, out of all the parts of the Samoan fale, was the most relevant to depict the experiences of the Samoan participants' transitions to insulin. I will describe what the roof symbolises in this study to support the discussion on what it implies for nurses or HCP.

\section{Description of the Roof of the Samoan fale}

Fale is Samoan for house. There are two types of meeting or guest houses - fale tele (round house) and fale afolau (long house). They often present a very pleasing appearance especially as seen embowered in the beautiful breadfruit and coconut groves that surround them. Imagine a gigantic beehive, 35 in diameter, a 100 in circumference, and raised from the ground by a number of posts all around, at intervals of four feet from each other. That will give a good idea of the appearance of the fale Samoa. In the centre of the fale tele are two or three pillars sunk deep in the ground, and extending up to support the 'au'au (ridge pole), forming the main prop for the fale. In the space at the top is the fatuga or 'aso (rafters or listels). In between the fatuga (rafters or listels) are the 'aso'aso or pae'aso (ribs) made from breadfruit trees that are broken up in small pieces and tied together with 'afa (sennit) to form a long rod that runs from the 'au'au (ridge pole). There are a whole lot of these 'aso'aso (ribs) running from the 'au'au (ridgepole) down to the tulutulu (eaves) 
creating a cagelike frame. At the top of this cagelike frame are the thatches which are laid and tied with great care and taste. The roofs of Samoan fale are high vaulted guaranteeing maximum coolness. Their steep pitch is incredibly resistant to strong winds. "Even a superficial analysis of the unusual design of the fale reveals how successfully it satisfies several requirements" (UNESCO, 1992, p. 10).

The lau (thatches) are lifted to the outside of the roof, where they are neatly tied with sinnet one by one using the lave lau (special thatching crotchet needle made of hard wood and a great deal of sennit) on to the rafters. The thatches are laid beginning at the eaves at the bottom and working upwards towards the ridge pole. Each one overlaps one another by an inch or so, and often required 100 of them dependent on the size of the fale. This thatching if well done, can last for several years. The thatches are the only parts of the fale most likely to be replaced if they decay or wear out. There is not a single nail used to build a fale Samoa, only yards of sinnet that tie and hold all the materials of the fale together. When the thatches are all securely laid, the final part of the fale to go up to the very top of the roof is the taualuga. This is the very final phase of the fale Samoa absolutely critical for keeping the roof malu (sheltered and secured) (Krämer, 1903; Stair, 1983; Turner, 1984; UNESCO, 1992).

\section{Symbolic meaning of the Roof}

If the roof or taualuga of the fale is not built well and thatched carefully, water will leak through and the aiga cannot occupy the fale (S. Tapu, personal communication, August 16, 2010). The roof provides shelter for the whole fale and the whole aiga. It is a sign of unity and protection for life. The word "Ia" in the Samoan language is an imperative word, that when used, demands something at its final stage (N. Vaila'au, personal communication, November 6, 2010). The word "Malu" in Samoan means sheltered or protection. The concept of "Malu" portrays completeness of the whole fale. It is important to have roofs that are well malu for the security, for the safety, for the continuity and wellbeing of the whole aiga. Roofs of best houses if well built can last many years (Stair, 1983; Turner, 1984; UNESCO, 1992). The building and works essential for the roof is complicated and pose great difficulties. It's a process that takes months of hard work, involves the whole 
family (extended family), the whole village (in most cases) and the leadership and expertise of the tufuga (chief carpenter) with help from his 'au tufuga (assistants of the chief carpernter).

The roof then, in its symbolic meaning when completed, embodies the patient with diabetes who yearns for wholeness and complete wellness. If they do things well and look after themselves, they would feel well and have a sense of wholeness. The roof has imposing effects on the whole fale similar to that of the person with Type 2 diabetes who can impose on their families. If the tauluaga (roof) is leaking, the aiga is not malu (safe). If the person with Type 2 diabetes stops taking their insulin and medications, they will be unwell, and will affect their relationships with their families, community and other people (N. Vailaau, personal communication, November 6, 2010). The cagelike frame consisting of the 'au'au (ridge pole), 'aso (rafters or listels), 'aso'aso or pae'aso (eaves or listels) symbolises the multiple roles of the nurse/health care professional with its intertwining and interlinking parts, that support and enable the patient with diabetes to maintain a normal life, and helps them cope with the burdens of their illness. The cagelike framing for the taualuga (roof) has been constructed in a manner that when thatches are laid, they can only be done from the bottom up.

The gathering of the leaves to make the thatches signifies the gradual build up of knowledge and understanding of the patient of their illness. The thatches represent the patient's knowledge; fragile and exposed to the elements. When the thatches are placed on the roof, they are positioned one thatch at a time to ensure proper placement and secured tying with sinnet to the listels. Each thatch overlaps one another starting from the bottom, one row at a time and so on all around the house towards the ridge pole at the top. Like the patient, as their knowledge expands and grows with the help and directions of the caring nurse, their confidence will slowly establish as they take strides one step at a time towards wholeness and feeling well.

Thatches when decayed will require replacement. Like the patient's knowledge, it is always evolving and changing (for good or bad), and when the interactions and 
communication between the nurse/health care professionals and the patient is grounded on mutual sensitivities, better outcomes and improved health choices and diabetes management follow. The chief tufuga denotes the nurse, who leads the patient through their suffering towards recovery. The tufuga (chief carpenter) leads the building of the fale, similar to the nurse who is always visible and "present" for the patient. Nurses need to be caring, compassionate, and culturally sensitive with competent qualities that uphold connectedness with the patient. Benner (1984) pointed out that "sometimes the experiences of a patient are extremely foreign to the nurse...but understanding come by letting patients teach the nurse what it is like to be in their shoes" (p. 68). Nurses are the cultural bearers (Ramsden, 1994), and whom the patients turn to in their times of need (Benner, 1984).

The next section describes the Grief Model and justifies why its principles are the most appropriate for conceptualisation of this study.

\section{The Grief Model}

I have chosen the Grief Model (Kübler - Ross, 1969) on the merits of the direction it offers in providing insight, and guidance for coming to terms with personal trauma and emotional adjustments. There is no work done that I could locate to resonate for me, both as a diabetes clinician and a Samoan to best understand grief in the same way Kübler - Ross (1969) describes it. The Grief Model offers a deeper understanding of grief and it is the best way to approach it in relations to what I have seen. The principles and concepts of the Grief Model foreground the experiences of Pacific people with diabetes and of their transition to insulin therapy.

The Grief Model (Kübler-Ross, 1969) was the most appropriate model to employ because it is transferable to personal and emotional changes resulting from factors other than death (Chapman, 2009). In light of my clinical experience, what Kübler-Ross (1969) describes in her pioneer work, is very comparable to the phases observed in Pacific people who have diabetes. The people experience grief in their journey. While death and dying are for many people the ultimate trauma, people can encounter similar emotional upsets when 
confronted with life challenges, in particular something difficult for the first time. Trauma and emotional shock are relative terms of effect on people (Love, 2007). Grieving among the participants was a relative emotional trauma. This seminal work by Elizabeth Kübler Ross (1969) which aimed at providing support and counselling of personal trauma, grief and grieving affiliated with death and dying. Her positive influences through her pioneering work significantly bettered the understanding and practices that relate to bereavement and terminal care. What was significant about her work was recognising that individuals have to pass through their own individual journey of coming to terms with their reality. The five stages of the Grief Mode are: denial, anger, bargaining, depression and acceptance. Kübler Ross (1969) not only proposed the five stages of grief, she also threaded in "hope". She maintained that hope was visible in all stages of grief, and that a glimpse of hope enabled people to endure strains and rationalise suffering. These are the concepts of the Grief Model:

Denial: - this phase relates to a conscious or unconscious refusal to accept facts, information and reality, related to the relative situation. Some people can get stuck in this stage when dealing with a traumatic change that can be ignored. Death in this case is not particularly easy to avoid or evade indefinitely.

Anger: - If the first stage of denial cannot be maintained any longer, it is replaced by feelings of anger, rage, envy and resentment. Individuals dealing with emotional upset can feel angry with themselves or others, in particular to the people closer to them.

Bargaining: - This stage is less known but equally helpful for the people facing death. This stage can involve attempting to enter in to some sort of agreement/bargain with whatever God the person believes in.

Depression: - Also referred to as preparatory grief. The person finally finds acceptance with emotional attachment. Often there is no or little need for words, with feelings such as sadness and fear. This indicates that the person has at least begun to accept the inevitable. 
Acceptance: - This stage varies depending on the person's situation. It is almost void of feelings as if the pain had gone and the struggle is over. People dying enter this stage well before their families and the ones they leave behind, who necessarily pass through their own individual stages of dealing with grief.

Hope: - This stage persists throughout the five different stages of grief when people are faced with tragic news. It is a defensive and coping mechanism to help people deal with extremely difficult times. It is hope that enable to endure strains and rationalise suffering.

The Grief Model is the most suited framework to theorising the stories of the participants. The five stages in association with "hope" provide light to the individual journeys of coming to terms with reality of diabetes and making the transition to insulin of the participants. The next section discusses the phases of the Ia Malu model in light of the concepts of the Grief Model. An illustration of the model follows after the discussion of each phase.

\section{"Ia Malu Model"}

The findings of my research are the four phases that constitutes the "Ia Malu Model". The model simply offers a path that illustrates how the participants experienced their transition to insulin therapy. This in turn answers the research question. The four phases of the model are: grief, realisation, hopefulness and wholeness. The two fundamental concepts; knowledge transfer and knowledge synthesis, enabled the participants to transition from one phase to another through the relative encounters.

\section{Phase 1: $\quad$ Grief}

\section{(Fa'anoanoaga, Lotomomomo)}

Extensive studies have been conducted on the concept of grief going as far back as 1915 with Freud's acknowledgement, in which he described grief as the process of energy withdrawal from a person that is lost (as cited in Moules, 1998). The work of Kübler-Ross (1969) pioneered methods in the support and counselling of personal trauma, grief and grieving associated with death and dying. This work considerably improved the 
understanding and practices in relation to bereavement. What is special about this work is its transferability outside of death and bereavement, onto understanding personal change and emotional upset resulting from factors other than death and dying (Chapman, 2006). Her work was landmark to considering grief as a "fluid, nonsequential process through the stages originating in shock and denial and resulting in eventual resolution" (Moules, 1998, p. 147).

My research tells us that grief is the first phase of the model to encompass the emotional unrest, suffering and overwhelming experiences of the participants as triggered by the transfer of knowledge. Their feelings of fear, denial, disbelief and refusal arose when they were consulted about having developed diabetes, as well as at the time when discussions began about the need to transition to insulin. The grief that surrounded their adjustments to diabetes was incredible, similar to the findings in the study by Kelly et al. (2005), where the participants saw the outcomes of their struggles with specific impacts as leading to adaptations and change in their lives. The worlds of the person with diabetes and HCP are different. From a diagnosis point of view, the HCP's perspective is the solution to a medical puzzle, while the patient's perspective, diabetes is the starting point for an altered life situation (Hörnstein, Sandström \& Lundman, 2004). The complexities of living with diabetes can generate stumbling blocks, which can take years to overcome for individuals with it (Pouwer \& Hermanns, 2009). It was awfully difficult for the participants to modify their lifestyle and change the food they grew up with to another that was not familiar to them. The participants mostly elaborated on having predominantly negative feelings towards these changes in their lives because of diabetes, and how much it has impacted on their daily functioning. Transitioning to insulin was overwhelming, and it caused them much distress and they refused to contemplate using it. Most of the participants chose to put up with feeling unwell while on oral medications, as long as insulin was not commenced. One participant associated insulin with death, and it being the last hope. The grief that surrounded these aspects in the participant's journey was mind blowing. It is an experience within the phenomenon of loss which can take the forms of another through death, or losses of hope, relationships, job, health, safety, self and so forth (Moules, 1998). Drawing back onto Kübler-Ross' (1969) work, again while it focused on death and 
bereavement, the grief cycle is a useful perspective for understanding our own and other people's emotional reactions to personal trauma and change, regardless of cause. As she described in stage one; denial is a "healthy way of dealing with the uncomfortable and painful situation patients have to live with for a long time” (p. 35). Grief as it seems, is also known in other terms to be chronic sorrow (Lichtenstein, Laska \& Jeffery, 2002) or complicated grief (Love, 2007).

The concept of chronic sorrow had been originally used to describe the profound sadness in parents whose children were mentally impaired and were mourning the loss of the "whole" child. The term chronic sorrow has since expanded to include chronic depression resulting from ill health, long term disability or impairment. One study that examined chronic sorrow in the HIV-positive patients (Lichtenstein et al., 2002) found that although the term meant different things to the men and women that participated, it suggested that chronic sorrow occurs in people with HIV/AIDS. The concept of uncomplicated grief is also referred to as prolonged grief. It describes a general pattern of grief experience whereby, people initially would react with shock or denial to news of loss. People can report feeling numbness and disbelief exhibiting a phase of acute anguish that might emerge. Often complicated grief causing significant functional impairment is assumed to last for about six months (Love, 2007).

Despite the evolving and developing descriptions and emergence of grief, it remains an unavoidable human experience with highly varied features and patterns which "becomes a lifelong and life-changing experience" (Moules, 1998, p. 143). As a result of the participant's grief, three of the four participants stopped taking medications and were hesitant about making changes to their lifestyles. This in turn eventually led to symptomatic presentations such as lethargy, tiredness and infection resulting in admission to hospital. This phase triggered the creation of knowledge and evaluation of their wellbeing and assisted their realisation.

Grief is similar to that of the first layer of thatches on the roof of the fale starting from the eaves. This is significant as it represents the participants living with diabetes as well as the 
beginning of their journey with their use of insulin. Like the thatches being tied one at a time and overlapping one another until that layer is completed before another layer begins, is similar to that of the participants. Little by little, their knowledge of their diabetes and use of insulin will grow over time through means of knowledge transfer and synthesis. The processes of knowledge transfer and synthesis, enabled the participants to transition to the second phase of the Ia Malu model, being realisation.

\section{Phase 2: $\quad$ Realisation}

\section{(Iloatino, Lu'itauina, Talitonuga)}

During the process of knowledge synthesis came the generation of "realisation" or turning points among the participants. This phase refers to the participant's reassessment of their health and realising the best alternative choices for their lives as they anticipate the future alongside their families. Adjustment to disability shows how change occurs as a turning point following psychological reinterpretation of events (Rönkä, Oravala \& Pulkkinen, 2002). Insulin is an extremely effective glucose lowering treatment that is required for type 2 when the pancreas function fails (UKPDS, 1998). One notable phenomenon among the participants was realising the usefulness of insulin in maintaining their wellness and wellbeing. It was noticeable in their narratives that the more they understood insulin, and became aware of its clinical benefits on their health, the more accepting they became in using it. Kübler-Ross (1969) elicited on the fifth stage: acceptance, as "almost void of feelings....as if the pain had gone, the struggle is over" (p. 100). She also maintained that when more time is given to patients alongside some help, will enable them to work through their grief. Although her ideas centred on death and bereavement, the model helps us understand personal trauma and reactions outside death. It is clear that the experiences of the participants in my study have in many ways manifested similar reactions Kübler-Ross (1969) referred to.

Being given time for the patients to adjust and familiarise with insulin can leave positive impression on them. One of the participants affirmed the positive effects of insulin on her to reflect her feelings of "wellness". All participants got familiar with insulin which helped improve their confidence on using it, and they spoke out about feeling well. The 
participants also grasped that if they were to miss their injections, they would retrieve back to feeling lethargic, tired and unwell. This is comparable to the findings reported by Grant, Devita, Singer and Meigs (2003) that patients may be more likely to adhere to their treatment if they believe it alleviates their diabetes symptoms. All the participants shared this common realisation that their lives depend on insulin. They acknowledged that the efforts invested in their health now ensures better outlook in the future for them and especially to live longer for their children and grandchildren. Insulin as they experienced embodies "hope".

The continuous adding of thatches represents the growing awareness of the patient of their health and wellbeing, while appreciating the benefits of insulin. The patient needs to carry on building the fale by thatching it. Although the effort it requires is demanding and tiring, soon enough, the roof of the fale will be completed when it reaches the top. Through the concepts of knowledge transfer and synthesis, the participants are moved to the third phase of their journey. This phase is hopefulness.

\section{Phase 3: $\quad$ Hopefulness}

\section{(Fa'amoemoega, Talotaloga, Fa'atuatuaga)}

"Hope is considered the universal human phenomenon that is essential for overcoming adversity and fostering resilience "(Leung, Silvius, Pimlott, Dalziel \& Drummond, 2009, p. 348). This phase describes the hope that was constantly present among the participants, no matter the hardship. Their revelations emphasised insulin to represent their "hope" and the hopes of their aiga. Using insulin has changed the way they look at life today, after all the acute admissions they've encountered and endured because of poor diabetes management. Since their incredible decision to use insulin, their grief (that originally surrounded their transition to insulin) has eventually faded and their beliefs of insulin have transformed dramatically. This transformation surfaced, because they have been reassured through their own lived experiences with diabetes, that it is manageable and achievable with consistent administration of treatments. This awareness had built on their hopes as they carry on living towards the future. Kübler-Ross (1969) claimed that despite the different stages people go through when confronting tragic news, the one thing that usually persists 
through out is hope. Patients who adopt sensitive measures and logical coping mechanisms in the context of general optimism and hopefulness in diabetes should be more reactive and adherent to daily management (Dunn et al., 1990).

Enhanced feelings of hopefulness emerged for the participants having undergone a massive leap of realisation. The concepts of knowledge transfer and knowledge synthesis helped transitioned them between these phases. Their hopefulness strengthened as they continued using insulin, observed improved glucose readings and subsequent palpable feelings of restored health and wellness. These essential elements give rise to the notion "seeing is believing" which assisted the building of hopefulness among the participants. They became more familiar and were more accepting when they observed improved glucose readings. Insulin as they maintained meant "hope".

The persistence in laying and tying of the thatches on the roof until completion comes with such delight and admiration because the job has not been easy. It requires long hours of hard work and skills. Being able to thatch the entire roof well and securely means it will keep the rain out, and it will be malu (sheltered). The next phase in the participant's journey focuses on wholeness, resulting from their persistence and perseverance to keep going on in life despite living with Type 2 diabetes.

\section{Phase 4: $\quad$ Wholeness}

\section{('Atoaga, 'Ato'atoa)}

This phase refers to the participants' feelings of complete wellness. Although the start of the journey was erratic because of physical suffering and the many uncertainties and doubts that hindered their transition to insulin, the end has been rather rewarding. Their restored health and wellbeing are a testimony to their use of insulin. As they continued taking it, their wellness is maintained and enhanced.

Wholeness reflects the participants sitting at the very top of the fale with feelings of total wellness and total control of their wellbeing. When sitting at the very top, you are able to look down and see everything. For the participants, with insulin on their side, not only they 
feel so much better, but have become conscious of its usefulness to their health and wellbeing. They have experienced the benefits of insulin in their journey which allowed space for adjusting to, and acceptance of insulin.

\section{The concept of knowledge transfer}

The concept of knowledge transfer enabled the participants to process their various experiences. The concept relates to the transfer of knowledge between the doctors or the diabetes nurses or practices and the participants, about diabetes management and the need to transition to insulin to achieve that. The participants were informed about the use of insulin and why it would be the next best option to consider for diabetes management. On the contrary, there was also a transfer of false knowledge between the participants and their family members and friends who provided them with misleading information about insulin and why they should not be taking it (i.e.) what it could potentially do to them if they begin taking it (Bogatean \& Hâncu, 2004; Khan, et al, 2008;). What would you do with all this contradictory information? It made their transition to insulin that much harder.

\section{The concept of knowledge synthesis}

In this case, the concept of synthesis is about processing and synthesising of the knowledge and awareness demanded of the participant's illness. A few factors occurred for the participants, ranging from their assimilation of the knowledge and information communicated to them by their $\mathrm{HCP}$, to acknowledging that their acute suffering was caused by poor diabetes management. The participants noticed that since they used insulin, they were beginning to feel better. Through their wellness came a better awareness of the reasons and causes as to why they felt unwell in the first instance. For example: "if you know your sugar levels are too high then you know obviously you are doing something wrong" (Sione, p. 4). This quote encapsulated the sort of knowledge creation that took place for the participants. This synthesis of knowledge offered room and space for positivity and optimism for the participants which also enabled them to cope with the transition to insulin. Coping mechanisms and strategies would have played an encouraging role in easing the process and so by incorporating an illness into a reconstituted sense of self can be eased by social support, in particular from families (Hörnstein et al, 2004). 
Nurses and health care professionals play a critical role in improving management for people with Type 2 diabetes. Implications for nurses and health care professionals will be discussed in the next following section. But first, is the illustration of the Ia Malu Model as described (See Figure 2). 


\section{Ia Malu Model}

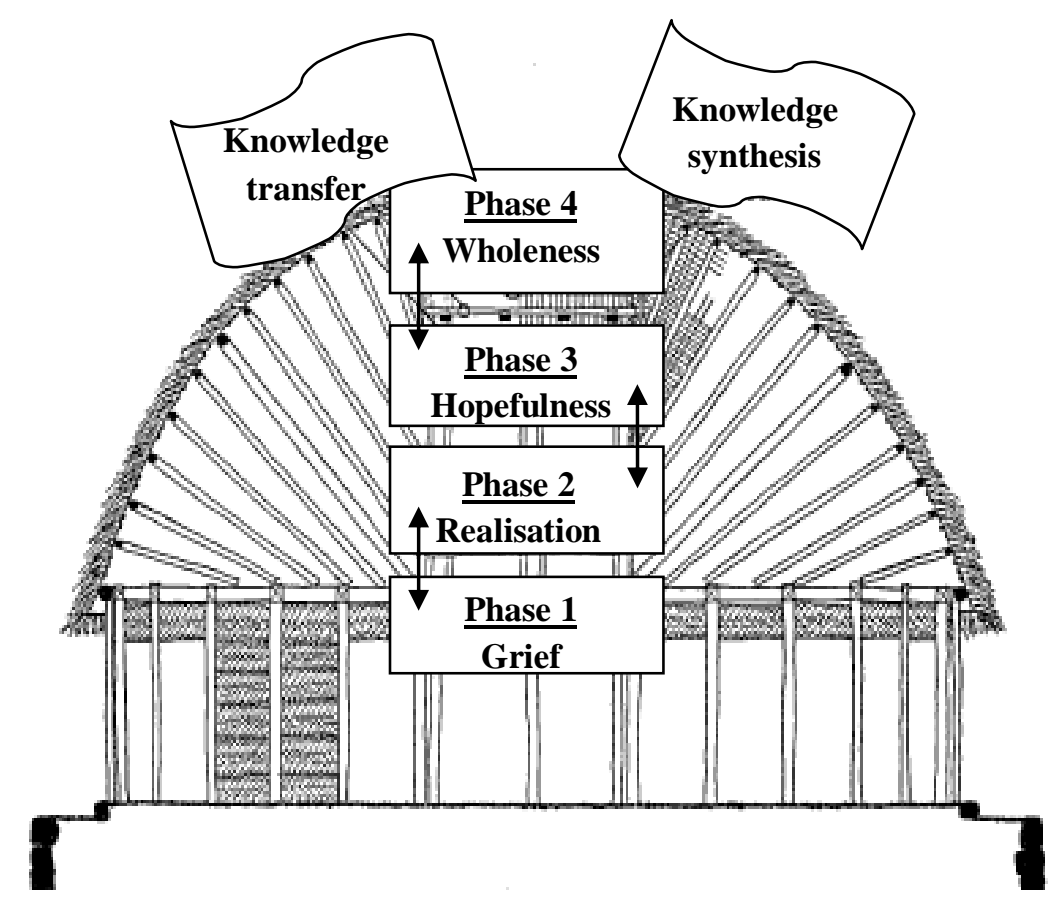

(Picture of fale tele used with permission from UNESCO, Apia, Samoa (1992))

Phase 1: Grief

Phase 2: Realisation

Phase 3: Hopefulness

Phase 4: Wholeness
(Fa'anoanoaga, Lotomomomo)

(Lu'itauina, Iloatino)

(Fa'amoemoega, Fa'atuatuaga)

('Atoaga, 'Ato'atoa)

The processes of knowledge transfer_and knowledge synthesis enabled the participants to transition from one phase to another. 


\section{Implications for Health care professionals}

The building process for a fale is complicated (UNESCO, 1992). The process of coming to terms with a life long condition like diabetes is also complicated for people who are diagnosed with it. Additional demands such as transitioning to insulin increases the complexities in the management of diabetes. When people just get diagnosed with diabetes, they, at that point, are just beginning their life long journey with it. They are required to adjust to lifetime demands, while at the same time adapt to the many stresses imposed on their lives amongst other factors (Dunn et al., 1990). Even if discussing insulin, this process can exhaust the patient because all these will be competing with other interests in their lives. This in turn is the same as starting to build the fale. Building a fale takes a long time and it involves immense hard work. People with diabetes have the tendency to move back and forth within the various phases whether that be assimilating knowledge, grieving, making adjustments or building on hope that helps them get through daily living (Kübler-Ross, 1969). What I mean by back and forth is the countless attempts by the participants to make adjustments, striving to live a normal life whilst processing knowledge and information about managing their diabetes; all these are most likely to take place while feeling unwell most of the time.

It is crucial for nurses/HCP to remember that when sitting down with a patient who is newly diagnosed with diabetes, or about to discuss insulin as a next treatment option, to understand that they are just embarking on a life long journey. Patients at this point are still grieving and this usually lasts for at least the first six months, when their grief is complicated (Love, 2007; Moules, 1998). Patients are realising that developing diabetes or starting insulin is the starting point for an altered life situation (Hörnsten et al., 2004). We do need to be careful that their coming to terms is going to be a long process and it won't

happen overnight. Patients need to build the fale themselves and they are capable of doing this with the help and support of the nurses/HCP.

Like the cagelike framing for the roof, the nurses/HCP must be caring and responsive to the needs of the patients. We need to be culturally sensitive and skilled when delivering health education that supports and encourage diabetes management, in particular when 
working with patients of minority groups. The way nurses deliver care can impact on the patients, so it is exceptionally important for nurses to know how to approach people in the right way (Ramsden, 1994). When nurses understand that they are cultural bearers, they can then work with people from any culture. Benner (1984) emphasised that the more significant learning lies in coping with illness and mobilising recovery, whereby nurses offer information, ways of being, ways of coping and even new possibilities.

In my work as a diabetes nurse within the Pacific community, I realised that I may have at times raced ahead of the patients, and not allow enough time for the patients to process all this overwhelming information. Getting them on to insulin is far more complex than I imagined, and we've got no idea on how patients can live and be happy in their families and communities, while struggling with a chronic illness like diabetes. They struggled to be skilful at giving insulin while at the same time adjust to being diabetic. I now realise that it is exceptionally essential that we remain optimistic and be giving the patients sufficient time to come to terms with not only their diabetes but using insulin as well. People are also labelled as being non-compliant, yet, what would assist their coming to terms would be sufficient time for them to make connections on the efficacy of insulin to their feeling of wellness and restoring of health again. This study has also reminded me to pay special attention to the way I communicate to the people about their diabetes, and be thoughtful of their grief when discussing insulin therapy. Perhaps consider running community based education groups to help change the understanding of the role and need for insulin and to include good role models to share their successful stories with insulin. The need to make available posters and pamphlets in the Samoan language to explain diabetes trajectory and why insulin has a role in it should be considered seriously to resonate awareness and understanding. Nurses/HCPs working with Samoan patients with Type 2 diabetes would need to be informed about the transition issues and highlight strategies that will be helpful to improve the uptake of insulin in this vulnerable group. It is also critical for dissemination of this work that I return to the Samoan radio to share this message to benefit the wider community. 
We have only just begun to discover and unpack, what really happens to Samoan people of their transition to insulin therapy. What will assist in this case is invite patients to communicate specifically what barriers mean to them (Funnell, 2007), rather than offering opposing views. It's fundamental that by walking alongside the patients with diabetes will help get them through their struggles, in this case, reach the very top of the fale, having fulfilled their sense of wholeness. This research acknowledges that it has limitations, which will be discussed next.

\section{Limitations}

A noted limitation of the study concerns the small sample size of only 4 participants, considering, that the sample group was homogenous. A sample size of 10 participants would have been more adequate in a study like this one, to attain maximum variation of the phenomenon studied. Inadequate sample sizes also undermine credibility of the findings. But in spite of this, sampling in qualitative research is not central on numbers, but the very kinds of purposeful sampling that is case oriented and information rich cases (Sandelowski, 1995) critical in generating knowledge and raising awareness on the phenomenon understudied.

This work is the first of its kind in terms of research knowledge on how Samoan people come to terms with their transition to insulin therapy, to form basis for future research in the area of Pacific health and chronic illness. The next section offers suggestions for future research.

\section{Future research}

Diabetes will continue to affect many more Samoan people in the future; therefore, effective measures need to be understood and reinforced in addressing diabetes and related health problems. A beginning point for future research is to test this model in a bigger research sample to check validity and feasibility for the Samoan community. Other research ideas may include testing the model among Samoan people in NZ or between the various Pacific communities. Another would be a comparison study to test for similarities or differences between Samoan people living in NZ and Samoan people in Samoa. 
This study did not include questions on the spiritual beliefs of the participants. Given that Samoa is a deeply religious country, it would be interesting to see how the spiritual wellbeing of Samoan people impact or not on their health beliefs may offer very different perspectives compared to the current themes. These results will provide valid and meaningful conclusions valuable to the health sector and policy makers to draw on, for better use of targeted resources. The conclusion which follows next summarises the contribution offered by this research to the discipline and concludes the thesis.

\section{Conclusion}

As a result of the generosity of the four participants we are just beginning to find out what it is like for Samoan people with Type 2 diabetes, to transition to insulin therapy for improved glycaemic control. Through the remarkable journeys of the participants and the stories of their suffering and adjustments, have become the building blocks to the roof of the fale. Nurses as cultural bearer and healers of health, play a prominent role in building of the fale, by beginning to acknowledge the cultural differences and meaning that vulnerable communities appreciate, as well as their sensitivities around the concepts of knowledge transfer and knowledge synthesis. This research offers a platform for future studies concerning the health of Pacific people and chronic illness. 


\section{Reference List}

Anae, M. M. (2009). Samoans - History and migration. Retrieved March 31, 2010, from http://www.TeAra.govt.nz/en/samoans/1.

Barnes, L., Moss-Morris, R., \& Kaufusi, M. (2004). Illness beliefs and adherence in diabetes mellitus: a comparison between Tongan and European patients. The New Zealand Medical Journal, 117(1188). Retrieved on February 12, 2010, form the New Zealand Medical Association database on http://www.nzma.org.nz/journal

Benner, P. (1984). From novice to experts. California: Addison-Wesley Publishing Co.

Bogatean, M.P., \& Hâncu, N. (2004). People with type 2 diabetes facing the reality of starting insulin therapy: factors involved in psychological insulin resistance. Practice Diabetes International, 21(7), 247 - 252.

Blakely, T., \& Dew, K. (2004). Ethnicity, acculturation and health: who's to judge? The New Zealand Medical Journal, 117(1188). Retrieved on November 14, 2010, from New Zealand Medical Associatio database on http://www.nzma.org.nz/journal

Brown, K., Avis, M., \& Hubbard, M. (2007). Health beliefs of African - Caribbean people with type 2 diabetes: a qualitative study. British Journal of General Practice, 57, $461-469$.

Campos, C. (2007). Addressing cultural barriers to the successful use of insulin in Hispanics with type 2 diabetes. Southern Medical Association, 100(8), 812 - 820.

Chapman, A. (2006). Elizabeth Kübler-Ross - Five Stages of grief. Retrieved March 29, 2010

from http://www.businessballs.com/elizabeth kubler ross five stages of grief.htm 
Cartwright, A., Woodward, A., \& Wallymahmed, M. (2006). Introduction of a care pathway for people with type 2 diabetes starting on insulin. Journal of Diabetes Nursing, $10(1), 14-18$.

Corral, I., \& Landrine, H. (2008). Acculturation and Ethnic-Minority health behaviour: A test of the Operant Model. Health Psychology, 27(6), 737 - 745.

Culhane-Pera, K., Her, C., \& Her, B. (2007). We are out of balance here: A Hmong Cultural model of diabetes. Journal of Immigrant Minority Health, 9, 179 - 190.

Davies, K. (2006). Addressing the needs of an ethnic minority diabetic population. British Journal of Nursing, 15(9), 516 - 519.

Diabetes Control and Complications Trial Research Group. (1993). The Effect of intensive treatment of diabetes on the development of long-term complications in insulindependent diabetes mellitus. New England Journal of Medicine, 329, 977 - 986.

Diabetes New Zealand. (2006). Diabetes Fact Sheet. Retrieved September 29, 2007, from http://www.diabetes.org.nz/resources/DiabetesFacts2006.pdf.

Dingley, C., \& Roux, G. (2003). Inner strength in Older Hispanic Women with chronic Illness. Journal of Cultural Diversity, 10(1), $11-22$.

Dunn, S. M., Beeney, L. J., Hoskins, P. L. \& Turtle, J. R. (1990). Knowledge and attitude change as predictors of metabolic improvement in diabetes education. Social Science Medicine, 31(10), 1135 - 1141.

Durie, M. (1998). Whaiora: Maori Health Development (2 ${ }^{\text {nd }}$ Edition). Oxford University Press. 
Eldor, R., Stern, E., Milicevic, Z., \& Raz, I. (2005). Early use of insulin in type 2 diabetes. Diabetes Research and Clinical Practice, 68S1, S30 - S35.

Fairbairn-Dunlop, P., \& Makisi, G. S. (2003). Making our place: growing up PI in New Zealand. Palmerston North: Dunmore.

Foliaki, S., \& Pearce, N. (2003). Prevention and control of diabetes in Pacific people. British Medical Journal, 327, 437 - 439.

Funnell, M. (2007). Quality of Life and Insulin Therapy in Type 2 Diabetes Mellitus. Insulin, 3(1), $31-36$.

Glanz, K., Rimer, B. K., \& Lewis, F. M. (2002). Health behaviour and health education, theory research and practice. San Francisco: Wiley \& Sons.

Grant, R. W., Devita, N. G., Singer, D. E., \& Meigs, J. B. (2003). Improving adherence and reducing medication discrepancies in patients with diabetes. Annual Pharmacother, 37, $962-969$.

Greenhalgh, T., Helman, C., \& Chowdhury, A.M. (1998). Health beliefs and folk models of diabetes in British Bangladeshis: A qualitative study. British Medical Journal, $316,978-983$.

Grunberger, G. (2009). The importance of early insulin adoption in type 2 diabetes management. The Internet Journal of Family Practice, 7(2), 1 - 28.

Hansen, E. C. (2006). Successful Qualitative Health Research: A practical introduction. Australia: Allen \& Unwin.

Health Research Council. (2004). The Health Research Council of New Zealand: Guidelines on Pacific Health Research. Auckland, New Zealand, Author. 
Hörnstein, Å., Sandström, H., \& Lundman, B. (2004). Personal understanding of illness among people with type 2 diabetes. Journal of Advance Nursing. 47(2), $174-182$.

Kelly, G., Lawrence, J. A., \& Dodds, A. E. (2005). Women's developmental experiences of living with Type 1 diabetes. Qualitative Social Research, 6(1). Retrieved on December 2, 2009, from the Forum Qualitative Social Research database on http://www.qualitative-research.net/fqs

Khan, H., Lasker, S. S., \& Chowdhury, T. A. (2008). Prevalence and reasons for insulin refusal in Bangladeshi patients with poorly controlled Type 2 diabees in East London. Diabetic Medicine, 25, 1108 - 1111.

King, A. (2000). The New Zealand Health Strategy. Wellington: Ministry of Health.

King, A. (2001). The Pacific Health Strategy. Wellington: Ministry of Health.

Krämer, A. (1903). Die Samoa-Inseln. Germany: Stuttgart.

Kübler-Ross, E. (1969). On death and dying: What the dying have to teach doctors, nurses, clergy, and their own families. Macmillan Publishing Co: New York.

Lay, G. (2000). Samoa: Pacific Pride. Pasifika Press Ltd: Auckland, New Zealand.

Leung, K. K., Silvius, J. L., Pimlott, N., Dalziel, W., \& Drummond, N. (2009). Why health expectations and hopes are different: the development of a conceptual model. Health Expectations, 12, 347 - 360.

Lichtenstein, B., Laska, M. K., \& Jeffrey, M. C. (2002). Chronic sorrow in the HIVPositive patient: Issues of race, gender and social support. AIDS Patient Care and STDs, 16(1), $27-38$. 
Love, A. W. (2007). Progress in understanding grief, complicated grief and caring for the bereaved. Contemporary Nurse, 27(1). $73-83$.

Lupton, D. (1995). Perspectives on power, communication and the medical encounter: implication for nursing theory and practice. Nursing Inquiry, $157-263$.

Mathews, S. M., Peden, A. R., \& Rowles, G. D. (2009). Patient - provider communication: Understanding diabetes management among adult females. Patient Education and Counselling, 76, $31-37$.

Meleisea, M. (1987). Lagaga: A short history of Western Samoa. University of the South Pacific, Suva, Fiji.

Minichiello, V., Aroni, R., \& Hays, T. (2008). In-Depth Interviewing ( $3^{\text {rd }}$ Edition).Sydney, Australia: Pearson Education Australia.

Ministry of Health. (2002). Modelling diabetes: a summary. Wellington: Author.

Ministry of Health. (2005). Ministry of Health: Statement of Intent for 2006 - 2009. Wellington: Author.

Ministry of Health. (2008). Improving Quality of care for Pacific Peoples. Wellington: Author.

Ministry of Health. (2010). 'Ala Moui: Pathways to Pacific Health and Wellbeing 2010 2014. Wellington: Author.

Moata'ane, L. M., Muimui-Heata, S., \& Guthrie, B. (1996). Tongan perceptions of diet and diabetes mellitus. Journal of the New Zealand Dietetic Association, 50(2), 52 - 56. 
Moules, N. (1998). Legitimising Grief: Challenging beliefs that constrain. Journal of Family Nursing, 4(2), $142-166$.

Nakar, S., Yitzhaki, G., Rosenberg, R., \& Vinker, S. (2007). Transition to insulin in Type 2 diabetes: family physicians' misconception of patients' fears contributes to existing barriers. Journal of Diabetes and Its Complications, 21, 220 - 226.

Nathan, D.M. (2007). Finding new treatments for diabetes - how many, how fast....how good? The New England Journal of Medicine, 356(5), 437 - 440.

New Zealand Guideline Group. (2003). Evidence-based best practice guideline: Management of Type 2 diabetes. Wellington: New Zealand Guideline Group.

New Zealand Herald. (2007, February 21). Pacific Islands 'worst in world for obesity'. The New Zealand Herald. Retrieved February $2^{\text {nd }}, 2007$, from http://www.nzherald.co.nz/nz/news/article.cfm

Pacific Islands 'worst in world for obesity'. (2007, Feb 21). The New Zealand Herald. Retrieved February 27, 2007, from http://www.nzherald.co.nz/nz/news/article

Peyrot, M., Rubin, R. R., Lauritzen, T., Skovlund, S. E., Snoek, F. J., Matthews, D. R., Landgraf, R. L., \& Kleinebreil, L. (2005). Resistance to insulin therapy among patients and providers: results of the cross-sectional Diabetes Attitudes, Wishes and Needs (DAWN) study. Diabetes Care, 28, 2673 - 2679.

Pitt, D., \& Cluny, M. (1974). Emerging pluralism: The Samoan community in New Zealand. Auckland: Longman Paul.

Porth, C. M ., \& Matfin, G. (2009). Pathophysiology - Concepts of altered health states ( $8^{\text {th }}$ Edition). Lippincott: Williams \& Wilkins. 
Pouwer, F., \& Hermanns, N. (2009). Insulin therapy and quality of life. A review. Diabetes/Metabolic Research Review, 25(Suppl 1): s4 - S10.

Pulotu - Endermann, F. K. (2001). Fonofale Model of Health. Unpublished Model of Health.

Ramsden, I. (1994). Accepting others for what they are. New Zealand Quality Health Newsletter of the New Zealand Council on Healthcare Standards, 11, $10-11$.

Robinson, T., Simmons, D., Scott, D., Howard, E., Pickering, K., Cutfield, R., Baker, J., Patel, A., Wellingham, J., \& Morton, S. (2006). Ethnic difference in Type 2 diabetes care and outcomes in Auckland: a multiethnic community in New Zealand. The New Zealand Medical Journal, 119(1235), 1997 - 2007.

Rönkä, A., Oravala, S., \& Pulkkinen, L. (2002). "I met my wife of mine and things got onto a better track": Turning points in risk development. Journal of Adolescence, $25,47-63$.

Sandelowski, M. (1994). We are the stories we tell: Narrative knowing in nursing practice. Journal of Holistic Nursing, 12(1), 23 - 33.

Sandelowski, M. (1995). Sample size in Qualitative Research. Research in Nursing, 18, $179-183$.

Sandelowski, M. (2000). Focus on research methods. Whatever happened to qualitative description? Research in Nursing and Health, 23, 334 - 340.

Sandelowski, M. (2004). Using Qualitative Research. Quality Health Research, 14, 1366 1386.

Schütz, A. (1962). The problem of social reality. Martinnus Nijhoff: The Hague. 
Simmons, D. (1999). Diabetes in Maori and Pacific Islands people. New Ethicals Journal, Feb, $45-49$.

Simmons, D., Voyle, J. A., Fou, F., Feo, S. \& Leakehe, L. (2004). Tale of two churches: differential impact of a church-based diabetes control programme among Pacific Islands people in New Zealand. Diabetic Medicine, 21, $122-128$.

Stair, J. B. (1983). Old Samoa or Flotsam and Jetsam from the Pacific Ocean. Papakura, New Zealand: R. McMillan Publisher.

Statistics New Zealand. (2006). Census 2006. Wellington, NZ: Author.

Statistics New Zealand. (1991). Census 1991. Wellington NZ: Author.

Steyne, N., Mann, J., Bennett, P., Temple, N., Simmet, P., \& Tuomilehto, J., Lindström, J., \& Louheranta, A. (2003). Diet, nutrition and the prevention of type 2 diabetes. Public Health Nutrition, 7(1A), 147 - 165.

Swinburn, B., Amosa, H., \& Bell, C. (1998). The Ola Fa'autauta project: The process of developing a church-based health programme. Pacific Health Dialog, 4(2), 20 25.

Taylor, B., Kermode, S., \& Roberts, K. (2006). Research in Nursing and Health care: Evidence for practice ( $3^{\text {rd }}$ Edition). Nelson, Australia: Thomson.

Trochim, W. M. K., \& Donnelly, J. P. (2007). The Research Methods Knowledge Base (3 ${ }^{\text {rd }}$ Edition). Mason OH: Thomson Publishing.

Tukuitonga, C., \& Finau, S. A. (1997). The health of Pacific peoples in New Zealand up to the early 1990's. Pacific Health Dialog, 4(2), 59 -66. 
Turner, G. (1984). Samoa: Hundred years ago and long before. Apia, Samoa: Commercial Printers Ltd.

UK Prospective Diabetes Study (UKPDS) Group. (1998). Intensive blood-glucose control with sulphonylureas or insulin compared with conventional treatment and risk of complications in patient with type 2 diabetes (UKPDS 33). Lancet, 352, 837 853.

UNESCO. (1992). The Samoan Fale. Bangkok, Thailand: Author.

Vaioleti, T. M. (2006). Talanoa research methodology: A developed position on Pacific Research. Waikato Journal of Education, 12.

Walsh, M. E., Katz, M. A., \& Sechrest, L. (2002). Unpacking cultural factors in adaptation to type 2 diabetes mellitus. Medical Care, 40(1), Supplement, 129 - 139.

Wang, C., Abbott, L., Goodbody, A. K., Wait-Ting, Y. H., \& Rausch, C. (1999). Development of a community-based diabetes management program for Pacific Islanders. The Diabetes Educator, 25, 738 - 746.

Weinger, K., \& Beverley, E. A. (2010). Barriers to achieving glycaemic targets: Who omits insulin and why? Diabetes Care, 33(2), 450 - 452.

Williams, G., \& Pickup, J. (2004). Textbook of diabetes (Vol. 2). Oxford, UK: Blackwell Science Ltd.

Wong, G. (2002, October). Pride of the Pacific. Metro, 256, 106 - 107.

World Health Organisation. (2010). Defining Diabetes. Retrieved November 27, 2010, from http://www.who.int/diabetesactiononline/diabetes/en/ 


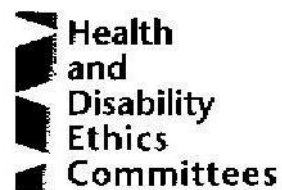

27 June 2008

Ms Sera Tapu - Ta'ala

10 The Yardarm

Whitby

Wellington 5024

Dear Sera

\author{
Central Regional Ethics Committee \\ Ministy of Health \\ Level 2, 1-3 The Terrace \\ POBOx 5013 \\ Wellington \\ Phone (04) 496 2405 \\ Fax (194) 4962191
}

How do Samoan people with Type 2 diabetes mellitus who need insulin for good glycaemic control, come to terms with their therapy 7 qualitative exploratory descriptive study.

Ms Sera Tapu - Ta'ala

Hutt Valley DHB

CENI08/051025

The above study has been given ethical approval by the Central Regional Ethics Committee. A list of members of this committee is attached.

Approved Documente

- Participant Information sheet and Consent Form Version 2, dated 9 June 2008

- Fa'amatalaga au'ili'ili'ili I le sa' ili' iliga Information Sheet and Consent Form Version 1, dated 9 June 2008 Samoan.

- Interview outline and questions Version 2, dated 9 June 2008

- Tran scriber statement of confidentiality Version 2, dated 9 June 2008

- Letter to Health Care Professionals Version 2, dated 9 June 2008

\section{Certification}

The Committee is satisfied that this study is not being conducted principally for the benefit of the manufacturer or distributor of the medicine or item in respect of which the trial is being carried out.

\section{Accreditation}

The Commiltee involved in the approwal of this study is accredited by the Health Research Council and is constituted and operates in accordance with the Operational Standard for Ethics Committees, April 2006.

\section{Final Report}

The study is approved until 31 March 2009. A final report is required at the end of the study. The report form is available on http:/Www.ethicscommittees.health.govt.nz and should be forwarded along with a summary of the results. If the study will not be completed as advised, please forward a progress report and an application for extension of ethical approval one month befcre the above date.

\section{Requirements for SAE Reporting}

The Principal Investigator will inform the Committee as soon as possible of the following

- Any related study in another country that has stopped due to serious or unexpected adverse events

- withdrawal from the market for any reason

- all serious adverse events occurring during the study in New Zealand which result in the investigator breaking the blinding code at the time of the SAE or which result in hospitalisation or death.

- all serious adverse events occurring during the study worldwide which are considered related to the study medicine. Where there is a data safety monitoring board in place, serious adverse events occurring outside New Zealand may be reported quarterly.

All SAE reports must be signed by the Principal Investigator and include a comment on whether helshe considers there are any ethical issues relating to this study continuing due to this adverse event. It is assumed 
by signing the report, the Principal investigator has undertaken to ensure that all New Zealand investigators are made aware of the event.

\section{Amendments}

All amendments to the study must be advlsed to the Committee prior to their implementation, except in the case where immediate implementation is required for reasons of safety. in such cases the Committee must be notified as soon as possible of the change.

As part of the conditions of approving a proposal, committees may require an independent review or audlt of approved research or innovative practice at any time.

Please quote the above ethics committes reference number in all correspondence.

The Principal Investigator is responsible for advising any other study sites of approwals and all other correspondence with the Ethics Committee.

It should be noted that Ethics Committee approval does not imply any resource commitment or adminlstrative facllitation by any healthcare provider within whose facility the research is to be carried out. Where applicable, authority for this must be obtalned separately from the appropriate manager within the organisation.

Yours sincerely

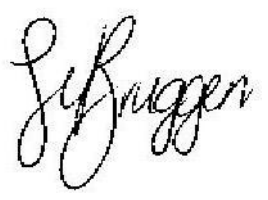

Jlska van Bruggen

Central Regional Ethics Commlttee Adminlstrator

Email: jiska_van_br.uggen@moh.gout.nz 


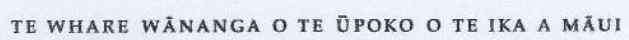

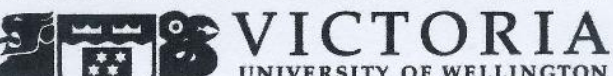 \\ UNIVERSITY OF WELLINGTON}

RESEARCH PARTICIPANTS NEEDED FOR DIABETES RESEARCH

This is a Samoan based study to explore beliefs and perceptions specific to how Samoan people make the transition to insulin therapy and what this means for them.

\section{ARE YOU:}

An Adult Samoan, have Type 2 Diabetes, commenced insulin in the last twelve months?

Do you reside in the Hutt Valley area?

If you are and would be interested in sharing your experiences, stories and thoughts about what being on insulin has meant for you, then I would like to hear from you.

This fully approved study is funded by the Health Research Council.

The study will be conducted by myself, Sera Tapu-Ta'ala as part of my Master of Nursing (Clinical) degree.

I am a Registered Nurse and of Samoan ethnicity. The study is supervised by Assoc. Professor Cheryle Moss Graduate School of Nursing \& Midwifery, Victoria University of Wellington.

For more information, please contact Sera on (04) 2348878 or (mobile ) 0274793025 or email: tuuilasera@student.vuw.ac.nz

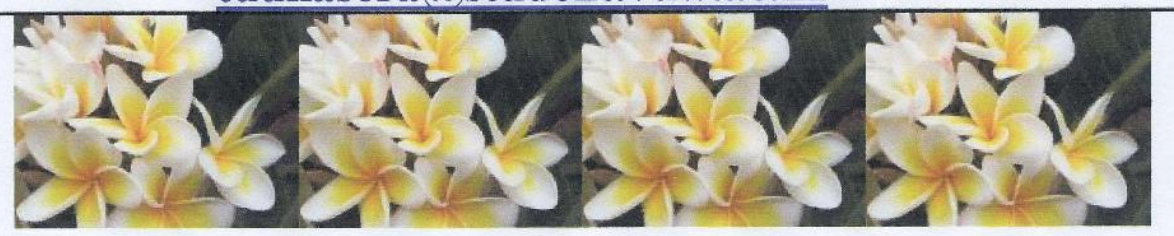




\section{Appendix 3}

$$
\begin{aligned}
& \text { Health } \\
& \text { and } \\
& \text { Disability } \\
& \text { Ethics } \\
& \text { Committees }
\end{aligned}
$$

20 October 2008

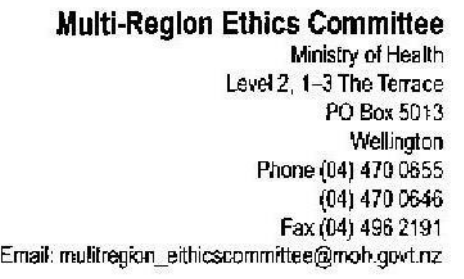

Ms Sera Tapu - Ta'ala

10 The Yardarm

Whitby

Wellington 5024

Dear Sera

CEN/0805/025

How do Samoan people with Type 2 dlabetes mellitus who need insulin for good glycaemic control, come to terms with their therapy? A qualitative exploratory descriptive study.

Ms Sera Tapu - Ta'ala

Hutt Valley DHB

List documents received:

New inclusion criteria for recruiting participants to this study:

- Identified as Samoan

- Age 18 years and over

- Have Type 2 diabetes

- Resides in Hutt Valley DHB

- Commenced insulin in the last 36 months

Thank you for submitting the above amendment, which was considered by the Chairperson urider delegated authority and approved.

\section{Please quate the above ethics committee reference number in all correspondence.}

Yours sincerely

S Scott

Sonia Scott

Central Regional Ethics Commlttee Administrator

Email: sonia_scott@moh.govt.riz 


\section{INFORMATION SHEET}

\section{RESEARCH TITLE:}

How do Samoan people with Type 2 diabetes mellitus that need insulin for good glycaemia control, come to terms with their therapy? A qualitative exploratory descriptive study.

\section{ABOUT THE STUDY:}

Type 2 diabetes is a life-long chronic illness affecting many Pacific people in our communities. Insulin therapy is proven to be the most effective treatment for better control. It is important for diabetes management and control to understand the psychological factors of the Pacific people towards insulin therapy. There is virtually no research involving the Pacific community in relation to what insulin therapy means for them nor processes used or encountered as to how they adjust to insulin therapy.

The purpose of this qualitative study is to explore and describe the beliefs and perceptions of Samoan people (10) with Type 2 diabetes regarding their transition on to insulin therapy. The study is specifically interested in learning from people who have recently commenced or transitioned on to insulin therapy for improved diabetes control in the last twelve months. Their life stories, accounts and experiences will provide insights on how they felt, what they believe about the therapy and what processes of adjusting to the therapy and lifestyle have been for them.

Sera Tapu-Ta'ala, is a Diabetes nurse and of Samoan ethnicity who will be undertaking this study, to complete her Master of Nursing (Clinical) degree. The study will be supervised by Associate Professor Cheryle Moss of the Graduate School of Nursing, Midwifery and Health, Victoria University of Wellington. This study has received ethical approval from the Central Regional Ethics Committee.

Participation for the study is entirely voluntary and will be completely confidential. The participants will not be identified in the findings at all. If you decide to participate in this study to share your experiences, you will be informally interviewed by Sera. The interviews will be recorded and take no longer than one and a half hours. A written copy of your interview will be given to you once it is available. If at any given time you wish to withdraw from the study, you can do so without any negative consequences.

If you have recently commenced insulin therapy (in the last 12 months), are aged over 18 years and would like to participate in this study, the researcher will explain the study to you before you consent. If you are interested in participating or learn more about the study, please contact Sera Tapu-Ta'ala on (04) 2348878 (after hours) or (mobile) 0274793025 or email: tuuilasera@student.vuw.ac.nz 


\section{Appendix 5}

\section{FA'AMATALAGA AU'ILI'ILI I LE SA'ILI'ILIGA (INFORMATION SHEET - SAMOAN VERSION)}

\section{ULUTALA O LE SA'ILI'ILIGA:}

E fa'apefea i tagata Samoa o lo'o aafia i le gasegase o le suka - Ituaiga 2, ona o latou talia le suiga o togafitiga i le tui inisulini auā le va'ava'aia ma le sologa lelei o lona gasegase?

\section{AGAI I LE SA'ILI'ILIGA:}

O le gasegase o le Suka - Ituaiga 2, ua avea ma fa'afitauli tele ma ua fa'atupula'ia i tagata Pasefika. O le vaila'au o le inisulini, ua fa'amaonia lona aoga i le va'ava'aiga lelei o le suka. E fa'atauaina tele i lenei va'ava'aiga o le suka le au'ili'ili o le malamalama'aga i lagona ma mafaufauga o tagata Pasefika, a o gafa ai ma lenei togafitiga. E le o i ai i le taimi nei nisi sa'ili'iliga po'o ni su'esu'ega, o fesiligia ai lagona ma mafaufauga o tagata Pasefika a o gafa ma le fa'atinoina o le tui o le inisulini mo le va'ava'aiga o le gasegase o le suka.

O le naunauta'iga o lenei sa'ili'iliga, o le fia iloa ma fia fa'atalanoa talitonuga ma lagona o ni tagata Samoa (se 10) ua aafia i le gasegase o le suka - Ituaiga 2, a o o latou fa'atinoina le tui o le vaila'au o le inisulini. O le 'autu ina ia maua so'u a'oa'oina mai ia i latou nei, o e na amatalia le fa'atinoina o le tui o le vaila'au o le inisulini i totonu o le 12 masina talu mai. O lā latou fa'asoa mai i o latou lagona ma tomai, o le a avea lea ma molimau ini ō lagona, ni ō latou talitonuga i le tui o le vaila'au o le inisulini, a e pe na fa'apefea ona o latou gafatia lea la'asaga i lo latou olaga.

O Sera Tapu-Ta'ala, o se tamaitai tausimai pasi Samoa o lo'o galue fa'apitoa i 'auaunaga patino i le gasegase o le suka. O ia o le a gafa ma le fa'atinoina o lenei sa'ili'iliga auā le fa'amae'aina o lona fa'ailoga o le Master of Nursing (Clinical). O lenei sa'ili'iliga o le a faia i lalo o le va'ava'aiga a le Polofesa Lagolago mai le Matata a Tausimai Pasi, Fa'atosaga ma le Soifua Maloloina i le Iunivesite a Vitoria i Ueligitone, e suafa ia Dr Cheryle Moss.

E lē fa'amalosia lou auai ma o fa'amaumauga uma o le a malu puipuia lava. E lē fa'ailoaina suafa o i latou e auai i luga o ni tusitusiga po'o ni fa'amaumauga. A tonu i lou manatu e te fia auai e fa'asoa mai ou lagona ma talitonuga, e muamua lā ona oulua talatalanoa ma Sera. O le a pu'eina nei talatalanoaga, ma o le a lē sili atu i le itula ma le afa le umi. E tau'a'aoina atu lau kopi o le lua talatalanoaga pe afai ae uma ona maea tāpena. Afai e i ai se taimi mulimuli ane ua e manatu e te fa'ama'amulu mai i lenei sa'ili' iliga, e mafai lava e aunoa ma sou afaina. 
O lea la afai na amataina lou tui o le inisulini i totonu o le 12 masina, silia ma le 18 tausaga lou soifua ma i ai le naunau e te fia auai i lenei sa' ili'iliga, e muamua ona fa'amanino ma fa'amatala ia te oe le 'auga ma le 'autu. Afai o e fia malamalama atili i lenei sa'ili'iliga, fa'amolemole fa'afeso'otai mai Sera Tapu-Ta'ala i le numera telefoni (04) 2348878 (i le uma o galuega), po'o le telefoni fe'avea'i 0274793025, po’o le i-meli tuuilasera@student.ac.nz 
Appendix 6

TE WHARE WĀNANGA O TE ŪPOKO O TE IKA A MĀUI

\section{CONSENT FORM}

\section{Research Title:}

How do Samoan people with Type 2 diabetes mellitus who need insulin for good glycaemia control, come to terms with their therapy? A qualitative exploratory descriptive study.

I have had the details of the study explained to me by the Researcher. All my questions have been answered to the best of my satisfaction and I have understood the aim, purpose and objectives of the study. Signing this form means that I consent to participate in the study, be interviewed by the researcher and have our interview recorded. I understand that:

- My participation in the research is entirely voluntary.

- I have the right to withdraw from the study and/or decline to answer any questions without negative consequences.

- My identity will be anonymous and pseudonyms will be used in place of my name for transcripts confidentiality and security of data.

- All data will be kept in a secured lock and keyed cupboard, accessible only by the Researcher and Supervisor.

- I have the right to request the raw materials belonging to me at any given time in the study.

- if I have any concerns arising as a result of the Researcher's conduct, I will be able to contact the Central Regional Ethics Committee on telephone number (04) 4962405 .

I, (Name) (Signature)

On this day

Date: 
Fully understands that my participation is voluntary and I consent to participate in this study carried out by Sera Tapu-Ta'ala as part of her Master of Nursing (Clinical) degree at Victoria University of Wellington.

I consent for the transcripts from this research to be re-used for a larger research study in this topic area to be undertaken by Sera Tapu-Ta'ala and her Supervisor Dr Cheryle Moss (please tick which ever applies).

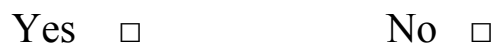

Principal Researcher:

Signature:

Date:

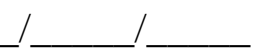




\section{Appendix 7}

TE WHARE WĀNANGA O TE ŪPOKO O TE IKA A MĀUI

\section{LAU MALIEGA MA LE FA'ATANAGA MO LOU 'AUAI (CONSENT FORM - SAMOAN VERSION)}

\section{ULUTALA O LE SA'ILI'ILIGA:}

E fa'apefea i tagata Samoa o lo'o aafia i le gasegase o le suka - Ituaiga 2, ona o latou talia le suiga o togafitiga i le tui inisulini auā le va'ava'aia ma le sologa lelei o lona gasegase?

Ua ia te au fa'amaumauga mo le sa'ili'iliga, ma ua uma foi ona fa'amanino mai e le o le a fa'atinoina. Ua ma'ea ona tali mae'aea' a'u fesili ma ua ou manino foi i le 'auga, autu ma le fa'apogai o lenei sa'ili'iliga. O lo'u sainia o lenei lautusi, e fa'ailoa atu ai ua ou malie atoatoa ou te auai i lenei sa'ili'iliga, ma ua tu'u atu lau fa'atanaga e fa'atalanoa ai au ma ua ou silafia o le a pu'eina lenei talatalanoaga.

\section{Ua ou malamalama e:}

- Lè fa'amalosia lou auai.

- I ai lau aia tatau ou te fa'ama'amulu ai mai lo'u auai ai pea i lenei sa'ili' iliga e aunoa ma so'u afaina.

- Fa'alilolilo ma malu puipuia lou Suafa mai ini ou manatu fa'aalia; o le mafua'aga lea o le a fa'aogaina ai se isi Suafa e ese mai lou Suafa moni.

- Teu malu fa'amaumauga uma e patino i lenei sa'ili'iliga i se kapoti e fai sona loka ma se ki, ma e na o lē lava e gafa ma le sa'ili'iliga fa'atasi ai ma lana Supavaisa/Faiaoga e mafai ona āsia nei fa'amaumauga.

- I ai lau aia tatau oute tāpāina ai au lava fa'amaumauga i so’o se taimi.

- Mafai ona ou feso'otai i le Central Regional Ethics Committee i le telefoni numera (04) 4962405, pe afai e i ai sou fa'agaulemalie po'o so'u popolega i le va ai ma lē o le a gafa ma lenei sailiiliga. 
O au o (Suafa)

La'u Saini

I lenei aso

Aso: 1

Ua ou manino atoatoa e le fa'amalosia lo'u auai, ma ua ou malie ma taliaina lo'u auai i lenei sa'ili'iliga e pei ona gafa ai ma Sera Tapu-Ta'ala, auā le fa'amae'aina o lona fa'ailoga o le Master of Nursing (Clinical) mai le Iunivesite A Vitoria, Ueligitone.

Ua ou malie i le toe fa'aaogaina o a'u fa'aupuga/tusitusiga/fa'amaumuaga maua mai i lenei sa'ili'iliga, auā nisi sa'ili'iliga tetele o afuafua mo le lumanai i luga lava o le mataupu lenei o le a fa'apea ona faatinoina e Sera Tapu-Ta'ala ma lana Faiaoga/Supavaisa o Dr Cheryle Moss (fa'amolemole fa'ailoa mai lau tali).

Ioe $\quad \square \quad$ Leai $\square$

Igoa o lē o le a gafa ma le Sa'ili'iliga

Saini

Aso

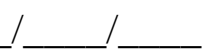


Appendix 8

TE WHARE WĀNANGA O TE ŪPOKO O TE IKA A MĀUI

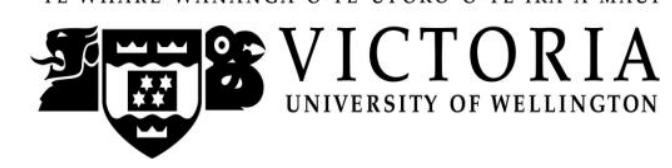

\section{KEY QUESTIONS AND INTERVIEW GUIDE}

\section{During interviews with Participants:}

Initially at the start of each interview, I will engage the participant in a discussion briefly about their diabetes. This will enable us to establish a good lead in to the key questions to contain data generation.

- Introduce Participant (Audiotaped)

- Use confidential labelling to maintain Confidentiality (e.g.) use pseudonym accordingly

- Demographic datasheet to fill out.

- Engage participant in a brief discussion about their diabetes (e.g.) Would you like to tell me a little bit about your diabetes? Has anyone else got diabetes in your family? Do you feel you have sufficient knowledge about diabetes? What do you know about insulin therapy?

\section{Key Questions:}

1. Tell me what it's been like for you since you were diagnosed with diabetes.

2. Could you tell me how you felt at the time you were told about the possibilities of commencing insulin therapy?

3. What were your thoughts and feelings?

4. Were there any factors that got in the way (that delayed) your acceptance of insulin? If so - what did you do to manage these? If not - then what helped your acceptance of insulin?

5. What personal struggles or desires did you and your family confronts in order to come to terms with these transitions?

6. How have you adjusted to this change in your life?

7. Can you tell me how you feel now about using insulin?

8. What advise would you give to the Samoan community out there? 


\section{Samoan translation of key questions:}

1. E mafai ona e ta'u maia le tulaga sa e o'o i ai ina ua iloa ua maua oe i le ma'i suka?

2. O le a se lagona na o'o ifo i lou tagata ina ua fa'ailoa atu e i ai le taimi e ono amata ai sou tui o le inisulini?

3. O a ni mafaufauga po'o ni faalogona na o'o ia te oe?

4. O a ni fa'afitauli na lua fesaga'i na mafua ai ona tuai amata ma taliaina (e lau Susuga/e oe le tina) le tui o le inisulini? Afai na i ai ni mafua'aga, na fa'apefea ona e fa'atautaia lea vaega? A e afai e leai, o a ni auala na fa'afaigofieina ai lou taliaina o le tui?

5. O a ni fete'enaiga/fa'afitauli/vaivaiga fa'aletagata, po'o ni mana'oga o lo'o ia oe aemaise o si tou aiga, ina ia ofi gofie/malie mai pea lenei suiga?

6. O fa'apefea ona fetu'una'i lou soifuaga/lou olaga i le taimi nei pe a fua i le suiga lea ua i ai talu ona fai lou tui?

7. E mafai ona e talatalanoa mai i le tulaga o i ai ou lagona i le taimi nei a o gasolo ma fa'aauau le fa'atinoina o lou tui?

8. O le a se fautuaga e te mana'omia ona momoli atu i tagata Samoa?

E momoli atu le fa'afetai tele ma le agaga maualalo ona o le fa'aavanoaina foi o lou taimi aemaise o le ofoina mai o lou tomai ma lau fa'asoa ma i lou i ai foi ma le fa'atinoina o lou tui aua lava le va'ava'aiga ma le lelei alualu i luma o lou suka.

Faafetai tele lava. 
Appendix 9

\section{TRANSCRIBER'S STATEMENT OF CONFIDENTIALITY}

\section{Research Title:}

How do Samoan people with Type $\mathbf{2}$ diabetes mellitus that need insulin for good glycaemia control, come to terms with their therapy? A qualitative exploratory descriptive study.

I have read the information about the study and have had my questions answered by the researcher to the best of my satisfaction. I fully understand that the transcripts and all other research materials are to be accessed only by the researcher, Sera Tapu-Ta'ala and Supervisor Cheryle Moss.

Most importantly that I keep the information contained in the tapes and transcripts secured while in my care. I agree that I am not to disclose any of its contents to anyone else other than the researchers.

Transcriber's Full Name:

Transcriber's Signature:

Date:

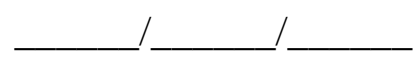

Principal Researcher's signature:

Date:

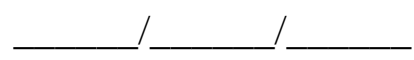

